Prepared for the U.S. Department of Energy

under Contract DE-AC05-76RL01830

\title{
Wide Area Security Region Final Report
}

\author{
YV Makarov \\ $\mathrm{P}$ Du \\ $S$ Lu \\ TB Nguyen \\ $X$ Guo \\ JW Burns \\ JF Gronquist
}

March 2010

\section{Pacific Northwest}

NATIONAL LABORATORY

Proudly Operated by Battelle Since 1965 



\title{
DISCLAIMER
}

This documentation was prepared as an account of work sponsored by an agency of the United States Government. Neither the United States Government nor any agency thereof, nor Battelle Memorial Institute, nor any of their employees, makes any warranty, express or implied, or assumes any legal liability or responsibility for the accuracy, completeness, or usefulness of any information, apparatus, product, or process disclosed, or represents that its use would not infringe privately owned rights. Reference herein to any specific commercial product, process, or service by trade name, trademark, manufacturer, or otherwise does not necessarily constitute or imply its endorsement, recommendation, or favoring by the United States Government or any agency thereof, or Battelle Memorial Institute. The views and opinions of authors expressed herein do not necessarily state or reflect those of the United States Government or any agency thereof.

\author{
PACIFIC NORTHWEST NATIONAL LABORATORY \\ operated by \\ BATTELLE \\ for the \\ UNITED STATES DEPARTMENT OF ENERGY \\ under Contract DE-AC05-76RL01830
}

Printed in the United States of America

Available to DOE and DOE contractors from the

Office of Scientific and Technical Information,

P.O. Box 62, Oak Ridge, TN 37831-0062;

ph: (865) 576-8401, fax: (865) 576-5728

email: reports@adonis.osti.gov

Available to the public from the National Technical Information Service,

U.S. Department of Commerce, 5285 Port Royal Rd., Springfield, VA 22161

ph: (800) 553-6847, fax: (703) 605-6900

email: orders@ntis.fedworld.gov

online ordering: http://www.ntis.gov/ordering.htm

This document was printed on recycled paper.

$(8 / 00)$ 



\section{Wide Area Security Region Final Report}

$\begin{array}{ll}\text { YV Makarov } & \text { P Du } \\ \text { S Lu } & \text { TB Nguyen } \\ \text { X Guo } & \text { JW Burns }{ }^{(a)} \\ \text { JF Gronquist }^{(a)} & \end{array}$

March 2010

Prepared for the Bonneville Power Administration and the U.S. Department of Energy under Contract DE-AC05-76RL01830

Pacific Northwest National Laboratory Richland, Washington 99352

(a) Bonneville Power Administration 



\section{Abstract}

This report develops innovative and efficient methodologies and practical procedures to determine the wide-area security region of a power system, which take into consideration all types of system constraints including thermal, voltage, voltage stability, transient and potentially oscillatory stability limits in the system. The approach expands the idea of transmission system nomograms to a multidimensional case, involving multiple system limits and parameters such as transmission path constraints, zonal generation or load, etc., considered concurrently. The security region boundary is represented using its piecewise approximation with the help of linear inequalities (so called hyperplanes) in a multi-dimensional space, consisting of system parameters that are critical for security analyses. The goal of this approximation is to find a minimum set of hyperplanes that describe the boundary with a given accuracy. Methodologies are also developed to use the security hyperplanes, pre-calculated offline, to determine system security margins in real-time system operations, to identify weak elements in the system, and to calculate key contributing factors and sensitivities to determine the best system controls in real time and to assist in developing remedial actions and transmission system enhancements offline ${ }^{1}$. A prototype program that automates the simulation procedures used to build the set of security hyperplanes has also been developed. The program makes it convenient to update the set of security hyperplanes necessitated by changes in system configurations. A prototype operational tool that uses the security hyperplanes to assess security margins and to calculate optimal control directions in real time has been built to demonstrate the project success. Numerical simulations have been conducted using the full-size Western Electricity Coordinating Council (WECC) system model, and they clearly demonstrated the feasibility and the effectiveness of the developed technology. Recommendations for the future work have also been formulated.

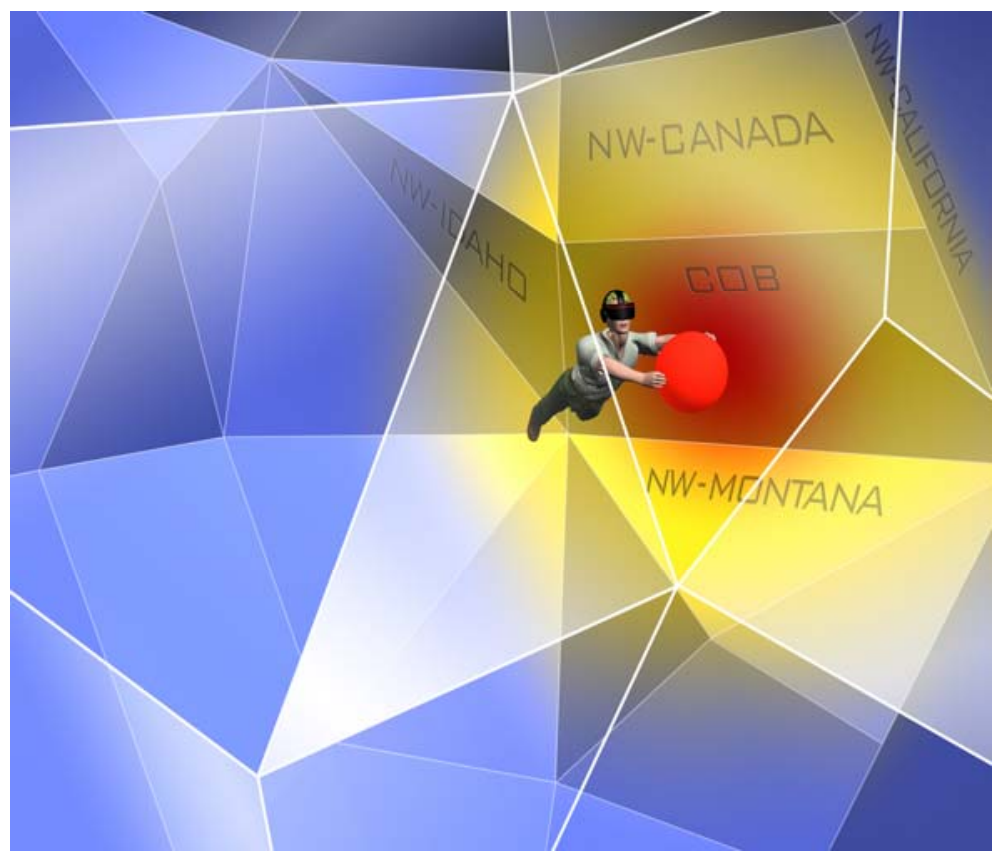

\footnotetext{
${ }^{1}$ The figure on this page shows a conceptual virtual reality representation of the power system dispatcher analyzing a current position of the operating points against the "walls" of a security region (by Michael C. Perkins, PNNL).
} 


\section{Executive Summary}

With the increasing penetration of intermittent renewable generation and the increasing variability of generation dispatches caused by variable resources and energy market forces, the power flow patterns become more and more diverse and deviate from the pre-designed conditions, imbedded into the existing transmission system structure and parameters. To mitigate the impacts of renewable resources on the system, new wide-area balancing authority (BA) coordination and cooperation schemes have been implemented or are currently under evaluation. Examples are the area control error diversity interchange program (ADI), the use of dynamics schedules for bringing more ancillary services into BAs' control areas, the actual consolidation of BAs, and others. These schemes could lead to wide-area redistributions of power flows and their growing additional variability. Additional considerations such as declining system inertia, compromised voltage stability characteristics, declining and non-uniform frequency response, and other new contributing factors influence the historically established transmission limits and force them to be calculated more frequently and adaptively. The rapidly developing phasor measurement system and relevant applications create new opportunities for quantifying power system secure operating conditions. For instance, the small signal stability margin becomes accessible in real time based on phasor measurements, and there are already substantial efforts in place to link it with the newly established transmission power flow limits. In this situation, it becomes evident that the transmission system constraints, which limit the variability of power flow patterns, should be revisited in terms of their definition, structure and numerical values. This necessity is addressed by this research work, co-funded by the Bonneville Power Administration (BPA) and the U.S. Department of Energy (DOE).

The work reported in this document develops a new methodology for calculating and representing transmission system constraints and the corresponding power system security region restricted by these constraints as a set of linear inequalities (so called hyperplanes) applied to the most important power system parameters, such as power flows on the critical paths, zonal generation and load, as well as nodal voltages and phase angles. These constraints can be summarized using sets of linear inequalities such as:

$$
\left\{\begin{array}{l}
\eta_{d, 1,1} d_{1}+\ldots+\eta_{d, 1, n} d_{n_{d}} \leq L_{d, 1} \\
\eta_{d, 2,1} d_{1}+\ldots+\eta_{d, 2, n} d_{n_{d}} \leq L_{d, 2} \\
\ldots \\
\eta_{d, m, 1} d_{1}+\ldots+\eta_{d, m, n} d_{n_{d}} \leq L_{d, m}
\end{array}\right.
$$

where $\eta_{d, i, j}$ are pre-calculated coefficients for a hyperplane $i ; L_{d, j}$ are the pre-calculated limits in each constraint, and $d_{j}$ are so called descriptor parameters, such as power flows in selected critical transmission paths.

The set (S-1) does not look very much different or more complicated mathematically comparing with the existing transmission system nomograms, where only 1,2 or 3 descriptor parameters are normally considered at the same time. Because the set (S-1) can incorporate $m$ parameters, this makes it a $m$ dimensional security nomogram. These constraints can be calculated and recalculated offline to reflect changing system configuration, the composition of committed power generation, and other significant changes. In particular, each of the system N-1 contingencies can produce its own set of inequalities, so that the inner intersection of all constraints will form a region of system secure operation. It is important 
that the framework developed in this report is flexible enough to incorporate all types of operating limits including thermal, voltage, voltage stability, small signal and transient stability limits.

As it was mentioned already, the existing two or three-dimensional nomograms and single transmission limits can be also interpreted as sets of linear inequalities of a limited dimension. When the number of dimensions is that limited, it becomes very difficult to reflect interactions between the multiple critical parameters actually influencing the system security margins. To eliminate the risk of violations, caused by representing the actual multidimensional problem with a limited number of descriptor parameters, the transmission planning and operations engineers have to build the nomograms under some rather conservative assumptions about the remaining parameters that are not included in a nomogram, or are not considered concurrently. This approach could lead to an underuse of the actual available transmission capacity and to an increase of the congestion management cost. The proposed methodology eliminates these problems.

The set of linear inequalities (S-1) could be represented as polyhedron in coordinates of $m$ descriptor parameters, as shown in Figure (S-1). An efficient offline procedure to build the approximated security region has been developed to produce a security region approximation in the $\mathrm{m}$-dimensional space. It is based on stressing the system in various directions and finding a first violation along each of these stress directions. The violations could be caused by thermal, voltage, voltage stability, transient stability, or small signal oscillatory stability constraints. Each stress direction additionally examined in the developed procedure produces $m$ new linear inequalities (this makes the procedure very efficient). The accuracy of approximation, achieved during the hyperplane building procedure, is controlled, so that the process stops when a given accuracy has been achieved.

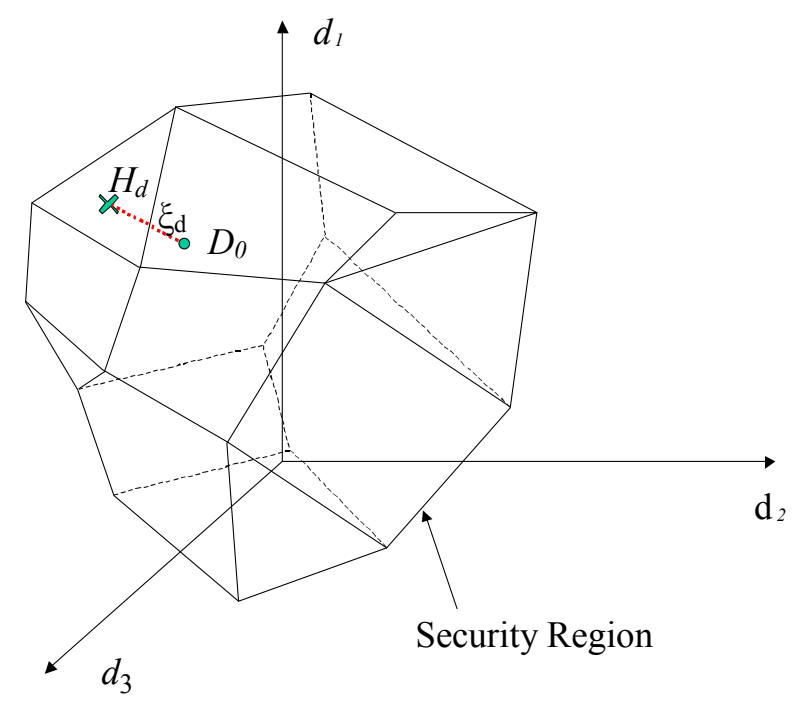

Figure S- 1. Conceptual View of the Security Region Approximated by Linear Inequalities

In real time, the set of linear inequalities (S-1) can be efficiently used for the following purposes:

- Fast and convenient assessment of the system security conditions. Having constraints (S-1) precalculated offline for each analyzed contingency, it becomes very easy to determine the following important characteristics in real time: 
- Whether the operating point $D_{0}$ is inside or outside the security region (This can be done by making sure that all approximating inequalities are satisfied),

- Which constraints are violated (This is done by identifying violated inequalities), and

- What the most limiting constraints are (This is done by calculating the distance from the current operating point $D_{0}$ to the approximating planes $H_{d}$ ).

- Easy-to-calculate security margin. The distance $\xi_{d, i}$ from the current operating point $D_{0}$ to the nearest constraint hyperplane $H_{d, i}$ determines the security margin. The resulting stability margin corresponding to the minimum distance from the stability boundary, i.e., the distance to the closest constraint facet, must be greater than certain minimum security margin $\mu_{d}$ :

$$
\min _{(i)} \xi_{d, i} \geq \mu_{d}
$$

- Selecting better controls and remedial actions. If the security margin is not sufficient, it should be increased by applying controls helping to move the operating point away from the nearest facet $H_{d, i}$ along the vector $\xi_{d}$.

- Identification of weak elements influencing the security margin. The closer the direction of $\xi_{d}$ is to some coordinates of the nomogram, the more influential these parameters are. This idea helps to identify weak parameters in the system that are responsible for the insufficient security margin.

- Refining operating transfer capability limits. The linear constraint set (S-1) establishes limits on simultaneous power transfers in multiple paths in the system. It helps to find a more flexible, more adaptive, and hopefully less conservative description of the system security limits.

Numerical simulations were carried out on the full-size WECC system model, which consisted of 15,334 buses and 3,034 generators. The WECC 2007 heavy summer case, provided by BPA, was used as the base case for this study. Based on the engineering experience of BPA's engineers, a subset of the WECC system parameters has been identified to be used as descriptor and control parameters helping to approximate the security boundary of a subsystem of WECC and to develop multidimensional nomograms for this region. They are as follows:

Descriptor Parameters (power flows):

1. Flow $1 d_{1}$

2. Flow $2 d_{2}$

3. Flow $3 d_{3}$

4. Flow4 $d_{4}$

5. Flow5 $d_{5}$

6. Flow6 $d_{6}$

7. Flow $7 d_{7}$ 


\section{Flow8 $d_{8}$}

Control Parameters (area load or generation):

1. Areal generation $c_{1}$

2. Area2 generation $c_{2}$

3. Area3 generation $c_{3}$

4. Area4 generation $c_{4}$

5. Area5 generation $c_{5}$

6. Area6 load $c_{6}$

7. Area7 load $c_{7}$

8. Area8 load $c_{8}$

9. Area9 generation $c_{9}$

A prototype automatic procedure to build the approximated security region has been developed. It has three components: (1) a procedure to find the boundary points, (2) a procedure to build the hyperplanes, and (3) procedure to refine the hyperplanes. Ninety two hyperplanes have been found for the model. The selected tangent hyperplanes were validated in two ways

a. By measuring the distance from the hyperplanes to the actual security boundary.

b. By comparing the hyperplanes with the existing nomograms currently used by BPA.

Extensive numerical experiments demonstrated the validity of the proposed methodology and its capability of quantifying power transfer limits and security margins. The comparison between the hyperplanes built in this project and the existing nomograms showed that the proposed approach, which considered more system variables at the same time, was less conservative and more accurately represented the system security boundary.

The future work being planned includes:

1. Combine offline computer simulations runs for the system model with processing of real-time power system measurements, including Supervisory Control and Data Acquisition (SCADA) and state estimation data, as well as subsecond synchrophasor data, for a quick evaluation of the real-time system security margin.

2. Evaluate different options (such as generator drop, load shedding, capacitor switching, etc.) to increase the security margin. These controls could be incorporated into the proposed framework as automatic remedial actions or serve as advisory tools to operators.

3. Refine the procedures, developed to build security hyperplanes, to cover the entire control and description parameter space, and validate the resulting hyperplanes built for the BPA power system. Initially, the resulting hyperplane sets could be used by BPA in parallel with the existing nomograms. With the increasing confidence in the newly proposed methodology, it can be later applied to refine the existing nomograms and transmission limits. 
4. Further develop the automated simulation program for offline building the security hyperplane sets to increase the degree of automation in the entire process, and to include oscillatory and transient stability limits.

5. Develop functionalities of the operational tool that apply security constraints obtained by offline computer simulations to real-time SCADA and subsecond phasor measurement unit data, for online security assessment. For example, when calculating optimal control directions, consider all types of control capabilities in the system instead of only area load and generation, feasibility of the control direction under the particular operating condition, and operator preferences.

6. Develop a specification for building an industrial-grade tool out of the methodologies and procedures developed in the project.

7. Use the methodology to benchmark system behavior based on phasor measurements (which is the highest priority for North American SynchroPhasor Initiative (NASPI)).

8. Provide technology transfer to BPA and wider dissemination of the project results. 


\section{Acknowledgments}

The work has been sponsored by Technologies Innovation Department at the Bonneville Power Administration (BPA). BPA's Project Manager is James W. Burns. The project was co-sponsored by the Office of Power Technologies at the U.S. Department of Energy through the Consortium of Electric Reliability Technology Solutions (CERTS). DOE's Project Manager was Phillip N. Overholt. CERTS Project Manager was Dr. Joseph H. Eto.

The project team is deeply grateful to the following researchers and managers who contributed significantly to the success of this project.

Bonneville Power Administration:

William A. Mittelstadt

Department of Energy:

Philip N. Overholt, Office of Power Technologies

University of Wisconsin-Madison:

Prof. Ian Dobson

Pacific Northwest National Laboratory:

Evan O. Jones - Product Line Manager

Carl H. Imhoff - Manager, Electric Infrastructure Sector

Jeffery E. Dagle - Chief Engineer

Mark P. Morgan - Technical Group Manager, Energy Technology Development

Ron Melton, Technical Team Lead, Electrical Power Systems Integration

Ross Guttromson, Technical Team Lead, Electrical Power Systems Engineering

Susan J. Arey - Project Specialist

Sheena L. Kanyid - Contracting Officer

Technical University of Denmark

Hjortur Johannsson, visiting student 



\title{
Notations
}

\author{
$B_{c}(D)$ \\ A function expressing the security boundary (multidimensional \\ nomogram) in coordinates of controlled parameters. \\ $B_{d}(D)$ \\ A function expressing the security boundary (multidimensional \\ nomogram) in coordinates of descriptor parameters. \\ $B_{p}(P)$ \\ $c$ \\ c \\ $C=\left[c_{1}, c_{2}, \ldots, c_{n_{c}}\right]^{t}$ \\ $\bar{C}=\left[\bar{c}_{1}, \bar{c}_{2}, \ldots, \bar{c}_{n_{c}}\right]^{t}$ \\ $C_{0}=\left[c_{0,1}, c_{0,2}, \ldots, c_{0, n_{c}}\right]^{t}$ \\ $C_{d}$ \\ $C_{p}$ \\ $d$ \\ $\bar{d}$ \\ $D=\left[d_{1}, d_{2}, \ldots, d_{n_{d}}\right]^{t}$ \\ $\bar{D}=\left[\bar{d}_{1}, \bar{d}_{2}, \ldots, \bar{d}_{n_{d}}\right]^{t}$ \\ $D_{0}=\left[d_{0,1}, d_{0,2}, \ldots, d_{0, n_{d}}\right]^{t}$ \\ A function expressing the security boundary (multidimensional \\ nomogram) in coordinates of independent system parameters. \\ Controlled parameter. Controlled parameters are system parameters that \\ can be varied by the system operator. \\ Controlled parameter at the security boundary point. \\ Vector of controlled parameters. \\ Vector of controlled parameters at the security boundary point. \\ Vector of controlled parameters at the base power flow point. \\ Control vector in the descriptor parameter space. \\ Control vector in the independent parameter space. \\ Descriptor parameter. Descriptor variables reflect the most influential or \\ understandable combinations of parameters that influence the system \\ security margin. \\ Descriptor parameter at the security boundary point. \\ Vector of descriptor variables reflecting the most influential or \\ understandable combinations of parameters that influence the system \\ security margin. \\ Vector of descriptor variables at the security boundary point. \\ Vector of descriptor variables at the base power flow point.
}


Vector or a $n_{p} \times n_{d}$ matrix of coefficients describing a linear combination of independent system parameters forming a descriptor parameter, $D=D P^{t} \cdot P=S P^{t} \cdot\left(P_{0}+\alpha \cdot \Delta P\right)$.

$H_{c}$

$H_{d}$

$H_{p}$

$L_{c}$

$L_{d}$

$L_{p}$

$n_{c}$

$N_{c}$

$n_{d}$

$N_{d}$

$n_{p}$

$N_{p}$

$p$

$P=\left[p_{1}, p_{2}, \ldots, p_{n_{p}}\right]^{t}$
Approximating hyperplane in the controlled parameters space.

Approximating hyperplane in the descriptor parameters space.

Approximating hyperplane in the independent system parameters space.

A constant used in approximating hyperplane in the controlled parameters space.

A constant used in approximating hyperplane in the descriptor parameters space.

A constant used in approximating hyperplane in the independent system parameters space.

Number of controlled parameters.

Normal vector to a hyperplane, approximating security region boundary in coordinates of controlled parameters.

Number of descriptor parameters.

Normal vector to a hyperplane, approximating security region boundary in coordinates of descriptor parameters.

Number of independent system parameters.

Normal vector to a hyperplane, approximating security region boundary in coordinates of independent system parameters.

Independent system parameter, such as nodal injections of active or reactive power.

Independent system parameters vector, such as nodal injections of active and reactive power. 


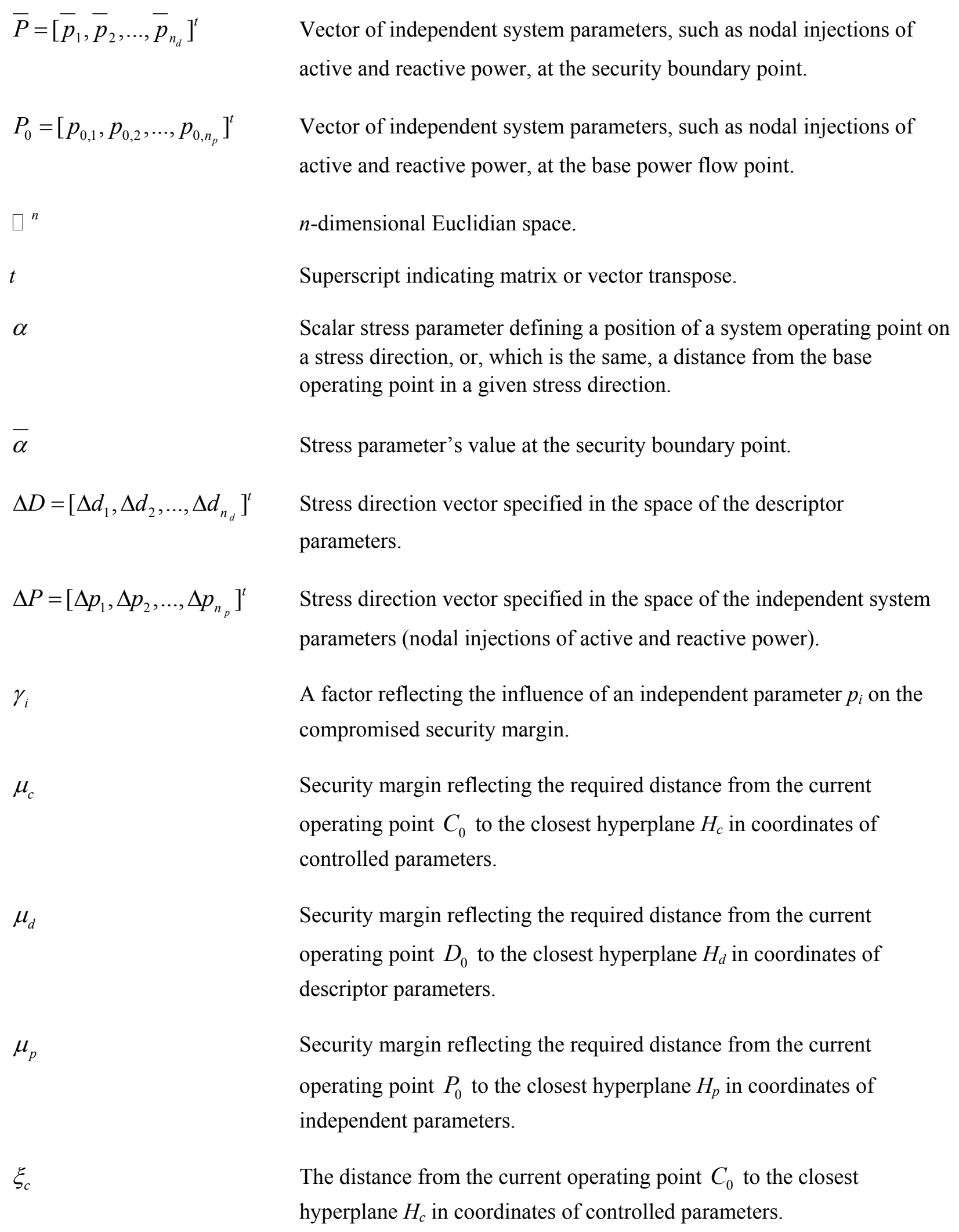

The distance from the current operating point $C_{0}$ to the closest hyperplane $H_{c}$ in coordinates of controlled parameters. 
The distance from the current operating point $D_{0}$ to the closest hyperplane $H_{d}$ in coordinates of descriptor parameters. The distance from the current operating point $P_{0}$ to the closest hyperplane $H_{p}$ in coordinates of independent system parameters. 


\section{Contents}

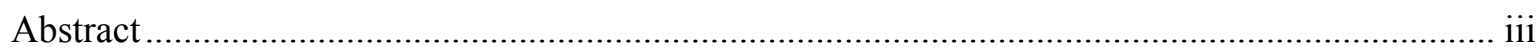

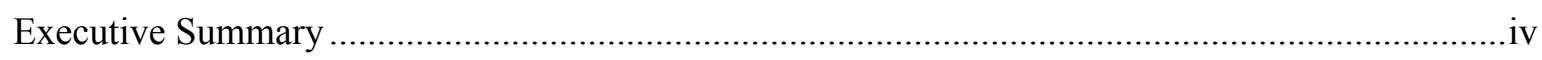

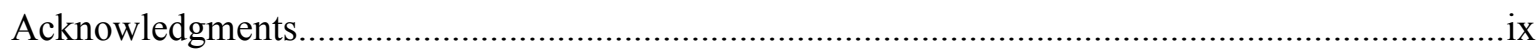

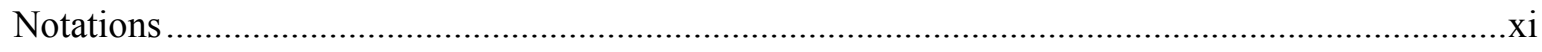

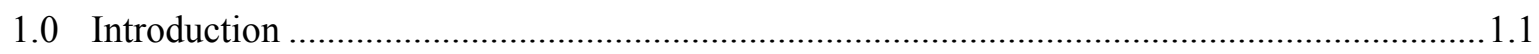

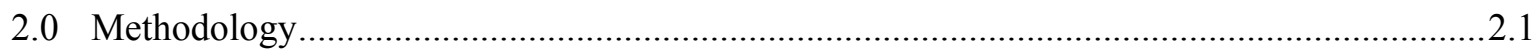

2.1 Concepts of Stress Direction, Descriptor Variables, State and Parameter Spaces ...........2.1

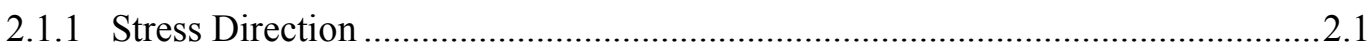

2.1.2 Descriptor, State, and Independent System Parameter Spaces............................2.1

2.1.3 One-dimensional System Security Margin [12] ..............................................2.2

2.1.4 Two-dimensional System Security Nomogram $[12]^{2}$.....................................2.3

2.1.5 Relation Between Parameter Space and Nomogram Normal Vectors $[12]^{2}$........2.4

2.2 Finding Hyperplanes, Their Normal Vectors, and Sensitivities...................................2.5

2.2.1 Tangent Hyperplane Definition Using Normal Vector ........................................2.5

2.2.2 Secant Hyperplane Definition Using $n$ Point Approach......................................2.6

2.2.3 Finding Secant Hyperplanes with Specified Security Margin [12] ......................2.7

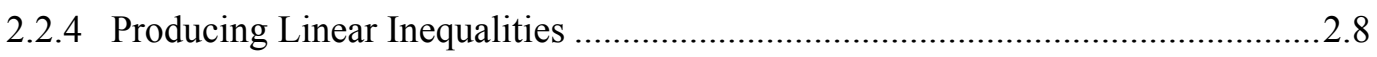

2.2.5 Procedure to Calculate Sensitivities of the Security Boundary Points .................2.8

2.3 Building the Security Region in a Multi-dimension Parameter Space ...........................2.9

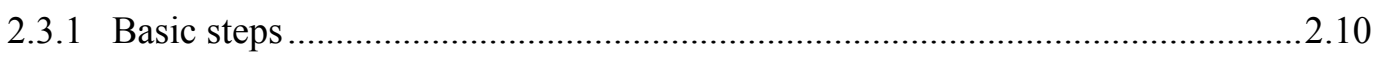

2.3.2 Determine the Region of Interest in the Description, Control, and Independent System Parameter Spaces ..........................................................................2.11

2.3.3 Starting Point and Stress Directions to Find Initial Hyperplanes.......................2.13

2.3.4 Developing the Set of Hyperplanes Based on a Given Accuracy of

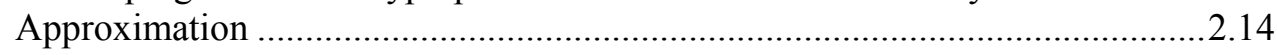

2.3.5 Stressing the System to Find the Security Boundary Points ..............................2.15

2.4 Security Region Description and Security Margin.................................................... 2.16

2.4.1 Security Region Description Using a Set of Linear Inequalities .......................2.16

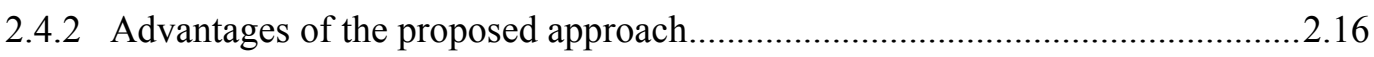

2.5 Most Effective Controls, Weak Elements, and Remedial Actions.................................2.17

2.5.1 Critical and Subcritical Distances and Compromise Control............................2.18

2.5.2 An Illustration of the Critical and Subcritical Distances and Compromise

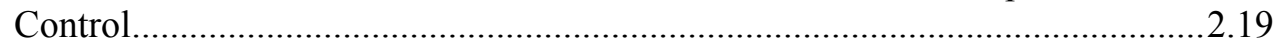

2.5.3 Selecting Controls and Remedial Actions in the Controlled or Independent

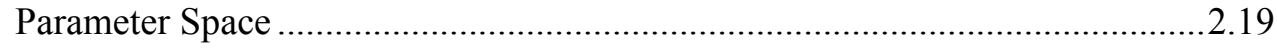

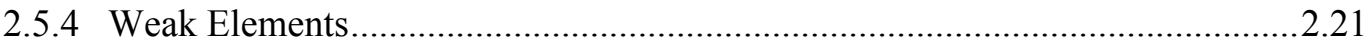

2.6 Refining Operating Transfer Capability (OTC) Limits Using Multidimensional Nomograms .... 


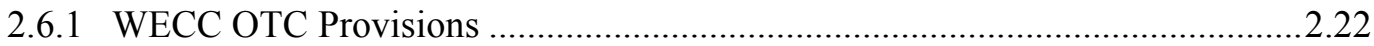

2.6.2 Drawbacks of the Existing Approach to Determine the OTC ...........................2.2.23

2.6.3 New Approach to Determine OTC Based on $n$-dimensional Nomograms ........2.24

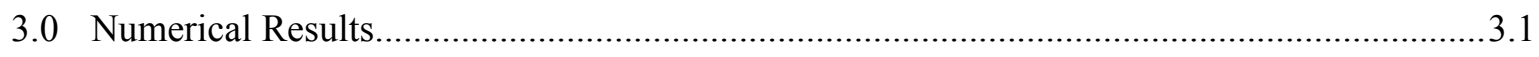

3.1 Descriptor and Control Parameters and Stress Patterns ............................................... 3.1

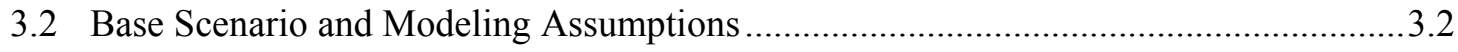

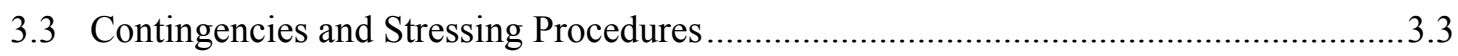

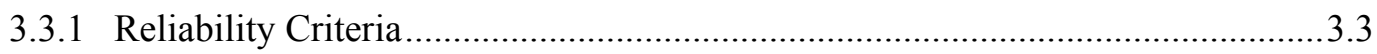

3.3.2 Simulation Procedures to Find Security Boundary Points ....................................3.5

3.3.3 Simulation Procedures to Build Security Region in a Multi-dimension Parameter Space .................................................................................................. 3.10

3.3.4 Automatic Simulation Procedures in PowerWorld SimAuto ...............................3.10

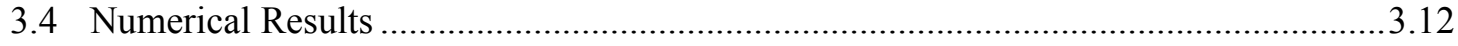

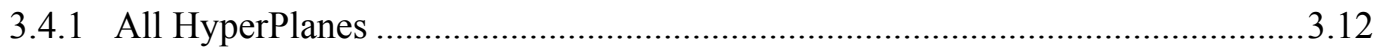

3.4.2 Validation of Tangent Hyperplanes ..................................................................12

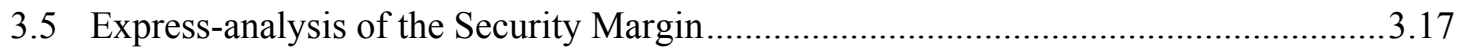

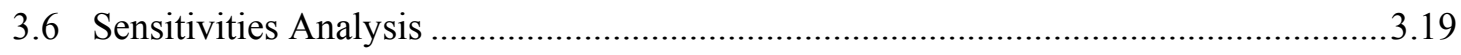

3.7 Real-time Security Assessment Application ............................................................. 3.21

3.8 Evaluate Transformation of Hyperplanes from Power Flow Space to Angle Space......3.22

4.0 Conclusions and Future Work ........................................................................................ 4.1

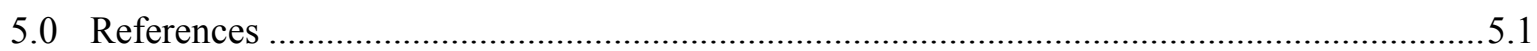

Appendix A Literature Review on the Security Region Concept for Power Systems .................... A.1

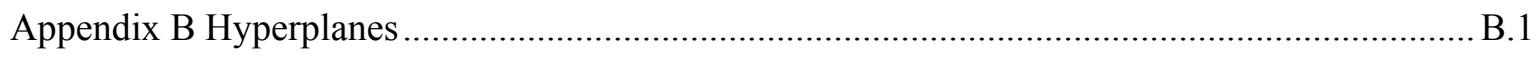

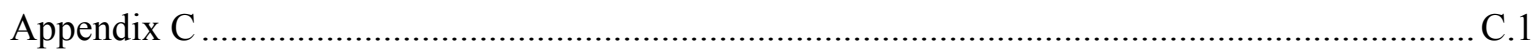

Evaluate Different Transformation of Hyperplanes in Angle Space ......................................... C.1 


\section{Figures}

Figure S- 1. Conceptual View of the Security Region Approximated by Linear Inequalities...........v

Figure 2.1. Illustration of an Approximating Hyperplane in the Descriptor Parameter Space .......2.5

Figure 2.2. Internal Approximation of the Security Region in the Space of Descriptor Parameters 2.7

Figure 2.3. Illustration of Security Boundary in the Descriptor Space ......................................2.8

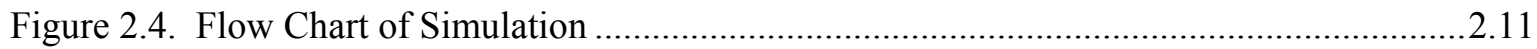

Figure 2.5. Security Boundary in the Area of Interest in a Three-dimensional Descriptor Space 2.12

Figure 2.6. Stressing the System to Find Initial Hyperplanes ....................................................2.14

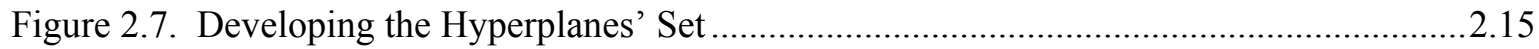

Figure 2.8. Conceptual View of the Approximated Security Region ........................................2.17

Figure 2.9. An Illustration of the Critical and Subcritical Distances and Compromise Control...2.19

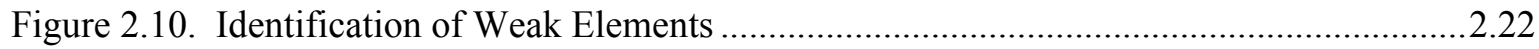

Figure 2.11. Example of three-dimensional Nomogram.......................................................2.24

Figure 3.1. Non-uniqueness of the Normal Vector for Interdependent Controlled Parameters......3.2

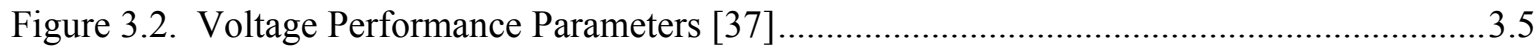

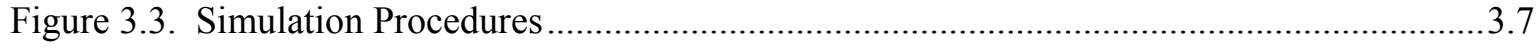

Figure 3.4. Flowchart of Refined Simulation Steps to Find a Boundary Point............................... 3.9

Figure 3.5. Automatic Simulation Procedures in PowerWorld SimAuto ....................................3.11

Figure 3.6. 2007 Heavy Summer Nomogram Predicted from Hyperplane ...................................3.14

Figure 3.7. Three-dimensional View of 2007 Summer Nomogram Projected from Hyperplane 3.15

Figure 3.8. 2008 Summer Nomogram Provided by BPA .....................................................16

Figure 3.9. Three-dimensional View of 2008 Summer Nomogram Provided by BPA ................3.17

Figure 3.10. Calculation of Security Margin ...................................................................................18

Figure 3.11. Sensitivities of Change of Generation/Load with Respect to Security Margin ........3.19

Figure 3.12. Sensitivities of Hyperplane in Descriptor Parameter Space ....................................3.20

Figure 3.13. Comparison between the Hyperplanes with Different Power Flow in the Interface of

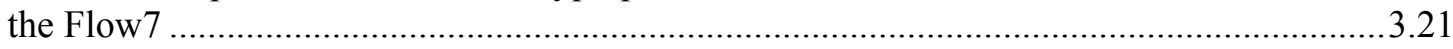

Figure 3.14. Convention Online Security Assessment................................................................22

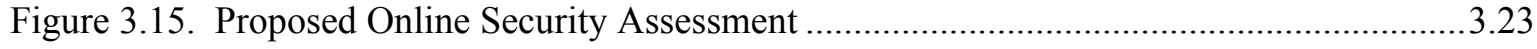

Figure 3.16. Real-time Application of Hyperplanes to Monitor Stability Margin........................3.24

Figure A. 1. Probabilistic Community Activity Room ............................................................

Figure A. 2. SDSR's Boundary Versus Percentage of Induction Motor...................................... A.3

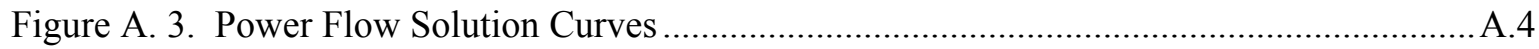

Figure A. 4. Predictor-corrector Process................................................................................... A

Figure A. 5. Critical Cut-set Identification (the weak nodes are mostly located on the terminal of long transmission lines without reactive power support).................................................... 
Figure A. 6. Voltage Stability Boundary (hyperplane) (a: stability boundary in critical cut-set space; b: 2-dimension feasible region with thermal and voltage stability limits)

Figure A. 7. Boundary of Secure Operating Region for Two Simultaneous Transfers ................. A.8

Figure A. 8. RTVSA Algorithms Flowchart .......................................................................

Figure A. 9. The Boundary of Stability Region ................................................................... A.11

Figure A. 10. Equipotential Energy Surfaces for Three-Machine System................................ A.13

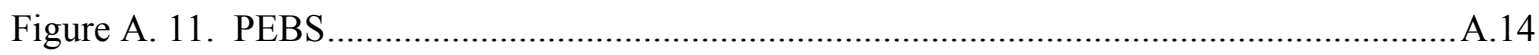

Figure A. 12. Three Main Computational Tasks in the BCU Method .......................................14

Figure A. 13. Three-dimensional Illustration of Security Region for Equivalent System [10] ...A.16

Figure A. 14. Singular Points and Trajectories of Two Machines ................................................16

Figure A. 15. Singular Points, Separatrix, and Tangent Hyperplanes ....................................... A.17

Figure C. 1. Power Flow on the Interface between Neighboring areas I and J........................... C.1

Figure C. 2. Distribution of Relative Errors Caused by Approximation in (C.5) ......................... C.4

Figure C. 3. Distribution of Relative Errors Caused by Approximation in (C.6) ........................ C.4 


\section{Tables}

Table 2-1. Region of Interest'

.2 .13

Table 3-1. WECC Disturbance-Performance Table of Allowable Effects on Other Systems [39] .3.4

Table 3-2. Normal Vectors of Hyperplanes in the Control Parameter Space

..3.12

Table 3-3. Normal Vectors of Hyperplanes in the Descriptor Parameter Space.

Table 3-4. Calculation of Security Margin for Operation Point $D_{0}$ 3.18

Table B- 1. Coefficients of hyper-planes B. 1

Table C- 1. Coefficients of A Hyperplane in the Tie-line Power Flow Space. C. 3 


\subsection{Introduction}

Security region is defined as a set of load demands and power generations (in the controlled parameter space) or voltages and their phase angles (in the state variables space) for which the power flow equations and the security constraints are satisfied [1][2]. The security region and associated security margin concepts are summarizing challenges that power system planners and operators face to ensure reliable power operations [3]. Generally, the security region boundary is a nonlinear function of system parameters, security constraints imposed, system configuration, and contingencies involved in the analysis. The security region can represent thermal, voltage, voltage stability [4][5][6], small signal [7], or transient stability constraints [8][9][10][11].

Considerable work has been devoted to various aspects of security and transfer limits calculation in electric power systems. There is a progression from one-directional methods estimating the voltage stability margin in a specified direction to multidirectional methods evaluating the distances to instability, and further from the multidirectional methods to the methods exploring the entire voltage security region in the parameter space [12]. Appendix A provides an extended review of existing approaches developed for these tasks. The main approaches can be listed as follows.

Professor F. Wu and Kumagai were among the first researchers who suggested a region-wise (settheoretic) approach to power system security analysis [1]. This approach characterizes the set of all steady-state secure operating points by using various operating and security constraints applied to the operating vectors.

The computation of voltage stability security limits in the multi-coordinate parameter space has been also discussed in several other works. Multiple voltage stability indices and methods for finding and quantifying the voltage stability margin have been suggested. The most common methods include the minimum singular value approach, point of collapse method, optimization methods, and continuation power flow, and repeated power flow [12][13][14][15][16][17]. For example, Professor Y. Yu proposed representation of security region in the critical cut-set space [4]. In this approach, after the weak nodes are identified, the continuation power flow $(\mathrm{CPF})$ is used to build the approximating hyperplanes. The physical and operational margins (POM) approach was used to identify the boundary of the secure operating region for East Kentucky Power Cooperative [18] and for some other systems. The boundary of the operating region (BOR) was shown as a projection onto different planes, such as power injections, load and generation, interface and/or tie-line flows, as well as a three-dimensional representation. The real-time voltage security assessment (RTVSA) approach used a variant of the CPF method [12][19]. At the point of collapse (PoC), the left eigenvector of the power flow Jacobian matrix was calculated to estimate the tangent hyperplane with respect to the load flow feasibility boundary. Procedures have been developed to "orbit" the boundary and select the set of cut-set planes needed to approximate it. If the feasibility boundary is convex, the hyperplanes could represent the secure operating region with manageable accuracy. 
One of the important problems for the concept of the power system security region is the adequate description of its boundary. There is a need to develop an analytical description and/or approximation of the boundary. The analytical description usually means the use of linear or nonlinear inequalities applied to a certain number of critical parameters such as power flows, load levels, voltage magnitudes, etc. If all inequalities are satisfied, the analyzed operating point is considered to be inside the security region. If any of the inequalities is violated, the operating point is considered to be outside of the security region. The approximation means a sort of interpolation between the boundary points obtained. It can be used as a part of the analytical boundary description (for the automatic security margin assessment systems), or separately for the purposes of visualization. The simplest approximation uses linear inequalities. The first known use of the approximation was apparently related to the operating nomograms - see [20] for more details. The operating nomograms are usually represented as piecewise linear contours on a plane of two critical parameters. If three critical parameters are involved, the nomogram is represented by a family of contour lines; each of the contours corresponds to a certain value of the third parameter. It becomes difficult to represent a nomogram for four or more critical parameters. The natural extension of the linearized stability nomograms for three or more critical parameters is based on the use of hyperplanes - the planes that are defined in the multidimensional parameter space as approximations of the stability boundary. These efforts are described in [12][19] (voltage stability boundary approximation), [8][11][17] (transient/dynamic stability boundary approximation), and other works. A nonlinear approximation was also successfully used to provide an analytical description of the stability boundary [21][22]. These approaches employ quadratic inequalities. The inequalities are applied to the nodal power injections, cutset power flows, and other parameters.

The artificial neural network (ANN) model de facto has been used to provide an approximation of the security boundary [23]-[36]. The idea behind the techniques based on ANN is to select a set of critical parameters such as power flows, loads, and generator limits, and then train an ANN on a set of simulation data to estimate the security margin. The advantages of the ANN models include their ability to accommodate nonlinearities and their very fast performance in real time. At the same time, there are difficulties associated with building the training datasets and ANN training.

Despite innovative and fruitful research and development efforts in the security assessment area, power system operators and planners still have needs in more adequate, accurate and useable methodologies. The utilization of the approximated security region approach provides significant advantages for developing on-line applications, but still the security limit conditions tend to be very conservative because of the usually limited number of critical parameters employed to describe these security nomograms. The multidimensional representation of system security conditions reduces that conservatism, increases the accuracy and robustness of the approximation, expands the range of admissible operating conditions and makes the security limits more adaptive to changing system conditions.

The wide-area phasor measurement networks combined with the SCADA measurements now open up unprecedented possibilities for providing system operators with better tools for monitoring system vulnerability and finding remedial actions in real time. The proposed approach intends to provide widearea real-time operational awareness, security monitoring, and control advice that will enable BPA operators and WECC security coordinators to quickly determine whether the WECC system is currently in, or close to being in trouble, and what corrective actions should be taken. 
This report is organized as follows. Section 2 discusses the basic concept and procedures to determine the security region hyperplanes and the normal vectors. The numerical simulation results are presented in Section 3. Section 4 summarizes the conclusions and future work. 



\subsection{Methodology}

\subsection{Concepts of Stress Direction, Descriptor Variables, State and Parameter Spaces}

This section explains the basic concepts used in the methodology.

\subsubsection{Stress Direction}

Stress direction (procedure) specifies how the system parameters should be changed from their base values as a function of a scalar stress parameter. For example, the amount of total generation and load change can give a scalar amount of stress, while the relative change of individual generation and load (participation factors) can help to define a stress direction. Each specific direction and value of the stress parameter must uniquely define the system state. This implies certain fixed patterns for varying the system generation and loads (for example, load participation factors, sequence of generator dispatch, distributed slack bus model for unbalanced stresses, and others). Stress directions can include some local system stresses addressing a particular problem area, and global stresses such as the wide-area generation re-dispatch in the system.

\subsubsection{Descriptor, State, and Independent System Parameter Spaces}

Descriptor variables reflect the most influential or understandable combinations of parameters (or derivative parameters) that influence the system security margin. Examples are the total area load, power flows in certain system paths, total generation, and others (the system operating nomograms' coordinates are good examples of descriptor parameters). In the simplest case, descriptor parameters can include some primary system parameters such as nodal voltages and nodal power injections. Descriptor variables help to adequately address global and local system security margins without involving thousands of primary parameters. Certain subsets of descriptor variables can correspond to some local system security areas.

The state space includes all system nodal voltage magnitudes and voltage phase angles.

The independent system parameter space includes all nodal power injections (for P-Q buses) and real power injections and voltage magnitudes (for P-V buses).

The system security boundary can be comprehensively (and uniquely) described in the independent system parameter space (and the state space), but in this case, the description would involve thousands of variables. Descriptor parameters help to reduce the dimensionality of the problem by considering the most influential combinations of parameters (or derivative parameters).

The descriptor parameter space includes all descriptor parameters. Because the points in the descriptor parameter space can be mapped into the points of the independent system parameter and state parameter spaces in many different ways (because of the limited number of descriptor parameters space 
dimensions), certain fixed system stress procedures should be introduced to make this mapping adequate and unique [12][19].

A critical descriptor parameter set must satisfy the following criteria [20]:

1. Sufficiency: The parameters must contain sufficient information to allow prediction of the performance measure within a desired accuracy for all operating conditions within the study scope.

2. Cardinality: The critical parameter set should be chosen as the set of minimum size that satisfies the set sufficiency criterion.

3. Controllability: At least one critical parameter within the set must be controllable by the operator so that the operating point can be adjusted with respect to the boundary using preventive actions.

\subsubsection{One-dimensional System Security Margin [12]}

We specify the pattern, or participation of all injections in a column vector $\Delta P$. Then the changes in injections are $\alpha \cdot \Delta P$, where $\alpha$ is a scalar parameterization of the system stress. If the base case parameters are $P_{0}$, the stressed system parameters parameterized by $\alpha$ is the column vector

$$
P=P_{0}+\alpha \cdot \Delta P
$$

It can be useful to normalize $\Delta P$ so that $\alpha$ is expressed in some convenient way and in convenient units. For example, if the parts of $\Delta P$ corresponding to generator injections are normalized to have $L 1$ norm, $\alpha$ measures the generation margin in $L 1$; that is, the sum of the generation increases.

A bulk change descriptor variable $d$ is a quantity such as an area load increase or an import across a cut set. Variable $d$ is (for a given network structure) an affine function of the independent system parameters $P$ so that ${ }^{3}$

$$
d=D P^{t} \cdot P=D P^{t} \cdot\left(P_{0}+\alpha \cdot \Delta P\right)
$$

where $D P$ is a vector of coefficients describing a linear combination of system parameters forming a descriptor parameter $d,{ }^{4}$ and superscript $t$ indicates matrix transpose.

In Equation (2.2), $D P$ is a fixed vector that can be computed from the network equations. For example, $D P$ for a cut-set flow $d$ is computed by summing line flows for lines in the cut set as a function of injections. At the security limit, we get:

$$
\bar{d}=D P^{t} \cdot\left(P_{0}+\bar{\alpha} \cdot \Delta P\right)
$$

If we assume a fixed stress direction or injection participation $\Delta P$, then $\alpha$ is also a function of $d$ :

\footnotetext{
${ }^{2}$ Used with permission from Prof. Ian Dobson, University of Wisconsin-Madison.

${ }^{3}$ Note that the matrix multiplication $D P^{t} \cdot\left(P_{0}+\alpha \cdot \Delta P\right)$ is the same as the dot product between $D P$ and $\left(P_{0}+\alpha \cdot \Delta P\right)$. (This formula assumes that the base case $d_{0}=D P^{t} \cdot P_{0}$, but this can be easily generalized as needed by adding a suitable constant).

${ }^{4}$ Matrix $D P$ is chosen so that $D$ is expressed in convenient units such as MW of import through the cut set or MW of load.
} 


$$
\alpha=\frac{d-D P^{t} \cdot P_{0}}{D P^{t} \cdot \Delta P}
$$

Specifying $d$ and $\Delta P$ now defines the system stress $\alpha$ and $\Delta P$. That is, the system stress can be specified by the amount of a bulk change of injections $d$ and also by assuming the pattern, or participation of all injections in the column vector $\Delta P$.

In summary, we can formulate the one-dimensional margin to voltage collapse as follows:

- We make the assumption of the participation factors $\Delta P$.

- Then we can specify an amount of stress by descriptor $d$, and

- The margin to the security boundary can be specified by the descriptor margin $d^{*}=D P^{t} \cdot\left(P_{0}+\alpha^{*}\right.$. $\Delta P)$.

\subsubsection{Two-dimensional System Security Nomogram $[12]^{2}$}

Here we define two bulk change descriptor variables $d_{1}$ and $d_{2}$ so the descriptor variables are the column vector $D=\left(d_{1}, d_{2}\right)^{t}$. Now $D P$ is a function given by the matrix $D P=\left[D P_{1}, D P_{2}\right]$, which has a dimension of (number of parameters) $\times 2$, so that

$$
D=\left(d_{1}, d_{2}\right)^{t}=D P^{t} \cdot P=D P^{t} \cdot\left(P_{0}+\alpha \cdot \Delta P\right)
$$

The nomogram curve $B_{d}(D)$ is given by $\left(\bar{d}_{1}, \bar{d}_{2}\right)^{t}=D P^{t} \cdot\left(P_{0}+\bar{\alpha} \cdot \Delta P\right)$ as $\Delta P$ varies. $^{5} \mathrm{We}$ assume that the nomogram curve is given (locally) by:

$$
B_{d}(D)=B_{d}\left[\left(d_{1}, d_{2}\right)\right]=0
$$

If we now assume two fixed stress directions or injection participations $\Delta P_{1}$ and $\Delta P_{2}$, so that

$$
P=P_{0}+\alpha_{1} \cdot \Delta P_{1}+\alpha_{2} \cdot \Delta P_{2}
$$

then

$$
D=\left(d_{1}, d_{2}\right)^{t}=D P^{t} \cdot\left(P_{0}+\alpha_{1} \cdot \Delta P_{1}+\alpha_{2} \cdot \Delta P_{2}\right)=D_{0}+M \cdot\left(\alpha_{1}, \alpha_{2}\right)^{t}
$$

where $M$ is the $2 \times 2$ matrix, $M=\left[D P^{t} \cdot \Delta P_{1}, D P^{t} \cdot \Delta P_{2}\right]$.

Then $\left(\alpha_{1}, \alpha_{2}\right)^{t}$ and $P$ are also affine functions of $\left(d_{1}, d_{2}\right)^{t}$ :

$$
\begin{gathered}
\left(\alpha_{1}, \alpha_{2}\right)^{t}=M^{-1} \cdot\left[\left(d_{1}, d_{2}\right)^{t}-D P^{t} \cdot P_{0}\right] \\
P=P_{0}+\left(\Delta P_{1}, \Delta P_{2}\right) \cdot\left(\alpha_{1}, \alpha_{2}\right)^{t}
\end{gathered}
$$

\footnotetext{
${ }^{5}$ Note that $\bar{\alpha}$ is a function of $\Delta P$.
} 


$$
P=P_{0}+\left(\Delta P_{1}, \Delta P_{2}\right) \cdot M^{-1} \cdot\left(D-D_{0}\right)
$$

Specifying $\left(d_{1}, d_{2}\right)^{t}$ and $\Delta P_{1}$ and $\Delta P_{2}$ now defines the system stress in terms of injections $P$. That is, the system stress can be specified by the amount of a bulk change of injections $\left(d_{1}, d_{2}\right)^{t}$, and also assuming the patterns, or participations of all injections in the column vectors $\Delta P_{1}$ and $\Delta P_{2}$.

In summary, we can formulate a two-dimensional margin to the security boundary, which is the same as nomogram $B_{d}(D)=B_{d}\left[\left(d_{1}, d_{2}\right)\right]=0$, as follows:

We make the assumption of the participation factor vectors $\Delta P_{1}$ and $\Delta P_{2}$. Then we can specify an amount of stress by descriptor vector $D=\left(d_{1}, d_{2}\right)^{t}$. The security boundary can be specified by the curve $B_{d}(D)=0$ in the nomogram. It is important to note the dependence of the nomogram curve on the choice of $\Delta P_{1}$ and $\Delta P_{2}$.

\subsubsection{Relation Between Parameter Space and Nomogram Normal Vectors [12] ${ }^{2}$}

The descriptor parameters $D$ considered here are affine functions of the parameter space parameters $P$ given by

$$
D=D_{0}+D P^{t} \cdot P
$$

where $D P^{t}$ is a matrix. As explained above, the nomogram curve (security boundary) is given by an equation $B_{d}(D)=0$. The nomogram curve has a normal vector given by the vector $N$. Vector $N$ has two forms expressed in the parameter space, $N_{p}$, controlled parameter space, $N_{c}$, or descriptor parameter space, $N_{d}$.

The nomogram curve $B_{d}(D)=0$ immediately induces a corresponding hypersurface in the parameter space defined by the equation

$$
B_{d}\left(D_{0}+D P^{t} \cdot P\right)=0
$$

Differentiating $B_{d}\left(D_{0}+D P^{t} \cdot P\right)$ with respect to $P$ gives the normal vector to the parameter space hypersurface

$$
N_{p}=N_{d} \cdot D P^{t}
$$

This formula expresses the parameter space normal $N_{p}$ in terms of the nomogram curve normal $N_{d}$ and the transformation matrix $D P^{t}$.

Now we express the nomogram curve normal in terms of the parameter space normal.

Assume a choice of $\Delta P_{1}$ and $\Delta P_{2}$ so that

$$
P=P_{0}+\left(\Delta P_{1}, \Delta P_{2}\right) \cdot M^{-1} \cdot\left(D-D_{0}\right)
$$

Let the parameter space hypersurface be given by an equation

$$
B_{p}(P)=0
$$


The hypersurface has normal vector given by the vector $N_{p}$. Then the nomogram curve is given by

$$
B_{p}\left[P_{0}+\left(\Delta P_{1}, \Delta P_{2}\right) \cdot M^{-1} \cdot\left(D-D_{0}\right)\right]=0
$$

Differentiating the left-hand side with respect to $D$ gives the normal vector to the nomogram curve

$$
N_{d}=N_{p}^{t} \cdot\left(\Delta P_{1}, \Delta P_{2}\right) \cdot M^{-1}
$$

This formula expresses the nomogram's normal vector in terms of the normal vector in the parameter space and a linear transformation.

\subsection{Finding Hyperplanes, Their Normal Vectors, and Sensitivities}

In the descriptor space, the security boundary $B_{d}$ that separates secure system conditions from insecure system conditions is a nonlinear multidimensional manifold, with the dimension $n_{d}-1$, where $n_{d}$ is the full problem dimension. Each manifold, corresponding to a specific system security limit in single contingency, can be approximated by one or several hyperplanes - see Figure 2.1 .

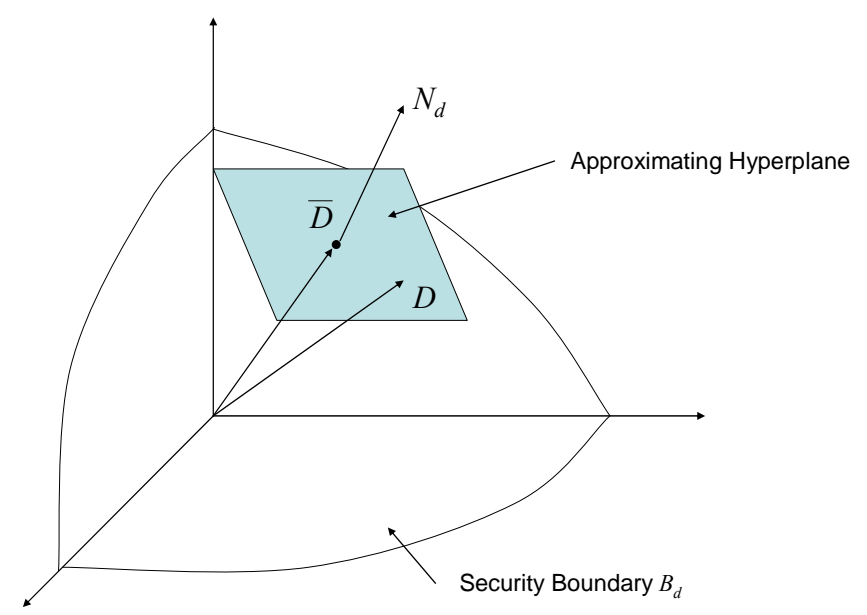

Figure 2.1. Illustration of an Approximating Hyperplane in the Descriptor Parameter Space

\subsubsection{Tangent Hyperplane Definition Using Normal Vector}

A tangent hyperplane, $H_{d}$, touches the boundary, $B_{d}$, in at least one point $\bar{D}$ and never intersects it. ${ }^{6}$ To define the tangent hyperplane, for example, in the descriptor space, it is sufficient to find a point $\bar{D}$ on the security boundary, and then find a normal vector $N_{d}$ to the hyperplane at this point. Having done that, the hyperplane can be described using the following equations:

\footnotetext{
${ }^{6}$ Our assumption at this moment is that the security boundary is convex.
} 


$$
N_{d}^{t} \cdot(D-\bar{D})<0
$$

or

$$
N_{d}^{t} \cdot D<L_{d}
$$

where $D$ is a point in the descriptor space, $N_{d}=\left[\begin{array}{llll}\eta_{d, 1}, & \eta_{d, 2}, & \cdots & \eta_{d, n_{d}}\end{array}\right]^{t}$ is the normal vector and $L_{d}=N_{d}^{t} \cdot \bar{D}$ is a constant. Vector $N_{d}$ is a vector directed outside the security region, as shown in Figure 2.1. Although vector $N_{d}$ can be calculated directly and analytically using some existing methods and for some types of security limits, its direct evaluation remains a computational challenge in general case.

\subsubsection{Secant Hyperplane Definition Using $n$ Point Approach}

In the proposed methodology, we suggest approximating hyperplanes to be evaluated using at least $n_{d}$ pre-calculated points of the security boundary. The hyperplanes evaluated by this approach actually become secant planes. The condition is that these $n_{d}$ points span an $\left(n_{d}-1\right)$-dimensional subspace in $\square^{n}$.

If $n_{d}$ points $\bar{D}_{1}, \bar{D}_{2}, \ldots, \bar{D}_{n_{d}}$ span a hyperplane, then

$$
\begin{gathered}
N_{d}^{t} \cdot \bar{D}_{i}-L_{d}=0 i=1,2, \cdots, n_{d} \\
\eta_{d, 1}=1
\end{gathered}
$$

Without loss of generality, the first element $\eta_{d, 1}$ of the normal vector $\eta_{d, 1}$ can be set as 1 . Equation (2.18) can be rewritten as

$$
A \cdot x=y
$$

where $x=\left[\begin{array}{lllll}\eta_{d, 1}, & \eta_{d, 2}, & \cdots & \eta_{d, n}, & L_{d}\end{array}\right]^{t}, y=\left[\begin{array}{lllll}0, & 0, & \cdots & 0, & 1\end{array}\right]^{t}, x, y \in \square^{n+1}$,

$$
\begin{aligned}
& A=\left[\begin{array}{ccc}
\leftarrow \bar{D}_{1}^{t} \rightarrow & \uparrow \\
\leftarrow \bar{D}_{2}^{t} \rightarrow & \mid \\
\vdots & s \\
& \mid \\
& \bar{D}_{n}^{t} \rightarrow & \downarrow \\
\leftarrow-z-\rightarrow &
\end{array}\right], s=\left[\begin{array}{lllllll}
-1, & -1, & \cdots & -1, & -1
\end{array}\right]^{t}, \text { and } z=\left[\begin{array}{lllll}
1, & 0, & \cdots & 0, & 0
\end{array}\right], \\
& s \in \square^{n}, z \in \square^{n+1} .
\end{aligned}
$$

A solution of Equation (2.19) can be obtained as follows 


$$
x=A^{-1} \cdot y
$$

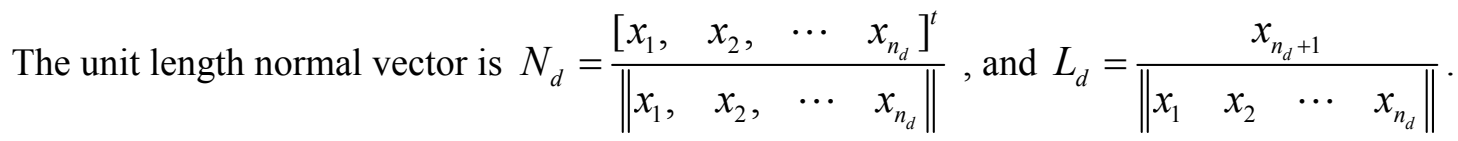

After the normal vector $N_{d}$, which has $\left\|N_{d}\right\|=1$, is determined, the shortest distance $\xi_{d}$ from the current operating point $D_{0}$ to the hyperplane $H_{d}$ is equal to the length of the projection of a vector $D_{0}-\bar{D}$ connecting the current operating point $D_{0}$ to the boundary point $\bar{D}$ onto $N_{d}$ :

$$
\xi_{d}=N_{d}^{t} \cdot\left(D_{0}-\bar{D}\right)
$$

$\xi_{d}$ is the measure of the system security margin.

\subsubsection{Finding Secant Hyperplanes with Specified Security Margin [12]}

Tangent hyperplanes obtained with the help of their normal vectors, as described in Section 2.2.1, can give an overestimated "external" security region description. To avoid possible violations of system security limits because of the approximation errors, the security region should be approximated using "internal" secant hyperplanes. These hyperplanes can be obtained by parallel shifting tangent hyperplanes inside the security region, in the direction of vector $-N_{d}$, by certain configurable value $\delta_{d}$ (in the case if this shifting is needed at all). Additionally, the user may specify certain additional security margin $\mu_{d}$ that must be added to $\delta_{p}$. Therefore, the condition is that the internal hyperplanes should not deviate from the actual boundary for more than $\delta_{d}+\mu_{d}$, and should not be closer to the boundary than $\mu_{d}$. Figure 2.2 gives the idea of such an internal approximation.

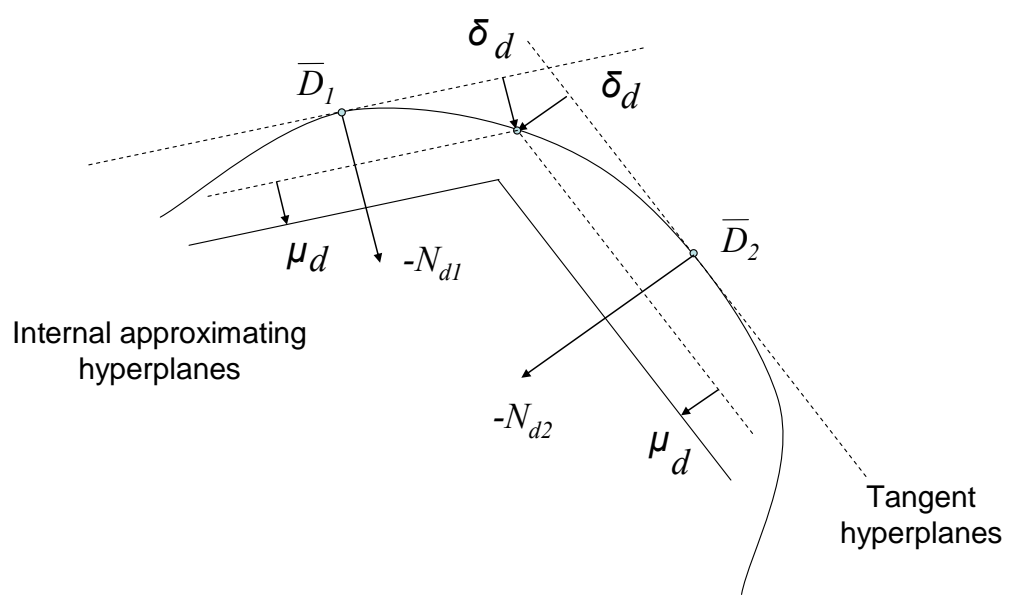

Figure 2.2. Internal Approximation of the Security Region in the Space of Descriptor Parameters 
The condition for an $i$-th internal hyperplane becomes:

$$
N_{d, i}^{t} \cdot\left(D_{i}-\bar{D}_{i}\right)+\delta_{d}+\mu_{d} \leq 0
$$

For the secant hyperplanes obtained using the $n$-point approach described in Section 2.2.2, the "inside" shift $\delta$ is not required, so that $\delta=0$.

\subsubsection{Producing Linear Inequalities}

For a hyperplane $i$ condition, Equation (2.22) can be rewritten as follows:

$$
\begin{gathered}
N_{d, i}=\left[\begin{array}{c}
\eta_{d, i, 1} \\
\eta_{d, i, 2} \\
\ldots \\
\eta_{d, i, n_{p}}
\end{array}\right], \quad D=\left[\begin{array}{c}
d_{1} \\
d_{2} \\
\ldots \\
d_{n_{d}}
\end{array}\right], \quad \bar{P}_{i}=\left[\begin{array}{c}
\bar{d}_{1, i} \\
\bar{d}_{2, i} \\
\ldots \\
\bar{d}_{n_{d}, i}
\end{array}\right] \\
N_{d, i}^{t} \cdot D=\eta_{d, i, 1} \cdot d_{1}+\eta_{d, i, 2} \cdot d_{1}+\ldots+\eta_{d, i, n_{d}} \cdot d_{n_{d}} \leq \underbrace{N_{d, i}^{t} \cdot \bar{D}_{i}}_{L_{d, i}}-\delta_{d}-\mu_{d}
\end{gathered}
$$

The last condition produces one of the approximating linear inequalities.

\subsubsection{Procedure to Calculate Sensitivities of the Security Boundary Points}

The security region can be defined in the space of descriptor (= critical) parameters $D$ (Figure 2.3).

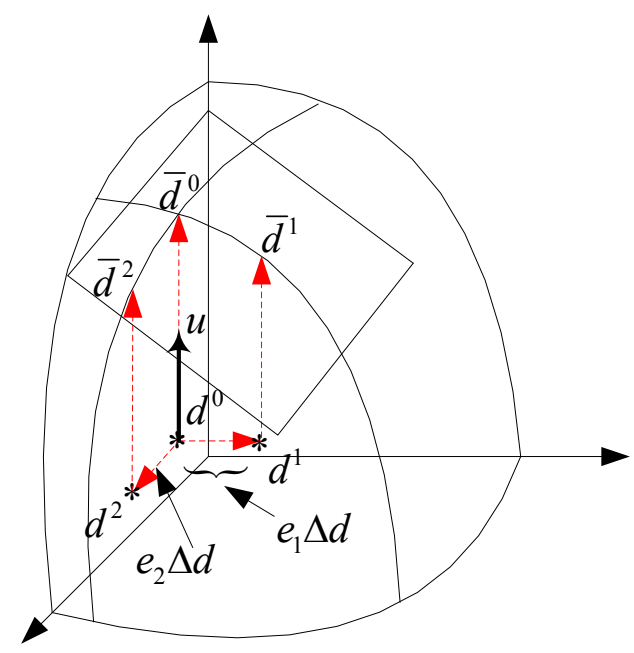

Figure 2.3. Illustration of Security Boundary in the Descriptor Space 
Assume that the descriptor parameters are denoted as

$$
D=\left[\begin{array}{llll}
d_{1}, & d_{2}, & \cdots & d_{n_{d}}
\end{array}\right]^{t}
$$

where $n_{d}$ is the number of these parameters.

The following procedure describes steps to determine sensitivities at the points along the security boundary.

1. Assume that the original state is represented by $D_{0}$,

$$
D_{0}=\left[\begin{array}{llll}
d_{0,1}, & d_{0,2}, & \cdots & d_{0, n_{d}}
\end{array}\right]^{t}
$$

Let the descriptor parameters $d_{i}$ being changed in a stress direction specified using a unit vector $\Delta D$ by varying a scalar continuation parameter $\alpha$. The procedure is stopped when it reaches a security boundary point, which is represented by

$$
\bar{D}_{0}=D_{0}+\overline{\alpha_{0}} \cdot \Delta D
$$

2. At the $i$-th step, $i=1,2, \ldots, n_{d}$, we apply a small increment $e_{i} \cdot \delta d$

$$
\left(e_{i}=\left[\begin{array}{llcll}
0 & \cdots & 1 & \cdots & 0
\end{array}\right]^{t}\right) \text { to vector } D_{0} \text {, and find a new state } D_{i} \text {, where } D_{i}=D_{0}+e_{i} \cdot \delta d \text {. }
$$

When we stress the system from this new point $D_{i}$ to the security boundary

$$
\overline{D_{i}}=D_{i}+\bar{\alpha}_{i} \cdot \Delta D
$$

3. The sensitivity vector is calculated as

$$
S_{i}=\frac{\bar{D}_{i}-\bar{D}_{0}}{\delta d}
$$

\subsection{Building the Security Region in a Multi-dimension Parameter Space}

It is assumed that the procedure is performed in the space of $n_{d}$ parameters (descriptor parameters), $D=\left[d_{1}, d_{2}, \ldots, d_{n_{d}}\right]$.

An approximating hyperplane in the $n_{d}$-dimensional space is determined by $n_{d}$ points on the security boundary, $B_{d}$, which should be located in a physically viable region in the descriptor parameter space. Points on the security boundary are found by changing descriptor parameters until one of the following constraints are violated: thermal, voltage, voltage stability, transient stability and oscillatory/small signal stability. There are two essential elements: a starting point (or a base point), $D_{0}$, and a stress direction, $\Delta D$ in the searching process of boundary points, $\bar{D}$, that determine which part of the security boundary 
will be approximated by a hyperplane $H_{d}$. Contingencies are applied when moving along the stress direction.

\subsubsection{Basic steps}

Trade-off should be made between the number of hyperplanes and the accuracy of using hyperplanes to approximate the nonlinear security region. Large errors can lead to misjudgment on the security condition of the system. The approximation error margin chosen in this project is $50 \mathrm{MW}$ in addition to the security margin ( $5 \%$ for $\mathrm{N}-1$ contingency and $2.5 \%$ for $\mathrm{N}-2$ contingency) ${ }^{7}$.

The flow chart of the simulation steps is shown in Figure 2.4. It consists of four steps:

1. Load WECC data;

2. Identify area of interest in the multi-dimension parameter space;

3. Build the initial hyper-plane and

4. Building refining hyper-planes.

It is an iterative process, and it completes when the pre-defined acceptable error threshold (50 MW) has been met. This methodology is very simple but effective. Each step will be discussed in the following sub-sections.

\footnotetext{
${ }^{7}$ Different error margin can be selected, and the methodology illustrated here can be generally applied.
} 


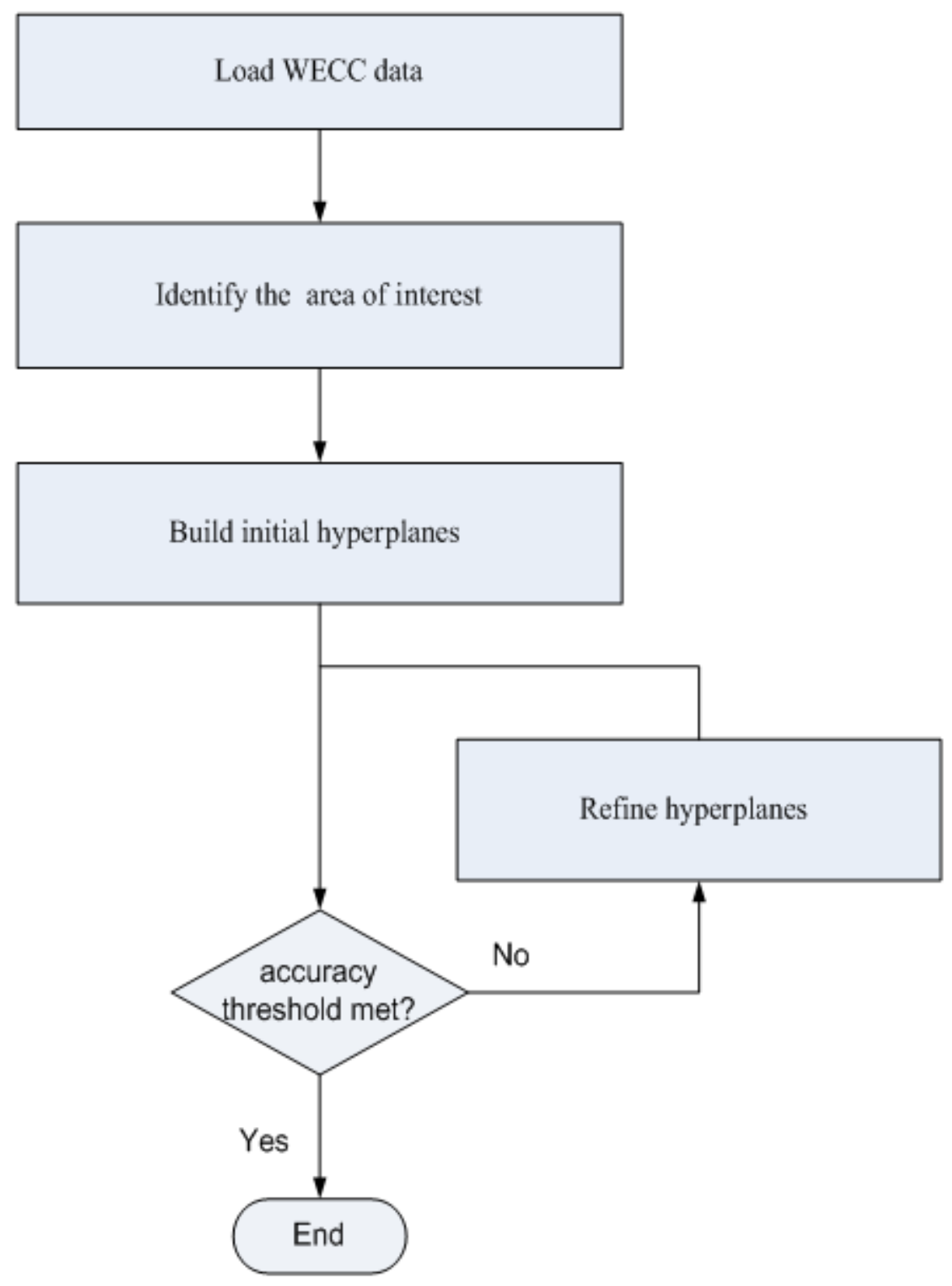

Figure 2.4. Flow Chart of Simulation

\subsubsection{Determine the Region of Interest in the Description, Control, and Independent System Parameter Spaces}

The physically viable region in the parameter space can be derived from the system physical limits such as maximum/minimum generation or load or from the experience. For instance, system constraints can be represented as follows: 


$$
\begin{aligned}
& d_{i} \geq d_{i, \max } \\
& c_{i, \min } \leq c_{i} \leq c_{i, \max } \\
& p_{i, \min } \leq p_{i} \leq p_{i, \max }
\end{aligned}
$$

Figure 2.5 shows an example of a nonlinear security boundary $B_{d}$ in the physically viable region in a three-dimensional descriptor space. From the knowledge of system parameters, one assumes that descriptor variables $d_{1}$ and $d_{2}$ cannot be negative. Therefore, only the limits in the positive directions of $d_{1}$ and $d_{2}$ need to be explored.

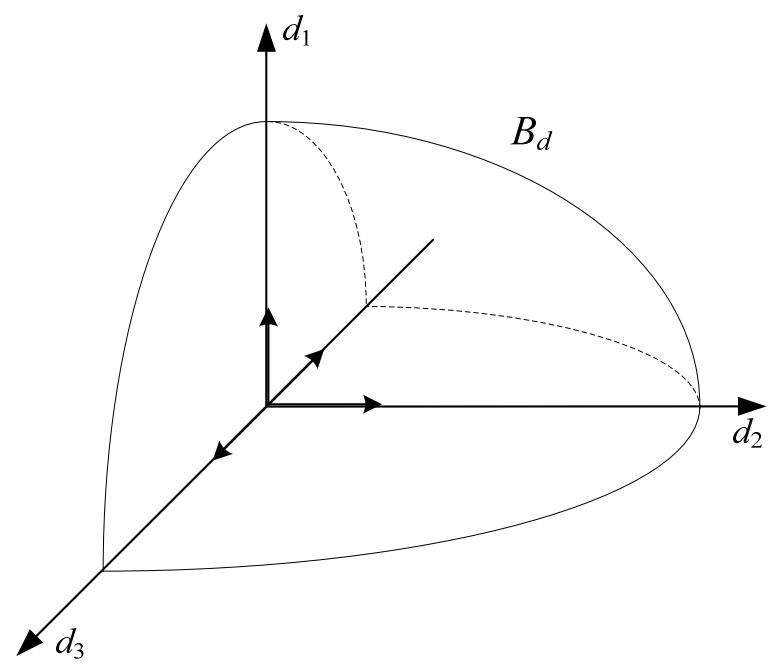

Figure 2.5. Security Boundary in the Area of Interest in a Three-dimensional Descriptor Space

The feasible region, which is denoted as the region of interest, represents where the hyperplanes need to cover. Outside of this region is not of importance to operators because the system will only operate within the regions under the normal conditions.

In this study (2007 heavy summer case), flows $d_{1}, d_{4}, d_{5}, d_{6}, d_{7}$, and $d_{8}$ are assumed to only transfer power from north to south, while power flows along $d_{2}$ and $d_{3}$ are bi-directional (from west to east and from east to west). The region of interest is summarized in the following table. 
Table 2-1. Region of Interest ${ }^{8,9}$

\begin{tabular}{||l|c|c|c|c|c|c|c|c||}
\hline \hline & $d_{1}$ & $d_{4}$ & $d_{5}$ & $d_{6}$ & $d_{7}$ & $d_{8}$ & $d_{2}$ & $d_{3}$ \\
\hline 1 & - & + & + & + & + & + & + & + \\
\hline 2 & - & + & + & + & + & + & + & - \\
\hline 3 & - & + & + & + & + & + & - & + \\
\hline 4 & - & + & + & + & + & + & + & - \\
\hline
\end{tabular}

\subsubsection{Starting Point and Stress Directions to Find Initial Hyperplanes}

The initial set of hyperplanes should be able to cover as much of the security region as possible with a minimum number of hyperplanes. When building initial hyperplanes $H_{d, i}, i=1,2, \ldots$, the boundary points used to determine the hyperplane should be well distributed over the viable part of the descriptor space, so that the region of interest can be covered by the hyperplanes' set.

The security region boundary is a complete set of all the boundary points. A procedure has been designed to approximate the security region boundary with satisfactory accuracy, which begins with finding initial hyperplanes and refine them with more hyperplanes if needed to improve accuracy in approximation. It is very easy to implement and effective to extend the security region to the whole space with the minimum number of hyperplanes. The main steps are described in as follows, illustrated in Figure 2.6 and Figure 2.7.

1. Find a base point (a solved power flow case) located in the region under study, shown as $D_{0}$ in Figure 2.6.

2. Stress the system in multiple directions $\Delta D_{1}, \Delta D_{2}, \Delta D_{3}, \ldots, \Delta D_{k}, k \geq n_{d}$ covering the viable region and find the corresponding security boundary points. In Figure 2.6, six stress directions have been selected (shown as blue arrows), and six boundary points have been found: $1,2,3,1^{\prime}, 2^{\prime}$, and $3^{\prime}$.

3. Build the initial hyperplanes using the boundary points. Each hyperplane requires $n_{d}$ neighboring points. The points are treated as neighboring points if the stress directions, which generates these points, form a sector, where none of the other stress direction can be found. For instance, in Figure 2.6, points 1,2, and 3 form a hyperplane (because there is no more stress direction found in the sector, formed by $\Delta D_{1}, \Delta D_{2}$ and $\Delta D_{3}$, while points $\Delta D_{1}, \Delta D_{2}$, and $\Delta D_{2}$, do not form a hyperplane (because, for instance, $\Delta D_{1}$ belong to the sector, formed by $\Delta D_{2}$, and $\Delta D_{2^{\prime}}$ ). In Figure 2.6, eight hyperplanes are built:

\footnotetext{
${ }^{8}+$ means positive value and - means negative value

${ }^{9}$ Positive and negative direction for $d_{1}$ are from south to north and from north to south, respectively;

Positive and negative direction for $d_{4}, d_{5}, d_{6}, d_{7}$, and $d_{8}$ are from north to south and from south to north, respectively;

Positive and negative direction for $d_{2}$ and $d_{3}$ are from east to west, and from west to east respectively.
} 
$H_{d, 1}$ formed by the boundary points $1,2,3$;

$H_{d, 2}$ formed by the boundary points $1,2,3$;

$H_{d, 3}$ formed by the boundary points $1,2^{\prime}, 3^{\prime}$;

$H_{d, 4}$ formed by the boundary points $1,2^{\prime}, 3$;

$H_{d, 5}$ formed by the boundary points $1,2,3$;

$H_{d, 6}$ formed by the boundary points $1^{\prime}, 2,3^{\prime}$;

$H_{d, 7}$ formed by the boundary points $1,22^{\prime}, 3^{\prime}$, and

$H_{d, 8}$ formed by the boundary points $1^{\prime}, 2^{\prime}, 3$

4. Add the initial hyperplane to the developing set.

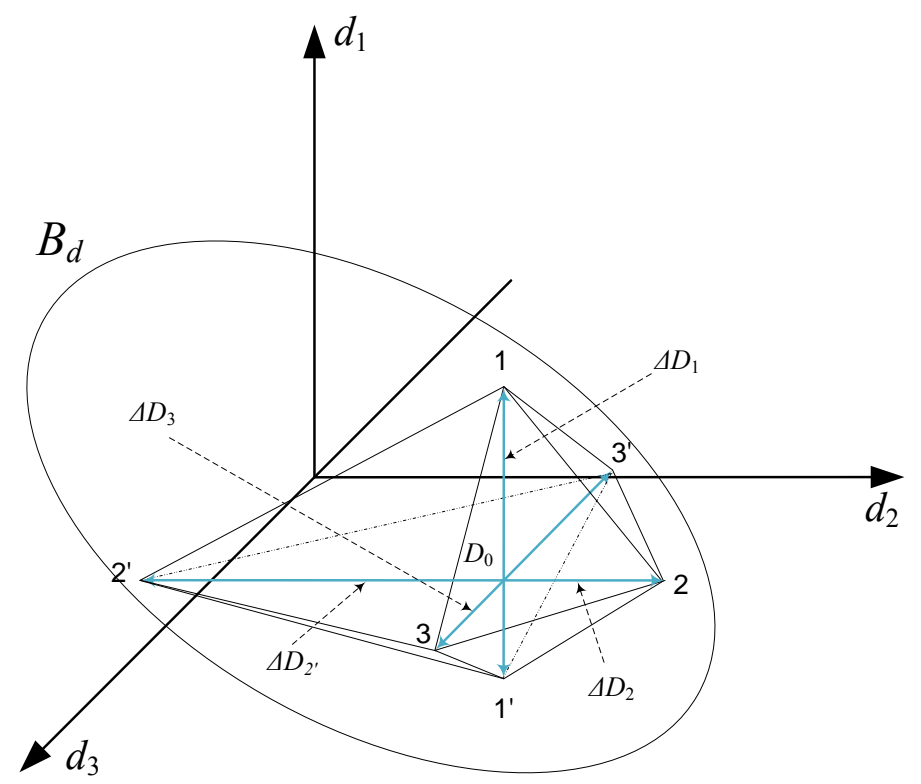

Figure 2.6. Stressing the System to Find Initial Hyperplanes

\subsubsection{Developing the Set of Hyperplanes Based on a Given Accuracy of Approximation}

More hyperplanes should be built to achieve a given accuracy of approximation if needed. The algorithm for this procedure is explained as follows.

1. Select a hyperplane from the developing set, $H_{d, i}$.

2. Find a center of gravity of a multidimensional polyhedron that forms the hyperplane. If the vertices of the polyhedron are assigned equal weights, the center of gravity is the same as the geometric center of the polyhedron, which is the average of the coordinates of all vertices. Define a stress direction, which connects the base point $D_{0}$ with the center of gravity. For instance, in Figure 2.7, stress direction $\Delta D_{4}$ connects $D_{0}$ with the center of the two-dimensional polyhedron $1,2^{\prime}, 3^{\prime}$ that defined hyperplane $H_{d, 3}$. 


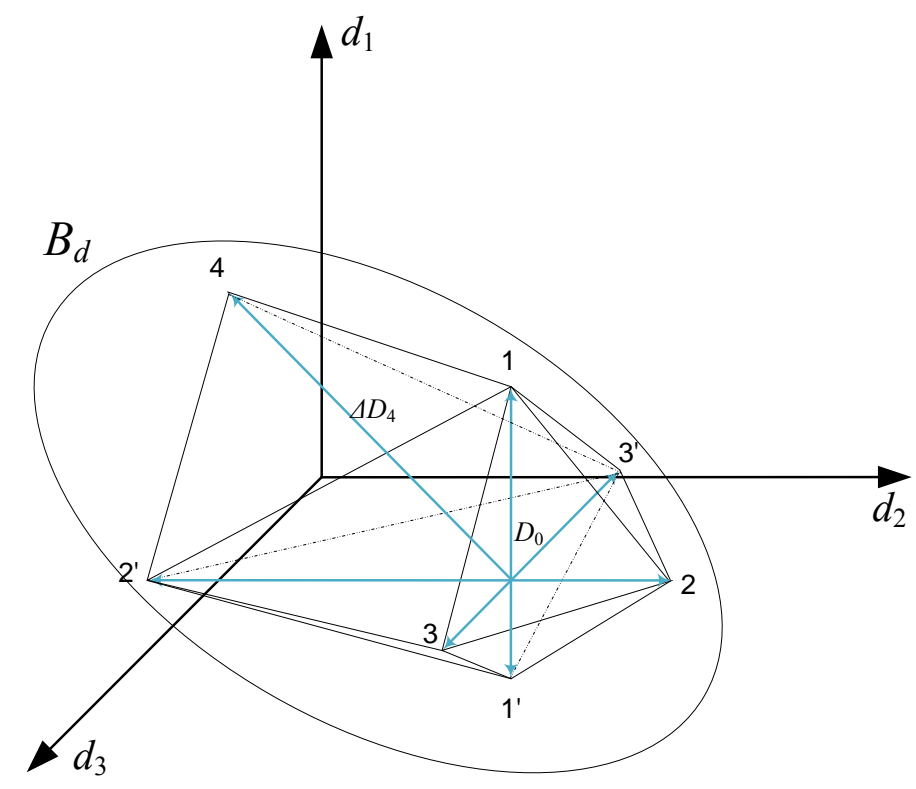

Figure 2.7. Developing the Hyperplanes' Set

3. Find a boundary point in this stress direction (e.g., point 4 in Figure 2.7). Please note that the viable region constraints (Equation (2.30)) should be ignored at this step. Check the distance from the center of gravity to the new point. If the distance does not exceed a given maximum $\delta_{d}$ (which means the hyperplane is close enough to the actual boundary), eliminate hyperplane $H_{d, i}$ from the developing list and memorize it in the list of final hyperplanes.

4. Otherwise, form $n_{d}$ new hyperplanes using $\left(n_{d}-1\right)$ points, forming the polyhedron for $H_{d, i}$ and add them to the developing set. (Note that by adding just one more boundary point, we obtain new $n_{d}$ hyperplanes) Eliminate hyperplane $H_{d, i}$ from the developing set. In our example, these new hyperplanes are spanned by the following polyhedrons: $1-2^{\prime}-4,1-3 '-4$, and $2^{\prime}-3^{\prime}-4$.

5. For each new hyperplane, check if at least one of its polyhedron's points belong to the viable region (Equation (2.30)). If all points are found outside of the viable region, eliminate the corresponding hyperplane from the developing set.

6. Continue this procedure until the developing set is empty. Use the final set of hyperplanes for the security analysis.

\subsubsection{Stressing the System to Find the Security Boundary Points}

In the algorithms given above, to eliminate unnecessary computational work, it is suggested to check all types of constraints for all analyzed contingencies while incrementing system parameters along a given stress direction. The following algorithm reflects this idea. 
1. Set a sequence to check different types of limits at each incremental step: steady-state limits (thermal, voltage limits, voltage stability) and transient-state limits (transient stability and small signal stability).

2. Stress the system along the determined stress direction from a base point.

3. Start from the first contingency in the provided list until the last one is reached to check if any contingency causes violations of steady-state limits. If not, move to the transient-state limits. If none of the limits are violated by any contingencies, use the current point as the new base point and go back to Step 2;

4. If any violation occurs during Step 3, record the current point as a security boundary point.

To save simulation efforts, BPA often considers the most limiting constraints (known from experience) before the others. For example, voltage stability limit is the most limiting factor for $d_{4}$; therefore, it is checked before transient limits to reduce simulation efforts. A detailed example is shown in Section 3.3.

\subsection{Security Region Description and Security Margin}

This section gives the mathematical formulation of security region and security margin.

\subsubsection{Security Region Description Using a Set of Linear Inequalities}

The approximating security conditions are calculated offline as a set of inequalities specific for each analyzed contingency and type of security violation:

$$
\left\{\begin{array}{l}
\eta_{d, 1,1} d_{1}+\ldots+\eta_{d, 1, n} d_{n_{d}} \leq L_{d, 1} \\
\eta_{d, 2,1} d_{1}+\ldots+\eta_{d, 2, n} d_{n_{d}} \leq L_{d, 2} \\
\ldots \\
\eta_{d, m, 1} d_{1}+\ldots+\eta_{d, m, n} d_{n_{d}} \leq L_{d, m}
\end{array}\right.
$$

The number of constraints is $m$, and the number of descriptor parameters included in each constraint is $n_{d}$. Figure 2.8 gives a conceptual example of the approximated security region, represented as multidimensional polyhedron. Each face of the region approximates a part of the nonlinear region's boundary. The approximated region is determined as the inner intersection of constraints for various types of violations and analyzed contingencies.

\subsubsection{Advantages of the proposed approach}

- Fast and convenient assessment of the system security conditions. Having constraints (A.17) precalculated offline for each analyzed contingency, it is very easy to quickly determine in real time:

- Whether the operating point $D_{0}$ is inside or outside the security region (by making sure that all approximating inequalities are satisfied),

- Which constraints are violated (by identifying violated inequalities), and 
- What the most limiting constraints are (by calculating the distance from the current operating point $D_{0}$ to the approximating planes $H_{d}$, as described below).

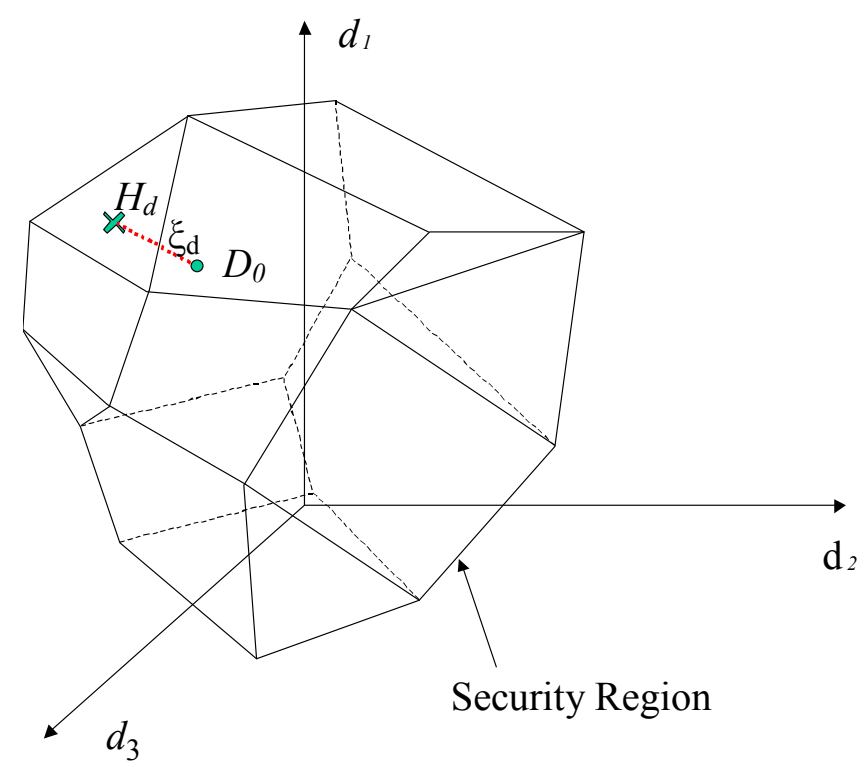

Figure 2.8. Conceptual View of the Approximated Security Region

- Easy-to-calculate security margin. The distance $\xi_{d, i}$ from the current operating point $D_{0}$ to the nearest constraint hyperplane $H_{d, i}$ determines the MW security margin: ${ }^{10}$

$$
\xi_{d, i}=\frac{\left|\eta_{d, i 1,} d_{1}^{0}+\ldots+\eta_{d, i, n_{d}} d_{n_{d}}^{0}-L_{d, i}\right|}{\left\|N_{d, i}\right\|}
$$

where the current operating point $D_{0}=\left[d_{1}^{0}, \ldots, d_{n_{d}}^{0}\right]$

The resulting stability margin corresponding to the minimum distance from the stability boundary, i.e., the distance to the closest constraint face, must be greater than certain minimum security margin $\mu_{d}:$

$$
\min _{(i)} \xi_{d, i} \geq \mu_{d}
$$

\subsection{Most Effective Controls, Weak Elements, and Remedial Actions}

This section discusses the approach to find the most effective control directions to increase security margin, identify the element that is constraining system transmission capability and suggest remedial actions.

\footnotetext{
${ }^{10}$ We assume that the region is convex.
} 


\subsubsection{Critical and Subcritical Distances and Compromise Control}

In descriptor parameter space, assume that the security region consists of a set of $m$ hyperplanes:

$$
H_{d, i}: \quad \eta_{d, i, 1} D_{1}+\eta_{d, i, 2} D_{2}+\cdots+\eta_{d, i, n_{d}} D_{n_{d}} \leq L_{d, i} \quad i=1, \cdots, m
$$

where $n_{d}$ is the dimension of the considered space, and $N_{d, i}=\left[\eta_{d, i, 1}, \eta_{d, i, 2}, \cdots, \eta_{d, i, n_{d}}\right]^{t}$ is the normal vector of the hyperplane $H_{d, i}$. We can determine an operating point $D_{0}=\left[d_{0,1}, d_{0,2}, \cdots, d_{0, n_{d}}\right]$ from PMU (Phasor measurement unit) data, SCADA data, and state estimation. Then we can compute distances from $D_{0}$ to hyperplanes $H_{d, i}, \quad i=1, \cdots, m$,

$$
\xi_{d, i}=\frac{\left|\eta_{d, i 1}, d_{1}^{0}+\ldots+\eta_{d, i, n_{d}} d_{n_{d}}^{0}-L_{d, i}\right|}{\left\|N_{d, i}\right\|}, i=1, \cdots, n
$$

Let us renumber $\xi_{d, i}, i=1, \cdots, m$ so that $\xi_{d, 1} \leq \xi_{d, 2} \leq \cdots \leq \xi_{d, m}$ and normalize $\xi_{d, i}$ to the minimum security margin $\mu_{d}$ :

$$
\tilde{\xi}_{d, i}=\frac{\xi_{d, i}}{\mu_{d}}, \quad i=1, \cdots, m
$$

Now we can divide $\tilde{\xi}_{d, i}, i=1, \cdots, m$ into two groups (critical and non-critical) based on their normalized values, for instance

a. Critical group: $\tilde{\xi}_{d, i} \leq 1$, and

b. Non-critical group: $\tilde{\xi}_{d, i}>1$.

The critical hyperplane $H_{d, 1}$ corresponds to $\tilde{\xi}_{d, 1}$. But the rest of the distances in the critical group with $\tilde{\xi}_{d, i} \leq 1$ (subcritical distances) are also considered as contributing to the insufficient security margin, and the most effective direction to control the system can be found in the direction determined by the compromise control vector $C_{d}$ :

$$
C_{d}=-\sum_{i=1}^{k} \frac{1}{\xi_{d, i}} \frac{N_{d, i}}{\left\|N_{d, i}\right\|}
$$

where $k$ is the number of elements in the critical group. Weight coefficients $\frac{1}{\xi_{d, i}}$ indicate the contribution of the corresponding component in the vector sum (Equation (2.37)), in which the distances to the approximating hyperplanes are minimal. 


\subsubsection{An Illustration of the Critical and Subcritical Distances and Compromise Control}

Assume that in two-dimensional descriptor space the security region is approximated by four hyperplanes, $H_{d, 1}, H_{d, 2}, H_{d, 3}$, and $H_{d, 4}$, with normal vectors $N_{d, 1}, N_{d, 2}, N_{d, 3}$, and $N_{d, 4}$, respectively. The operating point is determined as $D_{0}$. Distances from $D_{0}$ to the four hyperplanes are $\xi_{d, 1}, \xi_{d, 2}, \xi_{d, 3}$, and $\xi_{d, 4}$, respectively - see Figure 2.9. It can be seen that $\xi_{d, 1} \approx \xi_{d, 2}<\xi_{d, 3}<\xi_{d, 4}$, and $\xi_{d, 1}, \xi_{d, 2}$ form the critical group. The most effective direction to control the system is determined by the compromise vector $C_{p}$, that is a weighted combination of normal vectors of the critical group, $C_{d}=-\sum_{i=1}^{2} \frac{1}{\xi_{d, i}} \frac{N_{d, i}}{\left\|N_{d, i}\right\|}$.

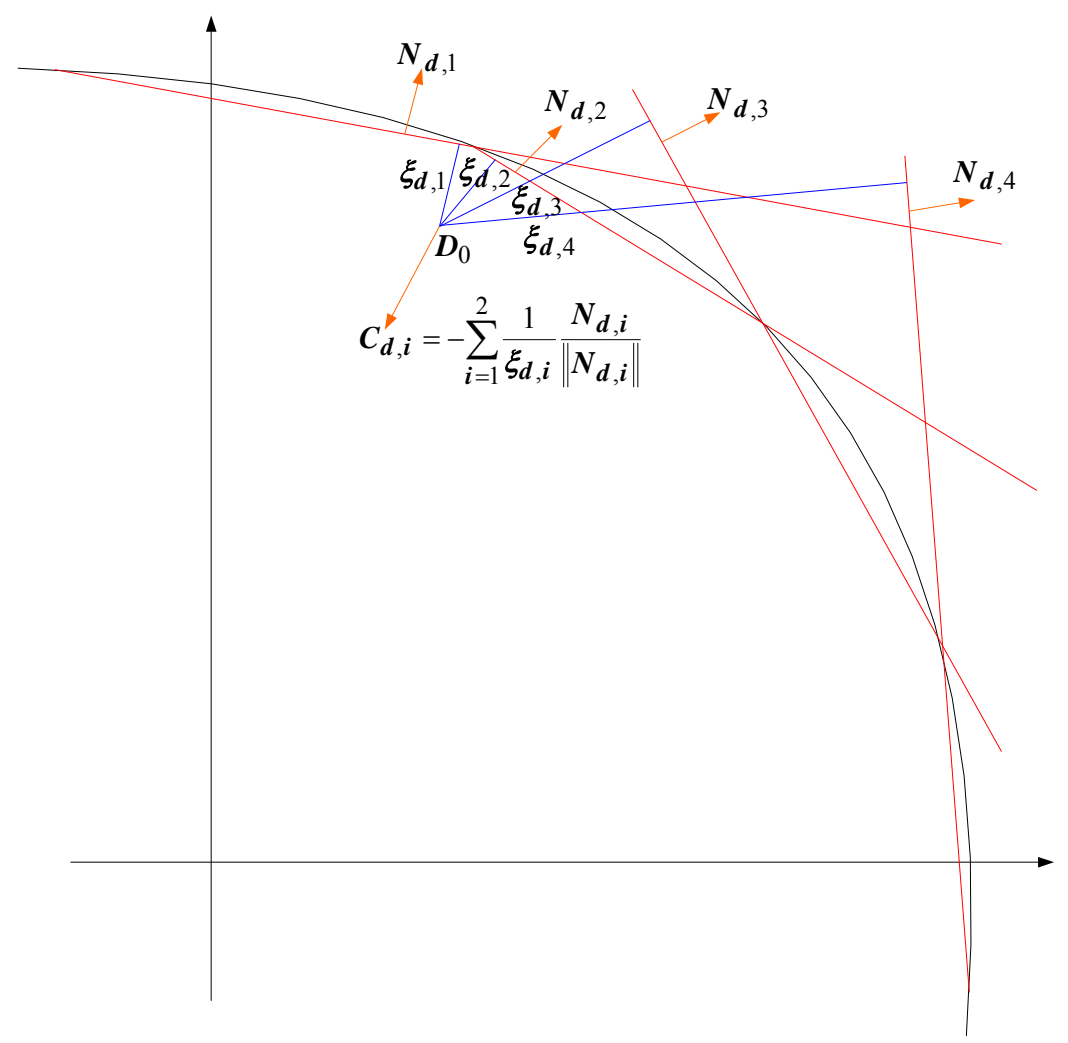

Figure 2.9. An Illustration of the Critical and Subcritical Distances and Compromise Control

\subsubsection{Selecting Controls and Remedial Actions in the Controlled or Independent Parameter Space}

This section discusses about applying the proposed multidimensional security region approach into the dispatch of generation or load. 


\subsubsection{Incorporating Constraints into the Security Constrained Unit Commitment and Dispatch}

Security region boundary can be described using a set of linear inequalities applied to the descriptor parameters $D$ :

$$
\left\{\begin{array}{l}
\eta_{d, 1,1} d_{1}+\ldots+\eta_{d, 1, n} d_{n_{d}} \leq L_{d, 1} \\
\eta_{d, 2,1} d_{1}+\ldots+\eta_{d, 2, n} d_{n_{d}} \leq L_{d, 2} \\
\ldots \\
\eta_{d, m, 1} d_{1}+\ldots+\eta_{d, m, n} d_{n_{d}} \leq L_{d, m}
\end{array}\right.
$$

As described in Section 2.2, limits $L_{d, 2}$ can be modified to incorporate the required security margin $\mu_{d}:$

$$
\left\{\begin{array}{l}
\eta_{d, 1,1} d_{1}+\ldots+\eta_{d, 1, n} d_{n_{d}} \leq L_{d, 1}-\mu_{d} \\
\eta_{d, 2,1} d_{1}+\ldots+\eta_{d, 2, n} d_{n_{d}} \leq L_{d, 2}-\mu_{d} \\
\ldots \\
\eta_{d, m, 1} d_{1}+\ldots+\eta_{d, m, n} d_{n_{d}} \leq L_{d, m}-\mu_{d}
\end{array} \Leftrightarrow N_{d}^{t} \cdot D \leq L_{d}-\mu_{d}\right.
$$

These inequalities can be directly used in the security constrained unit commitment and dispatch programs if $D$ in (Equation (2.39)) is substituted by its expression in coordinates of independent parameters: $D=D_{0}+D P^{t} \cdot P$ - see Section 2.1. System generation can be automatically dispatched in the security constrained unit commitment and dispatch software tools obeying security limits (Equation (2.39)).

\subsubsection{Simplified Procedure Using Control Vector $C_{p}$}

If the option for incorporating linearized multidimensional constraints into the security constrained unit commitment and dispatch is not available, a simplified procedure could be suggested instead.

If the security margin is not sufficient ( $\tilde{\xi}_{d, i}<1$ for some hyperplanes $H_{d, i}-$ see Section 2.5 .1 ), it should be increased by applying controls helping to move the operating point along the vector $C_{d}$, as shown in Figure 2.9. Because the descriptor parameters $D$ cannot be controlled directly, a mapping from the descriptor space into the control or independent parameter spaces is needed. In general, this mapping is not uniquely defined. Nevertheless, it still can be done using the affine models for the control and descriptor parameters, as described in Section 2.1. The control vector in the independent parameter state can be expressed as follows.

$$
C_{p}=C_{d} \cdot D P^{t}
$$

where $D P$ is a matrix of coefficients describing a linear combination of independent system parameters forming descriptor parameters $D: D=D_{0}+D P^{t} \cdot P$. Vector $C_{p}$ can be used to identify the best set of 
controls and remedial actions that could help to increase the security margin in the descriptor parameter space by redispatching the system generators or curtailing the loads.

Several principles should be applied when selecting remedial actions or controls.

1. It is impractical to move multiple generators by a few megawatts or disconnect multiple small loads at the same time. If required, the control should be limited by a few, perhaps, more significant changes. Therefore, only the most influential generators and loads should be involved. The influence of each generator on the security margin can be evaluated by calculating the following metric (Equation (2.41)). The metric is actually the cosine of the angle between vector $C_{p}$ and the corresponding coordinate of the independent parameter space $p_{i}$ :

$$
\gamma_{i}=\frac{C_{p}^{t} \cdot e_{i}}{\left\|C_{p}\right\|}, e_{i}=\left[\begin{array}{llcll}
0 & \cdots & 1 & \cdots & 0
\end{array}\right]^{t}
$$

Maximum $\gamma$-factors correspond to the more influential generators and loads.

2. Available control should be prioritized using both absolute and relative priorities. For example, generation redispatch should be given a higher absolute priority compared to the load curtailment. This means that the load curtailment should be only applied if the available generation cannot be redispatched to alleviate critical security problems. Relative priorities should reflect additional factors such as the cost of generation redispatch on different generators.

3. Balanced controls that do not cause system-wide power mismatches should be given preference. To achieve this objective, the balanced controls can be selected among available controls with the opposite $\gamma$-factors.

4. Applied controls will be changing the position of the current operating point in coordinates of independent parameters $P$ and, consequently, in coordinates of descriptor parameters $D$. For each new position, the security check described above should be repeated to determine if the applied control is sufficient.

\subsubsection{Weak Elements}

The best candidate method for revealing weak elements influencing the system security conditions can be based on the following approach:

For each hyperplane, let us analyze of the direction of its normal vector $N_{d}$ shown in Figure 2.10. This vector can be transformed into the space of independent parameters using expression (Equation (2.42)).

$$
N_{p}=N_{d} \cdot D P^{t}
$$

The closer the direction of $\xi_{d}$ is to some independent coordinates of the power system parameters' space $p_{1}^{0}, \ldots, p_{n_{p}}^{0}$, the more influential these nodal powers are. Figure 2.10 illustrates this idea. In this figure, for the analyzed hyperplane, parameter $p_{1}$ is more influential than $p_{2}$, because by changing 
parameter $p_{1}^{0}$, it is possible to quickly increase or decrease the distance to insecurity. A formal indicator of this is the distance sensitivity to the coordinates of the current operating point, in our example, $\frac{\partial \xi}{\partial p_{1}^{0}}$ is greater than $\frac{\partial \xi}{\partial p_{2}^{0}}$.

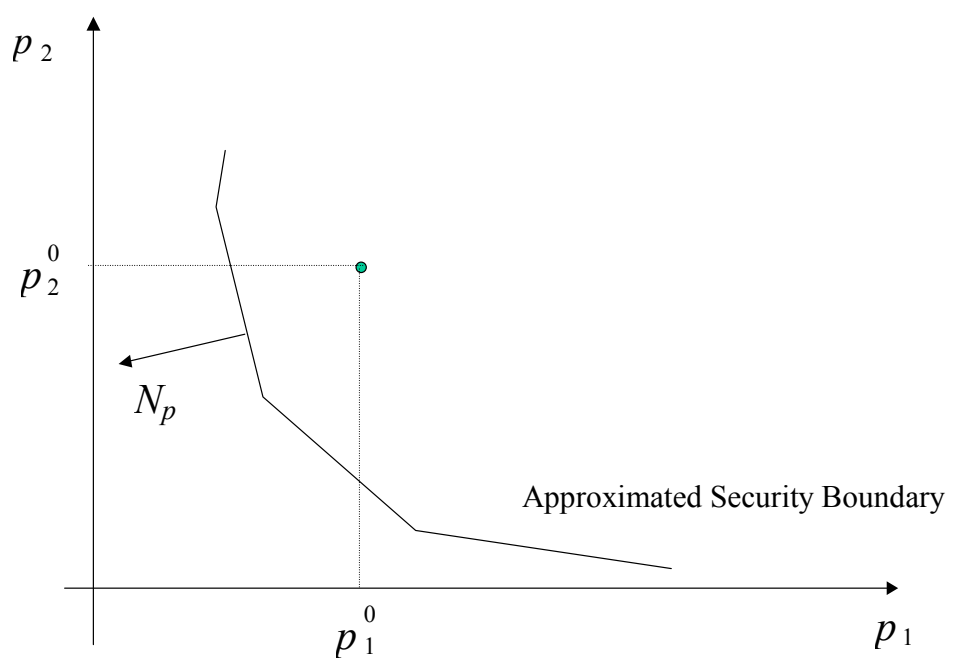

Figure 2.10. Identification of Weak Elements

The mathematical expressions for calculating these sensitivities are very simple:

$$
\frac{\partial \xi_{i}}{\partial p_{j}^{0}}=\frac{\eta_{p, i, j}}{\sqrt{\eta_{p, i, 1}^{2}+\ldots+\eta_{p, i, n_{p}}^{2}}}, i=1, \ldots, m, j=1, \ldots, n_{p}
$$

The power of this approach is that, besides revealing weak elements, it helps to conclude what needs to be done in the system to increase system security margin.

\subsection{Refining Operating Transfer Capability (OTC) Limits Using Multidimensional Nomograms}

This section discusses about the application of multidimensional security region on determining operating transfer capability of a transmission system.

\subsubsection{WECC OTC Provisions}

An actual power flow and net scheduled power flow over a transfer path should be maintained within the operating transfer capability limits (OTC). The OTC is the maximum amount of power that can be transferred over the transmission system comprising:

- An intertie from one balancing authority to another balancing authority; or 
- A transfer path within a balancing authority area.

The net schedule over an interconnection or transfer path within a balancing authority area should not exceed the Operating Transfer Capacity (OTC) limits, regardless of the prevailing actual power flow on the interconnection or transfer path.

a. Operating limits. No elements within the interconnection can be scheduled above continuous operating limits. An element is defined as any generating unit, transmission line, transformer, bus, or piece of electrical equipment involved in the transfer of power within an interconnection.

b. Stability. The interconnected power system shall remain stable upon loss of any one single element without system cascading that could result in the successive loss of additional elements. System voltages shall be within acceptable limits defined in the WECC Reliability Criteria for Transmission System Planning. If a single event could cause loss of multiple elements, these shall be considered in lieu of a single element outage. This could occur in exceptional cases such as two lines on the same right-of-way next to an airport. In either case, loss of either single or multiple elements should not cause uncontrolled, widespread collapse of the interconnected power system. Stability includes transient stability, post transient stability or dynamic stability, whichever is most limiting to OTC.

c. System contingency response. Following an outage and before the outage, adjustments can be made so that:

i. No remaining element shall exceed its short-time emergency rating.

ii. The steady-state system voltages shall be within emergency limits.

The limiting event can be determined by conducting power flow and stability studies while simulating various operating conditions. These studies shall be updated as system configurations introduce significant changes in the interconnection.

Actual power flow on all transmission paths shall at no time exceed the OTC for more than 20 minutes for paths that are stability limited, or for more than 30 minutes for paths that are thermally limited.

\subsubsection{Drawbacks of the Existing Approach to Determine the OTC}

Existing approaches to determine the OTC are based on simplifications of the system model so that the transfer limits could be represented as individual limits specified for specific transmission paths or two- or three-dimensional nomograms. An example of a three-dimensional nomogram is shown in Figure 2.11 .

The existing approach to determine the OTC has to be proven to be a robust way of determining the system security limits. At the same time, there is a question on how conservative this approach is in view of the limited number of dimensions used in the existing nomograms. System descriptor parameters are frequently mutually influential, so that a two or three-dimensional nomogram may not be sufficient to reflect all these interactions. As a result, these nomograms might be made more conservative by simulating the worst case scenarios for the parameters that are not shown in the nomogram. Underestimated security limits lead to unnecessary generation redispatch. 


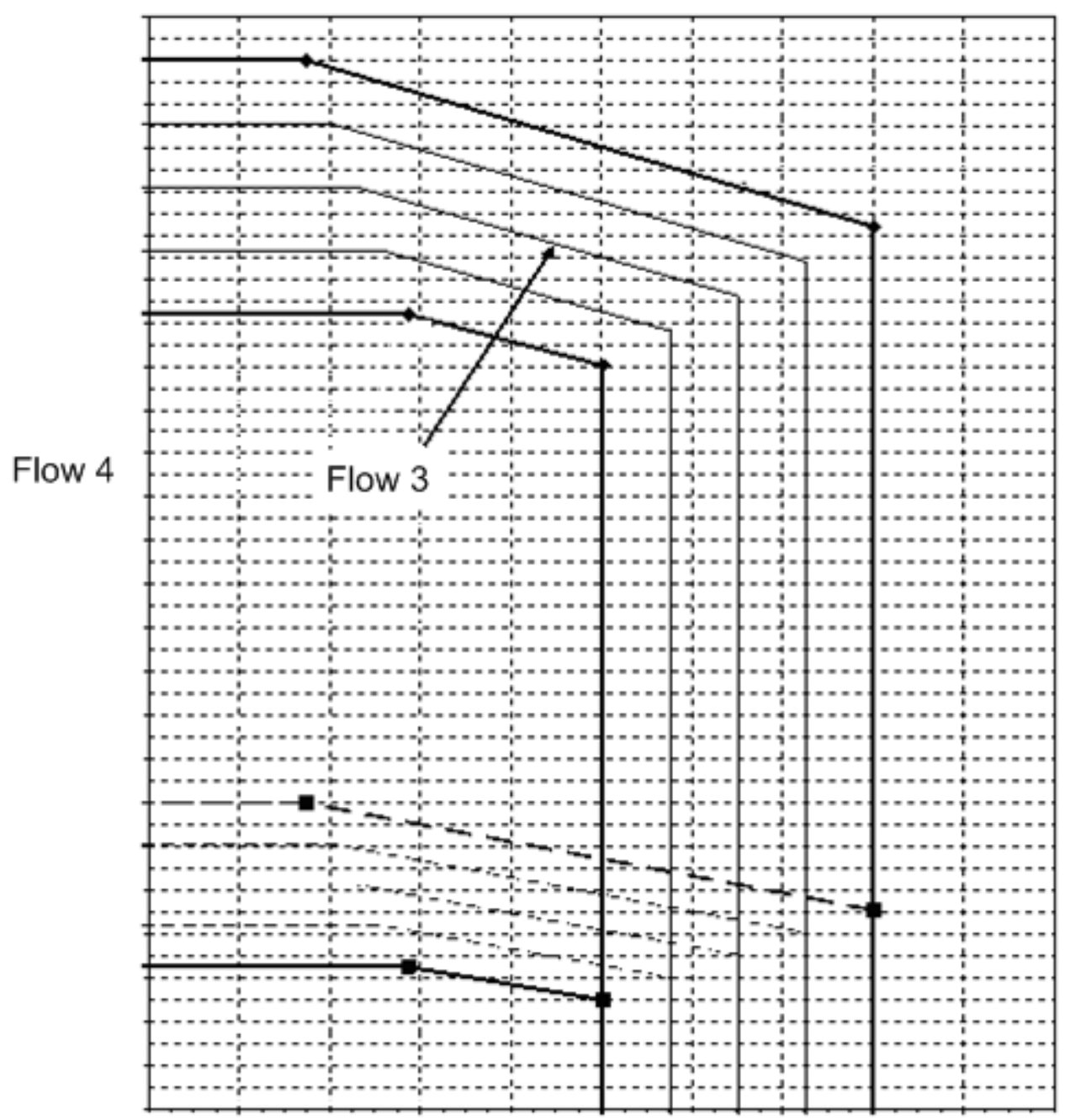

Flow 5

Figure 2.11. Example of three-dimensional Nomogram

\subsubsection{New Approach to Determine OTC Based on $n$-dimensional Nomograms}

The proposed approach could be built around the idea of using the linear inequalities restricting the security region in the multidimensional descriptor parameters space. The set of inequalities will be expressed as follows:

$$
\left\{\begin{array}{l}
\eta_{d, 1,1} d_{1}+\ldots+\eta_{d, 1, n} d_{n_{d}} \leq L_{d, 1}-\mu_{d} \\
\eta_{d, 2,1} d_{1}+\ldots+\eta_{d, 2, n} d_{n_{d}} \leq L_{d, 2}-\mu_{d} \\
\ldots \\
\eta_{d, m, 1} d_{1}+\ldots+\eta_{d, m, n} d_{n_{d}} \leq L_{d, m}-\mu_{d}
\end{array} \Leftrightarrow N_{d}^{t} \cdot D \leq L_{d}-\mu_{d}\right.
$$


The set (2.44) establishes limits on simultaneous power transfers in multiple paths in the system. It helps to find a more flexible, more adaptive, and hopefully less conservative description of the system security limits. It is important to note that the existing definitions for the OTC limits are completely consistent with the proposed approach. For instance, the nomogram shown in Figure 2.11 could be easily represented as five sets of linear inequalities in a four-dimensional descriptor space. 



\subsection{Numerical Results}

The numerical simulation is carried out on the WECC system, which consists of 15,334 buses and 3,034 generators. The WECC 2007 heavy summer case provided by BPA is used as the base case for our study.

\subsection{Descriptor and Control Parameters and Stress Patterns}

Critical parameters that have more impact on system security are often selected for the study to decrease the complexity of the security analysis problem. Based on the engineering experience of BPA's engineers, a subset of the WECC system parameters has been identified to approximate the security boundary of the system and to develop multidimensional nomograms. These parameters are either a combination of controlled parameters (generation or load) or a combination of power flow parameters (line flows).

The critical descriptor parameters and control parameters are selected as follows:

Descriptor Parameters:

1. Flow $1 d_{1}$

2. Flow $2 d_{2}$

3. Flow $3 d_{3}$

4. Flow4 $d_{4}$

5. Flow5 $d_{5}$

6. Flow6 $d_{6}$

7. Flow $7 d_{7}$

8. Flow $8 d_{8}$

Control Parameters:

1. Area1 generation $c_{1}$

2. Area2 generation $c_{2}$

3. Area3 generation $c_{3}$

4. Area4 generation ${ }^{c_{4}}$

5. Area5 generation $c_{5}$ 
6. Area6 load $c_{6}$

7. Arera7 load $c_{7}$

8. Area 8 load $c_{8}$

9. Area9 generation $c_{9}$

Changes of generation and load are always balanced in our studies, that is, changes of the generationload balance in one area are compensated by balance changes in the opposite direction in another area. The resulting changes in power losses are injected (or absorbed) by the swing bus.

It is important to note that the control parameters must be independent, so that combined changes of some of these parameters would not be equivalent to a combined change of the remaining parameters. Selecting dependent parameters for an analysis would result in miscalculation of the approximating hyperplane and its normal vector. This is illustrated by Figure 3.1 for three dimensions. Assuming that $c_{1}$ and $c_{2}$ are interdependently controlled parameters, thus the system security boundary becomes one-dimensional (represented by the red line). Its normal vector is not uniquely determined in the three-dimensional space of $c_{1}, c_{2}$ and $c_{3}$.

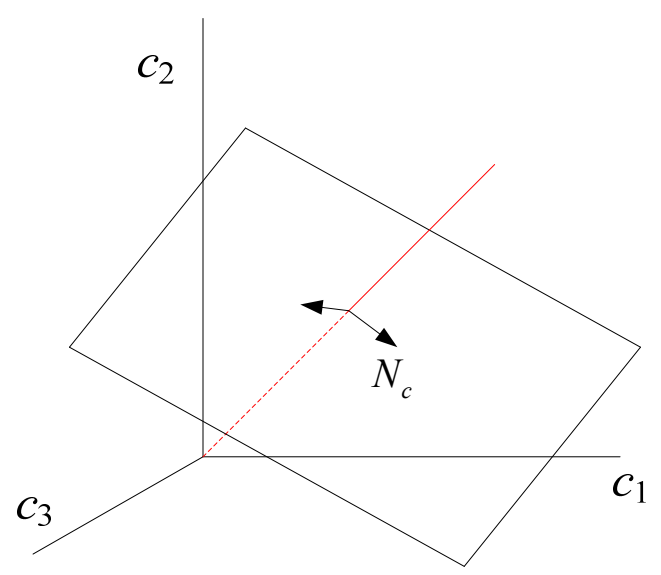

Figure 3.1. Non-uniqueness of the Normal Vector for Interdependent Controlled Parameters

The balanced changes have been applied to the generation and load, as described by the stress patterns. If the summary generation or load in one area is changed, generations or load in some other area will be changed by the same amount, but in the opposite direction. If the power losses are not considered, this results in a zero sum of the total changes, i.e., $\sum_{i=1}^{9} \Delta p_{i}=0$, where $\Delta p_{i}(i=1, \cdots, 9)$ is the change applied individually to each controlled parameter. Therefore, any eight of the nine control parameters are independent, and the ninth parameter is linearly dependent upon the other eight parameters.

\subsection{Base Scenario and Modeling Assumptions}

The major assumptions that form the basis of the system assessment study are load and generation. As required in the North American Electric Reliability Corporation (NERC) Reliability Standards, the 
transmission system is planned for expected peak load conditions. The case was prepared by BPA (2007 heavy summer case). The modeling assumptions are listed as follows:

- Loads are forecasted for peak summer, peak winter, and abnormal peak winter conditions. Normal summer and winter peak loads were based on a probability of 50 percent not to exceed the target load peak, while extreme peak loads for abnormal winter conditions were based on a probability of 95 percent not to exceed the target load peak. The summer peak load in WECC 2007 heavy summer case is 154, 208 MW.

- High water year (2007). The shortage of generation in California will be made up with imports from Northwest and British Columbia regions with high hydro capability.

\subsection{Contingencies and Stressing Procedures}

The main components of the approach include contingency screening, computation of maximum loading point, and building the security nomogram.

The analysis of all contingencies is too time-consuming and may be not necessary in practice. Our numerical experiment uses a short contingency list specially composed to analyze security conditions of the BPA system. The contingency list consisting of 366 predefined contingencies was used in the simulation.

Some of the descriptor parameters correspond to the transfer capability between selected WECC subsystems. Total transfer capability (TTC) is the maximum power that can be transmitted between subsystems without violating physical limits of the transmission grid.

Our simulation starts from the base case. Once the generation and load stress patterns (given by participation factors) are assumed, the transfer capability is calculated by changing these controlled parameters to cause additional power exchange between the subsystems (that is, to stress the descriptor parameters). The power generation is increased at the export area and decreased at the import area. The procedure is stopped when the security conditions are violated. Changes in the selected descriptor parameter (power transfer) can be also caused by load variations in subsystems following the specified stress pattern.

All base cases used in this study were developed starting from the WECC approved 2007 heavy summer operating base case.

\subsubsection{Reliability Criteria}

All study results were evaluated using the WECC reliability criteria [37], the WECC voltage stability criteria, and member reliability criteria as appropriate. Accordingly, a Category B disturbance in one system shall not cause a transient voltage dip in another system that is greater than $20 \%$ for more than 20 cycles at load buses, or exceed $25 \%$ at load buses or $30 \%$ at non-load buses at any time other than during the fault. This disturbance also can not cause the frequency to drop below $59.6 \mathrm{~Hz}$ for six cycles 
or more at a load bus. This disturbance standard, which all WECC member system must comply with, is listed in Table 3-1 and illustrated in Figure 3.2.

Transient stability simulations (TSS) are run out to 20 seconds. TSS $100 \mathrm{MW}$ stability test is applied to each nomogram point (positive damping at power limit $+100 \mathrm{MW}$ ). Transient stability simulations are performed using the dynamics data file issued with the WECC approved 2007 heavy summer operating base case. An existing remedial action scheme (RAS) called Fast AC Reactive Insertion (FACRI) at BPA's Malin 500-kV substation is modeled as available for all contingencies; insertion of the series capacitors at Fort Rock and the shunt capacitors at Malin occurs if the Malin 500-kV voltage drops to the design levels. PNW RAS, including generation tripping, are modeled as designed for the specific contingency. The shortened contingency list (366 contingencies) includes those contingencies that are most probably critical.

Based on WECC reliability requirement, reactive margin is evaluated through use of the TSS power margin test. The test was applied to all nomogram points (power limit $+5 \%$ for $\mathrm{N}-1$ and power limit + $2.5 \%$ for $\mathrm{N}-2$ ). The system must have positive margin at the power test points. (All contingencies will be screened at the $5 \%$ point; the $2.5 \%$ test will be run only if there are $\mathrm{N}-2$ contingencies that do not solve at the 5\% test) Pacific Northwest (PNW) RAS for N-1 and N-2 contingencies are modeled as designed for the specific contingency. No system element will be loaded more than $100 \%$ of the emergency thermal rating (non-BPA) following a contingency. For BPA equipment, no element will be allowed to reach its maximum operating temperature within 20 minutes. RAS load dropping in the Phoenix area for loss of units 1 and 2 or units 2 and 3 at Palo Verde is modeled.

Table 3-1. WECC Disturbance-Performance Table of Allowable Effects on Other Systems [37]

\begin{tabular}{|c|c|c|c|c|}
\hline $\begin{array}{l}\text { NERC and } \\
\text { WECC } \\
\text { Categories }\end{array}$ & $\begin{array}{c}\text { Outage Frequency } \\
\text { Associated with the } \\
\text { Performance Category } \\
\text { (outage/year) }\end{array}$ & $\begin{array}{l}\text { Transient Voltage } \\
\text { Dip Standard }\end{array}$ & $\begin{array}{l}\text { Minimum } \\
\text { Transient } \\
\text { Frequency } \\
\text { Standard }\end{array}$ & $\begin{array}{c}\text { Post Transient } \\
\text { Voltage Deviation } \\
\text { Standard }\end{array}$ \\
\hline A & Not Applicable & $\begin{array}{l}\text { Nothing in addition to } \\
\text { NERC }\end{array}$ & & \\
\hline B & $\geq 0.33$ & $\begin{array}{l}\text { Not to exceed } 25 \% \text { at } \\
\text { load buses or } 30 \% \text { at } \\
\text { non-load buses. Not } \\
\text { to exceed } 20 \% \text { for } \\
\text { more than } 20 \text { cycles } \\
\text { at load buses. }\end{array}$ & $\begin{array}{l}\text { Not below } 59.6 \mathrm{~Hz} \\
\text { for six cycles or } \\
\text { more at a load bus. }\end{array}$ & $\begin{array}{l}\text { Not to exceed } 5 \% \\
\text { at any bus. }\end{array}$ \\
\hline $\mathrm{C}$ & $0.033-0.33$ & $\begin{array}{l}\text { Not to exceed } 30 \% \text { at } \\
\text { any bus. Not to } \\
\text { exceed } 20 \% \text { for more } \\
\text { than } 40 \text { cycles at } \\
\text { load buses. }\end{array}$ & $\begin{array}{l}\text { Not below } 59.0 \mathrm{~Hz} \\
\text { for } 6 \text { cycles or } \\
\text { more at a load bus. }\end{array}$ & $\begin{array}{l}\text { Not to exceed } 10 \% \\
\text { at any bus. }\end{array}$ \\
\hline $\mathrm{D}$ & $<0.033$ & $\begin{array}{l}\text { Nothing in addition to } \\
\text { NERC }\end{array}$ & & \\
\hline
\end{tabular}




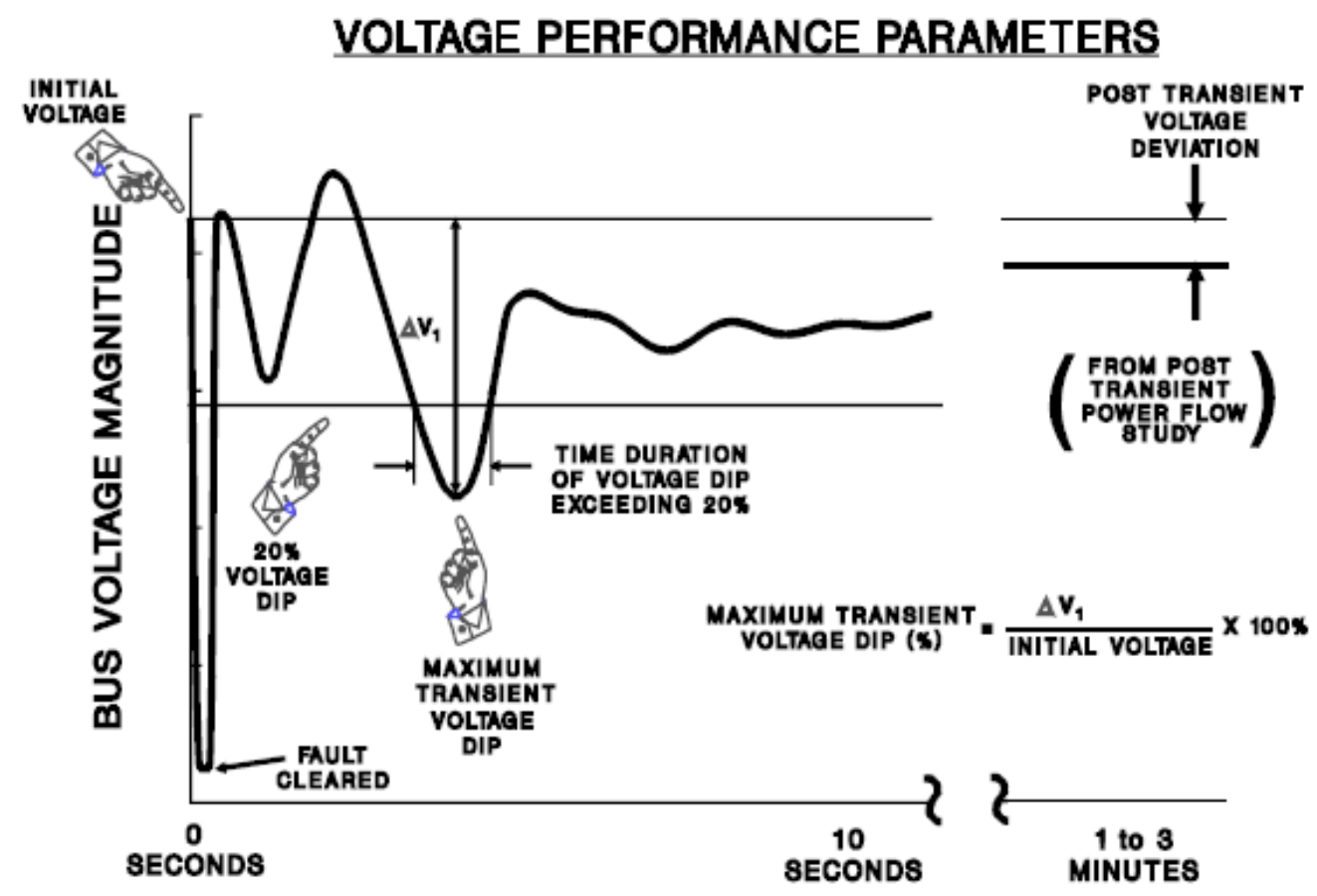

TIME

Figure 3.2. Voltage Performance Parameters [37]

\subsubsection{Simulation Procedures to Find Security Boundary Points}

This section describes the procedures to find the security boundary points, based on which the security hyperplanes are developed.

\subsubsection{Simulation Procedures}

In accordance with the NERC/WECC Planning Standards, the following reliability performance standards/criteria are applied

a. System transiently and dynamically stable

b. System with adequate reactive margin to protect against voltage collapse

c. All transmission facilities within their thermal ratings.

The procedure to find a boundary point of the security region consists of several steps, which includes

1) Select the base case.

2) Build the database of contingency list.

3) Perform contingency analysis while increasing power along the stress direction until any violation of thermal, voltage, transient stability or other limits occur.

4) Record the boundary point found. 
The basic simulation procedures to perform power flow, transient stability simulation and posttransient calculation are illustrated in Figure 3.3.

A. The simulation starts from 2007 heavy summer case;

B. Develop a series of system base conditions (case $\alpha$ ) with increasing transfer path or load/generation;

C. Create the $105 \%$ and $102.5 \%$ transfer flow scenario for each base case (case $\alpha .1$ and $\alpha .2$ );

D. For each scenario ( $\alpha .1$ and $\alpha .2)$, select contingencies $(\mathrm{N}-1$ and $\mathrm{N}-2)$, run post-transient power flow, and evaluate the disturbance performance against WECC Reliability criteria

1. The system must have at least $5 \%(2.5 \%)$ positive margin when $\mathrm{N}-1(\mathrm{~N}-2)$ contingency is applied

2. No element will be allowed to be loaded more than $100 \%$ of the emergency thermal rating after disturbance

3. Post-transient voltage deviation cannot exceed $5 \%$ at any bus

The post-transient power flow is calculated by Power World ${ }^{\mathrm{TM}}$, which provides good models for the equipment operating limits (generator capability limits, transformer tap changes, circuit ratings and bus voltage criteria) and discrete controls (transformer tap steps and switched shunts).

E. Starting from the base case (case $\alpha$ ), increase interface power flow by $100 \mathrm{MW}$ (case $\alpha .3$ ), run transient stability simulation with same contingencies as in step D and check the transient stability requirements

1. Disturbance shall not cause a transient voltage dip in another system that is greater than $20 \%$ for more than 20 cycles at load buses, or exceed $25 \%$ at load buses or $30 \%$ at non-load buses at any time other than during the fault.

2. Disturbance cannot cause the frequency to be below $59.6 \mathrm{~Hz}$ for six cycles or more at a load bus.

Transient stability simulations are performed by General Electric Positive Sequence Load Flow (PLSF) program. Programs for modeling of power system equipment were written in EPCL control language.

F. If all requirements are satisfied for case $\alpha$, go back to the step B, stress the system further, and repeat this procedure until one violation is reached. Otherwise, the previous satisfactory case is identified as one nomogram point. 
A. Start from 2007 heavy summer case

$\downarrow$

B. Develop a series of system base conditions (case $\alpha$ ) with increasing transfer path or load/generation

C. Create the $105 \%$ and $102.5 \%$ transfer flow scenario for each base case (case $\alpha .1$ and $\alpha .2$ )

D. For each scenario ( $\alpha .1$ and $\alpha .2)$, select contingencies $(\mathrm{N}-1$ and $\mathrm{N}-2$ ), run post-transient power flow, and evaluate the disturbance performance against WECC reliability criteria

1) The system must have at least $5 \%(2.5 \%)$ positive margin when $\mathrm{N}-1(\mathrm{~N}-2)$ contingency is applied

2) No element will be allowed to be loaded more than $100 \%$ of the emergency thermal rating after disturbance

3) Post-transient voltage deviation can not exceed 5\% at any bus

E. Starting from the base case (case $\alpha$ ), increase interface power flow by $100 \mathrm{MW}$ (case $\alpha .3$ ), run transient stability simulation and check the transient stability requirements

1) Disturbance shall not cause a transient voltage dip in another system that is greater than $20 \%$ for more than 20 cycles at load buses, or exceed $25 \%$ at load buses or $30 \%$ at non-load buses at any time other than during the fault

2) Disturbance can not cause the frequency to be below $59.6 \mathrm{~Hz}$ for six cycles or more at a load bus.

F. If all requirements are satisfied for case $\alpha$, go back to the step B, stress the system further, and repeat this procedure until one violation is reached. Otherwise, the previous satisfactory case is identified as one nomogram point.

Figure 3.3. Simulation Procedures 


\subsubsection{Refined Procedure to Find Security Boundary Points}

As we described above, each contingency must be checked for each increment in the system parameters along the stress direction. This is a very computation demanding. The efficiency of simulation can be improved if the contingency list is categorized as critical and non-critical ones based on engineering judgment. The critical contingencies, which cause the bottleneck in the transmission system, usually impose more stresses to power system than the non-critical ones. This design can lead to lots of savings in the man-power or computation resources. The idea is briefly explained as follows (see Figure 3.4).

1. Divide contingency list into critical and non-critical ones.

2. Stress the system along the specified direction. Conduct contingency analysis at each incremental step using the critical contingencies. Stop when the violations of reliability criteria occur.

3. Record the limiting point, and check whether the system is secure or not with non-critical contingencies. If yes, this recorded point represents a boundary point on the security region boundary. If no, move the violated contingency from non-critical set to critical one and repeat step from (1) to (3) until all contingencies have been checked satisfactorily before reaching the limiting point.

Suppose the number of the contingency list is $N$, and it takes $M$ steps to stress the system from the base point to one boundary point. To build a hyperplane in $\mathrm{K}$ dimensions $(\mathrm{K}=8)$, at least $\mathrm{K}$ boundary points are needed. $\mathrm{K} \times \mathrm{N} \times \mathrm{M}$ simulation runs are needed in total to obtain one hyperplane. The situation becomes worse if the new approach is targeted at $\mathrm{K}$ different paths $(\mathrm{K}=8)$. Because the transfer capability along each path is usually constrained by the different stress pattern (or outage), different contingency lists need to be applied when the different paths are considered. Suppose the number of the contingency list for

eight different paths are $\mathrm{N}_{1}, \mathrm{~N}_{2} \cdots \mathrm{N}_{\mathrm{K}}$. Therefore, $\mathrm{K} \times \sum_{i=1}^{k} N_{i} \times \mathrm{M}$ simulation run is needed. The simulation effort is formidable if not simplified.

In view of these considerations, the new simulation steps are proposed.

1) Select the critical outages for the targeted path

The outages selected to be applied are one of the most important variables in this stress test. As discussed, the most critical outage associated with different path is different because the stress direction will be different. Either based on the severity ranking or engineering judgment, a list of the credible and critical outages can be selected for each target path. By doing so, the number of outages has been significantly reduced from $N_{i}$ to $R_{i}\left(N_{i} \gg R_{i}\right)$.

2) Apply contingency analysis based on the critical outage list $R_{i}$ to find the boundary point. Suppose it takes $M$ steps to stress the system from the base point to one boundary point. The simulation step required is $\mathrm{M} \times R_{i}$ 
3) Check whether the system is secure or not at the boundary point for non-critical contingency $\sum_{i=1}^{k} N_{i}-R_{i}$. If the system can perform satisfactorily under all non-critical contingency, move to the next step (in this case, the simulation step is totally $\mathrm{M} \times R_{i}+\sum_{i=1}^{k} N_{i}-R_{i}$ ); otherwise add the violated contingency into the critical outrage list, and restart from step 1).

4) Obtain $\mathrm{K}$ boundary points $(\mathrm{K}>8)$ and build the hyperplane from the boundary points.

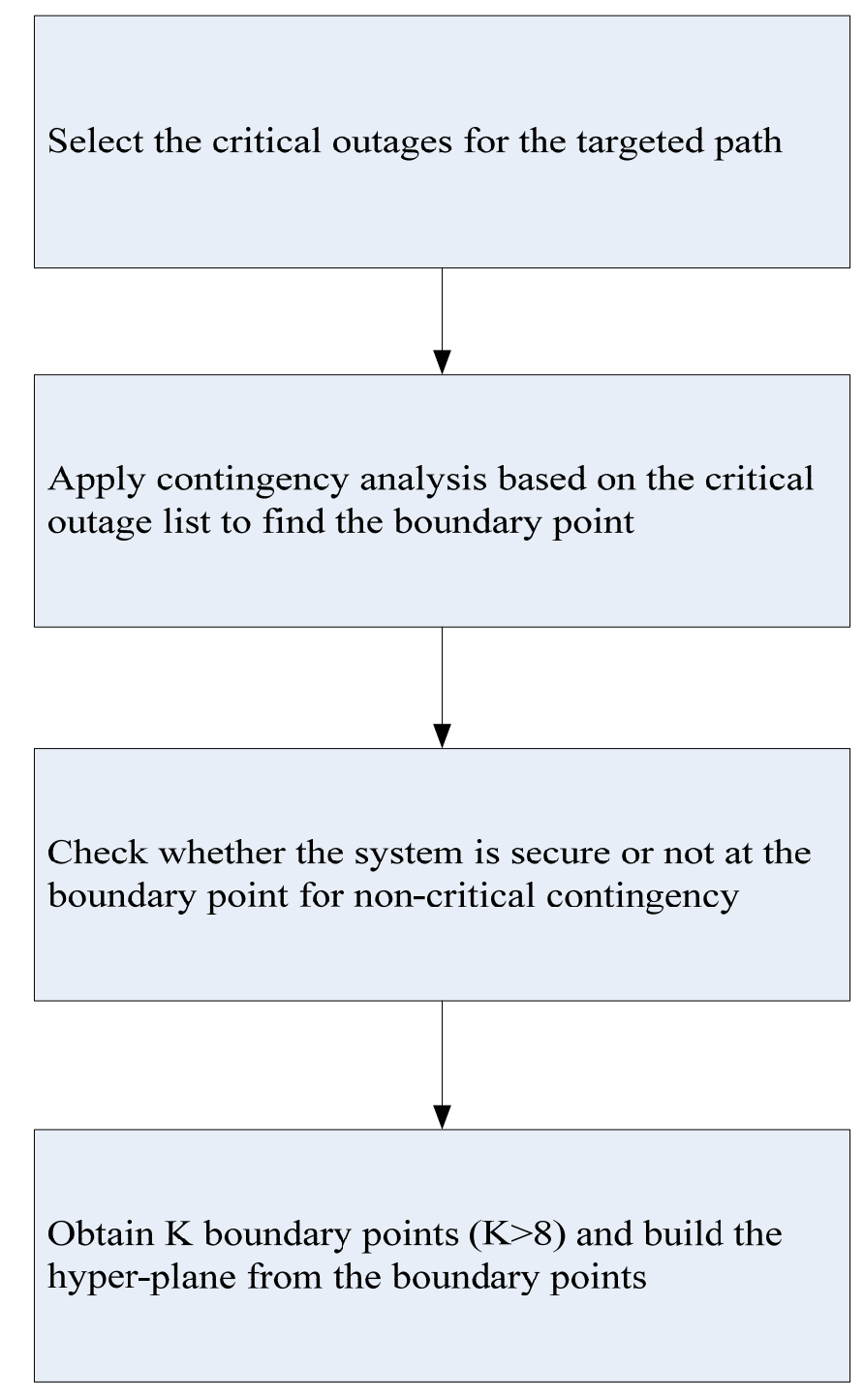

Figure 3.4. Flowchart of Refined Simulation Steps to Find a Boundary Point 


\subsubsection{Simulation Procedures to Build Security Region in a Multi-dimension Parameter Space}

After the security boundary points have been found, the hyperplanes can be built in an iterative way, as described in the Section 2.3.

\subsubsection{Automatic Simulation Procedures in PowerWorld SimAuto}

The simulation procedure to explore the whole security region was implemented in PowerWorld SimAuto add-on module. Essentially, the code is called from Matlab with an interface with SimAuto (see Figure 3.6 Automatic Simulation Procedures in SimAuto for flow chart of this code). It has three components: to find the boundary point, to build the hyperplane and to refine the hyperplanes. After this new tool has been developed, the approach proposed can be generally applied to any system and any operation condition with a minimum effort of modification. It can be running automatically without requiring the intervention from users. Together with the categorization of critical and non-critical contingencies, the simulation speed has been significantly increased. It is possible that any significant change to the system operation conditions predicated several hours ahead can be taken into consideration, and the hyperplanes can be rebuilt in a timely manner. This further opens up new opportunity of using wide-area security nomograms for on-line security assessment. This possibility will be discussed more in the following section. 


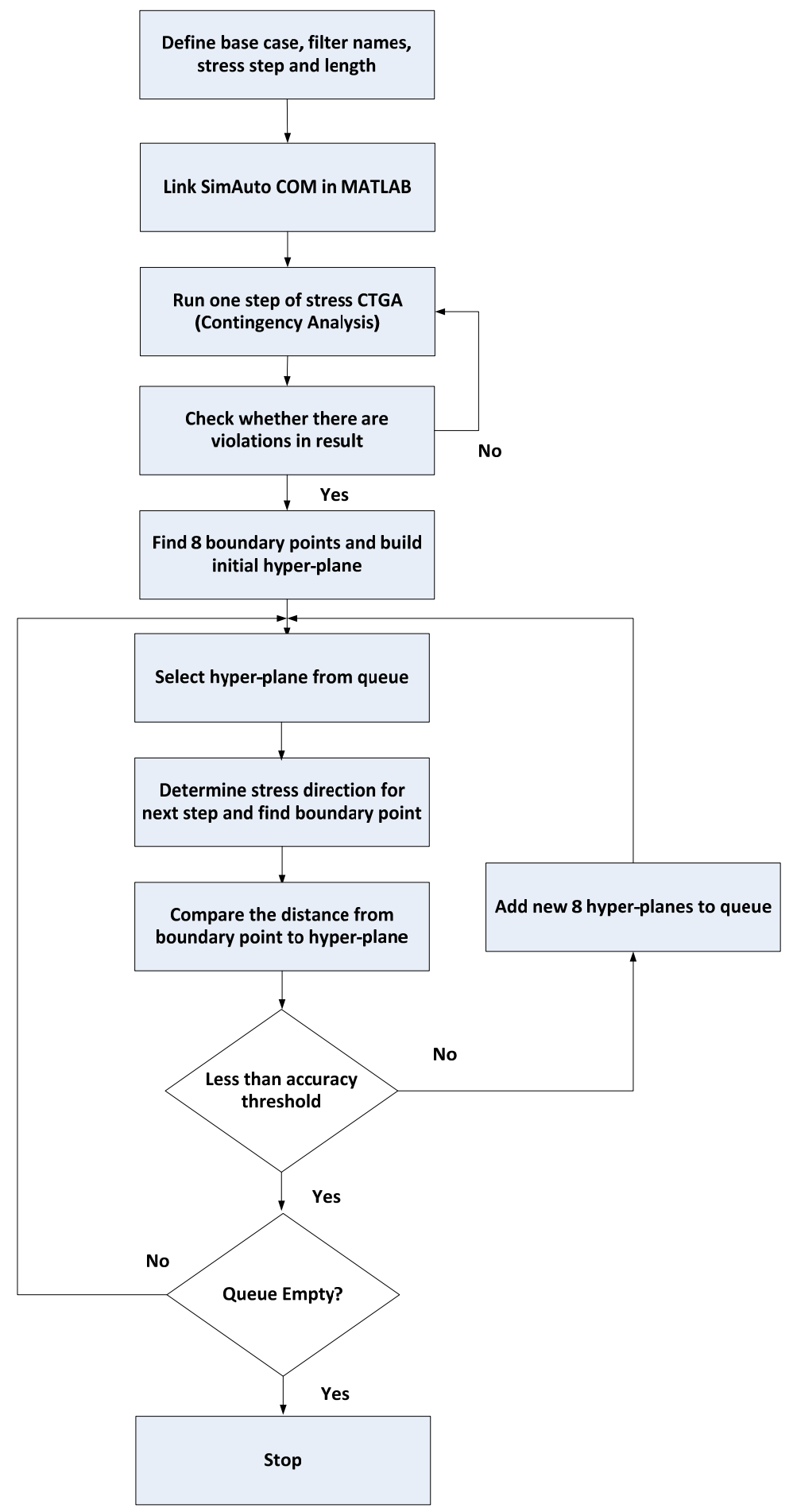

Figure 3.5. Automatic Simulation Procedures in PowerWorld SimAuto 


\subsection{Numerical Results}

This section provides results of our simulation study and the corresponding nomograms.

\subsubsection{All HyperPlanes}

The coefficients of 92 hyperplanes are shown in the Appendix B.

\subsubsection{Validation of Tangent Hyperplanes}

The selected tangent hyperplanes were validated in two ways

1) By measuring the distance from the tangent hyperplanes to the accurate security boundary

2) By comparing the tangent hyperplane with the existing nomograms used by BPA.

\subsubsection{Accuracy of Hyperplanes}

To measure the difference between a security boundary point $\bar{D}$ and its approximation using a hyperplane, the absolute error $\varepsilon_{d}$ of approximation is defined as

$$
\varepsilon_{d}=\frac{\left|N_{d}^{t} \cdot \bar{D}-L_{d}\right|}{L_{d}} 100 \%
$$

where $\bar{D}$ is the boundary point, e.g., calculated using a gradual system stress in a given direction, and $N_{d}^{t}$ and $L_{d}$ are coefficients of the hyperplane.

Table 3-2. and Table 3-3 provide parameters for three hyperplanes selected from Table B. $1^{11}$. The maximum absolute error of the hyperplane is $\varepsilon_{d}=1.71 \%$. It indicates that the hyperplanes built is very accurate in approximating the nonlinear security boundary.

Table 3-2. Normal Vectors of Hyperplanes in the Control Parameter Space

\begin{tabular}{cccccccccc}
\hline & $\begin{array}{c}\text { Area1 } \\
\text { Generation }\end{array}$ & $\begin{array}{c}\text { Area2 } \\
\text { Generation }\end{array}$ & $\begin{array}{c}\text { Area3 } \\
\text { Generation }\end{array}$ & $\begin{array}{c}\text { Area4 } \\
\text { Generation }\end{array}$ & $\begin{array}{c}\text { Area5 } \\
\text { Generation }\end{array}$ & $\begin{array}{c}\text { Area6 } \\
\text { Load }\end{array}$ & $\begin{array}{c}\text { Area7 } \\
\text { Load }\end{array}$ & $\begin{array}{c}\text { Area8 } \\
\text { Load }\end{array}$ & $L_{c}$ \\
\hline 1 & 0.42 & 0.43 & 0.12 & 0.28 & 0.43 & -0.42 & -0.29 & -0.29 & 2061 \\
2 & 0.44 & 0.46 & 0.10 & 0.32 & 0.48 & -0.45 & -0.17 & -0.15 & 1673 \\
3 & 0.34 & 0.36 & 0.10 & 0.44 & 0.39 & -0.42 & -0.32 & -0.34 & 1736 \\
\hline
\end{tabular}

\footnotetext{
${ }^{11}$ Hyperplanes No. 86, 91 and 92 in Table B.1
} 
Table 3-3. Normal Vectors of Hyperplanes in the Descriptor Parameter Space

\begin{tabular}{cccccccccc}
\hline & $d_{1}$ & $d_{2}$ & $d_{3}$ & $d_{4}$ & $d_{5}$ & $d_{6}$ & $d_{7}$ & $d_{8}$ & $L_{d}$ \\
\hline 1 & -0.07 & 0.05 & -0.48 & 0.69 & 0.31 & 0.10 & -0.39 & -0.13 & 5047 \\
2 & 0.0 & 0.0 & -0.31 & 0.0 & 0.95 & 0.0 & 0.0 & 0.0 & 7306 \\
3 & 0.0 & 0.0 & -0.60 & 0.80 & 0.0 & 0.0 & 0.0 & 0.0 & 3471 \\
\hline
\end{tabular}

\subsubsection{Comparison of Hyperplanes with Existing Nomograms}

The mathematical description of the determined hyperplanes is as follows:

Hyperplane $1 H_{c, 1}$ (in the control parameter space): ${ }^{12}$

$$
0.42 c_{1}+0.43 c_{2}+0.12 c_{3}+0.28 c_{4}+0.43 c_{5}-0.42 c_{6}-0.29 c_{7}-0.29 c_{8}=2061
$$

The same hyperplane $1 H_{d, 1}$ (in the descriptor parameter space): ${ }^{13}$

$$
-0.07 d_{1}+0.05 d_{2}-0.48 d_{3}+0.69 d_{4}+0.31 d_{5}+0.1 d_{6}-0.39 d_{7}-0.13 d_{8}=5047
$$

BPA is responsible for determining transfer capability north of the California-Oregon border (COB). The California ISO studies COI capability south of COB. Capability from the border south is defined by a nomogram relationship that includes area generation and area load. The most restrictive conditions for the transfers across $d_{4}, d_{5}$, and another Intertie occur when flow on $d_{3}$ is in a particular direction. The 2008 summer nomogram provided by BPA is shown in Figure 3.8 and its three-dimensional view is shown in Figure 3.9. It is worthwhile to point out that BPA study concludes that the 2007 summer nomogram is the same as the 2008 summer nomogram.

To compare with that result, the hyperplanes are projected on the space that consists of $d_{4}, d_{5}$ and $d_{3}$. This is achieved by assigning other five parameters the typical values of one boundary point of the nomogram. The hyperplanes in the three-dimension space become

$$
\begin{gathered}
-0.48 d_{3}+0.69 d_{4}+0.31 d_{5}=5231^{14} \\
-0.31 d_{3}+0.95 d_{5}=7306 \\
-0.60 d_{3}+0.80 d_{4}=3471
\end{gathered}
$$

Define $d_{4}^{*}=d_{4}+150 \mathrm{MW} .{ }^{15}$ The hyperplanes expressed in the variables of $d_{4}^{*}, d_{5}$, and $d_{3}$ are

\footnotetext{
${ }^{12} c_{1}$ : Area1 generation; $c_{2}$ : Area2 generation; $c_{3}$ : Area3 generation; $c_{4}$ : Area4 generation; $c_{5}$ : Area5 generation; $c_{6}$ : Area6 load; $c_{7}$ : Area7 load; $c_{8}:$ Area8 load.

${ }^{13} d_{1}$ : Flow1; $d_{2}$ : Flow2; $d_{3}$ : Flow3; $d_{4}$ : Flow4 $d_{5}$ : Flow5; $d_{6}$ : Flow6; $d_{7}$ : Flow7; $d_{8}$ : Flow8

${ }^{14} d_{1}=-2619 \mathrm{MW} ; d_{2}=1143 \mathrm{MW} ; d_{6}=4221 \mathrm{MW} ; d_{8}=2953 \mathrm{MW} ; d_{7}=1239 \mathrm{MW}$.

${ }^{15}$ A North-South Path power flow equals to $150 \mathrm{MW}$ for all simulation cases.
} 


$$
\begin{gathered}
-0.48 d_{3}+0.69 d_{4}^{*}+0.31 d_{5}=5334^{16} \\
-0.31 d_{3}+0.95 d_{5}=7306 \\
-0.60 d_{3}+0.80 d_{4}^{*}=3591
\end{gathered}
$$

These hyperplanes described by (Equation (3.5)) are shown in Figure 3.6 and Figure 3.7.

The comparison shows that

1. The most influential factors in determining the security region are $d_{4}^{*}, d_{5}$, and $d_{3}$.

2. Our simulation results show that the maximum capability of the $d_{5}$ cut plane is $7718 \mathrm{MW}$, which is less than BPA study, i.e., $7800 \mathrm{MW}$. The source of error resulting in the more conservative estimation will be explored in Section 3.6.

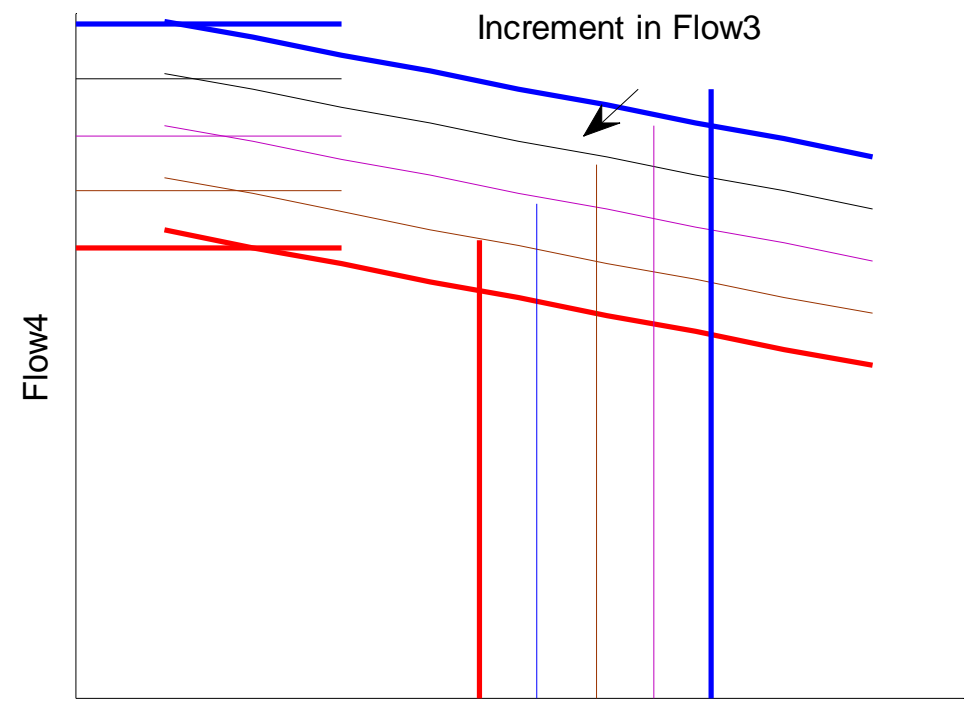

Flow5

Figure 3.6. 2007 Heavy Summer Nomogram Predicted from Hyperplane

${ }^{16} d_{4}^{*}=d_{4}+$ A North-South path. 


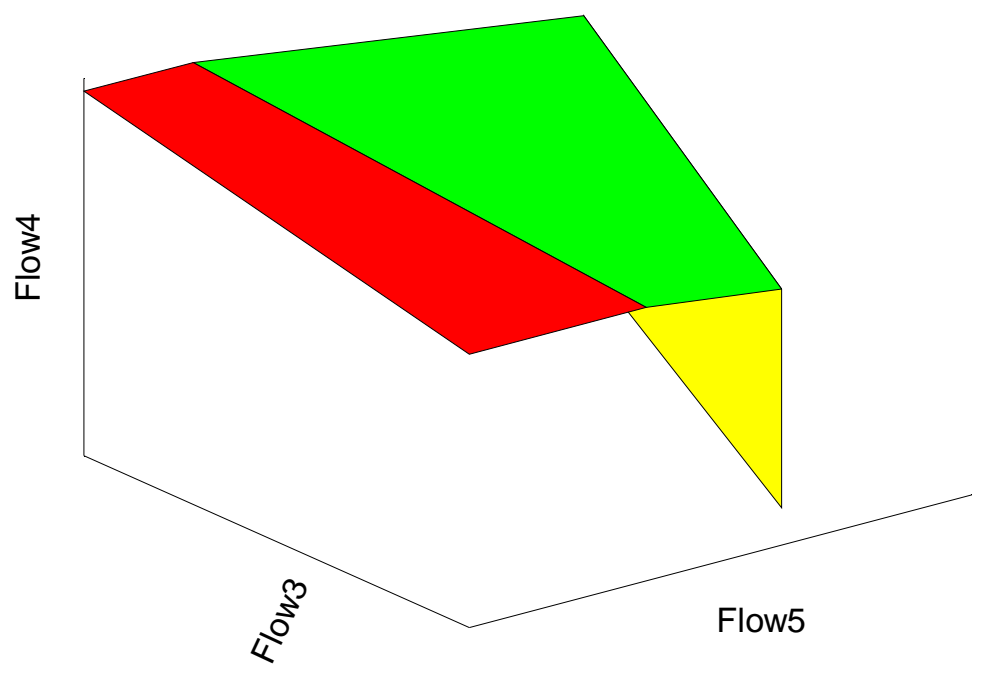

Figure 3.7. Three-dimensional View of 2007 Summer Nomogram Projected from Hyperplane ${ }^{17}$

${ }^{17}$ Provided by H. Johannsson 


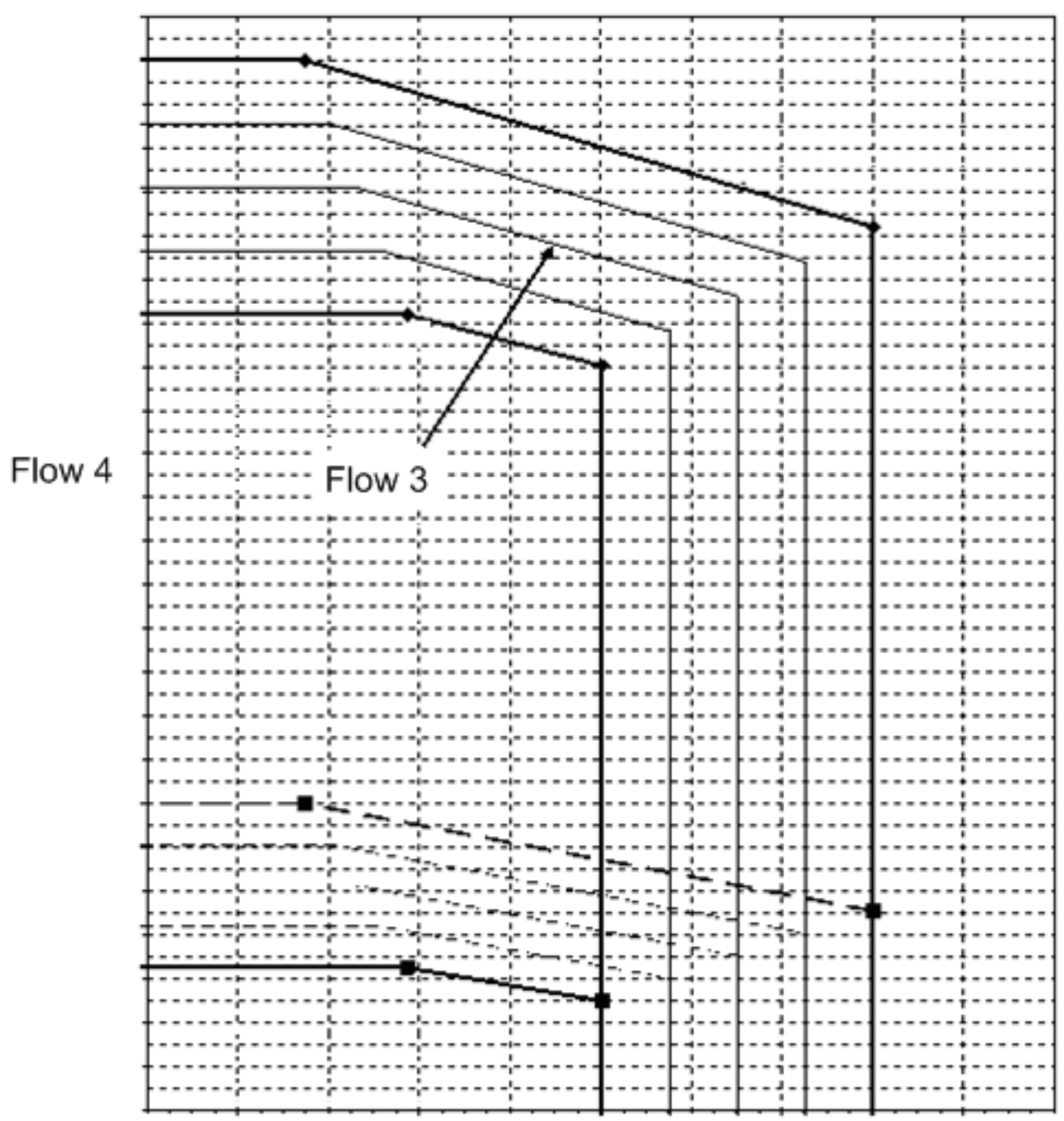

Flow 5

Figure 3.8. 2008 Summer Nomogram Provided by BPA 


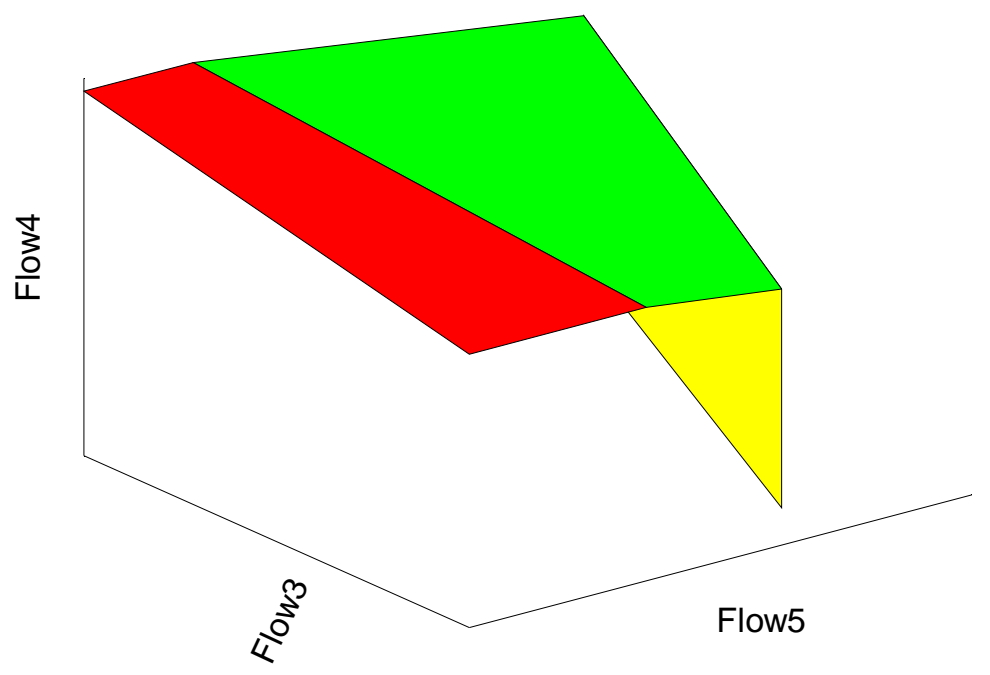

Figure 3.9. Three-dimensional View of 2008 Summer Nomogram Provided by BPA

\subsection{Express-analysis of the Security Margin}

The distance $\xi_{d}$ from the current operation point $D$ to the nearest constraint facing $D$ determines the security margin.

$$
\xi_{d}=\frac{\eta_{d, 1} d_{1}+\eta_{d, 2} d_{2}+\cdots+\eta_{d, n_{d}} d_{n_{d}}-L_{d}}{\left\|N_{d}\right\|}
$$

where the current operating point is $D=\left[\begin{array}{llll}d_{1} & d_{2} & \cdots & d_{n_{d}}\end{array}\right]$, and the nearest constraint facing $D, H_{d}$, is $\eta_{d, 1} d_{1}+\eta_{d, 1} d_{2}+\cdots+\eta_{d, n_{d}} d_{n_{d}} \leq L_{d}$

Assuming that the projection of $D$ on the hyperplane $H_{d}$ is $P_{B}$, vector $\overrightarrow{P_{B} D}$ is perpendicular to the hyperplane $\Psi$, as shown in Figure 3.10. Point $P_{B}$ can be found by applying the following formula:

$$
P_{B}=D+\xi_{d} \cdot \frac{N_{d}}{\left\|N_{d}\right\|}
$$




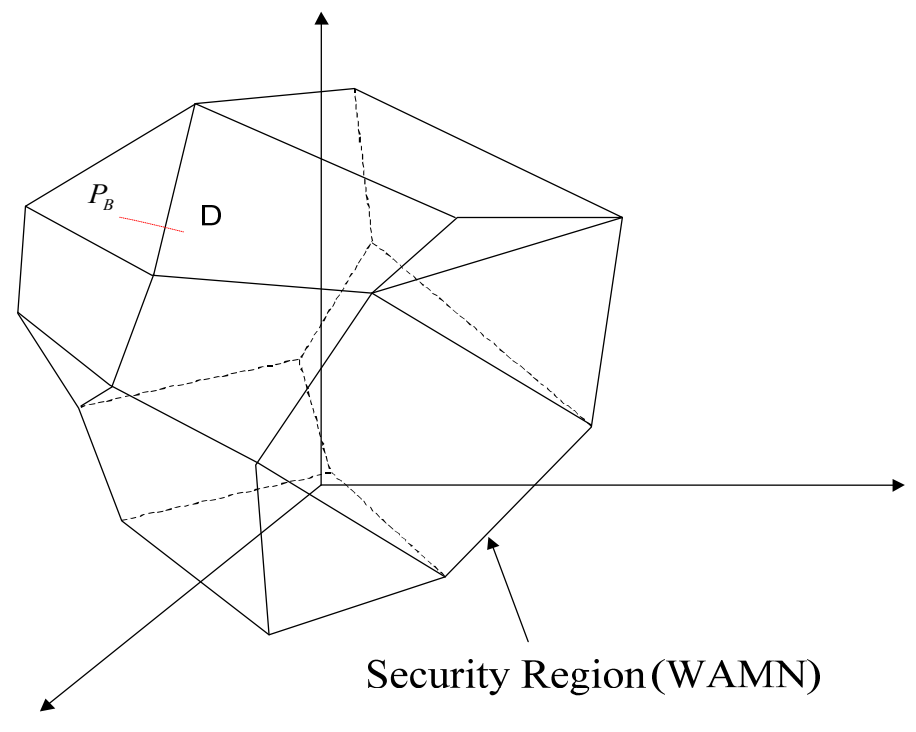

Figure 3.10. Calculation of Security Margin

The first base case point is chosen as $D_{0}$ in the descriptor parameter space. The security margin to $H_{d, 1}$ is calculated as $\xi_{d}=268 \mathrm{MW}$. To validate this result, a simulation is carried out with the new stress direction, which is chosen such that it causes the descriptor parameters to move in the same direction as vector $N_{d}$. The maximum power transfer condition $P_{B 0}^{\prime}$, obtained as a simulation result, yields a security margin of $292 \mathrm{MW}$, as listed in Table 3-4.

Table 3-4 provides a summary of results. It shows that the security margin predicted from the hyperplane and results from simulations are close.

Table 3-4. Calculation of Security Margin for Operation Point $D_{0}$

\begin{tabular}{cccccccccc}
\hline & $d_{1}$ & $d_{2}$ & $d_{3}$ & $d_{4}$ & $d_{5}$ & $d_{6}$ & $d_{7}$ & $d_{8}$ & $\begin{array}{c}\text { Security } \\
\text { Margin }\end{array}$ \\
\hline$D_{0}$ & -2620 & 1154 & 93 & 4047 & 7380 & 4058 & 1212 & 2905 & \\
$P_{B 0}$ & -2638 & 1166 & -35 & 4233 & 7463 & 4086 & 1106 & 2869 & $268 \mathrm{MW}$ \\
$P_{B 0}^{\prime}$ & -2620 & 1125 & -35 & 4259 & 7434 & 4173 & 1153 & 2967 & $292 \mathrm{MW}$ \\
\hline
\end{tabular}




\subsection{Sensitivities Analysis}

The distance $\xi_{d}$ from the current operation point $D$ to the nearest constraint facing $D$ (i.e., the security margin), is denoted by

$$
\xi_{d}=\frac{\left|\eta_{d, 1} d_{1}+\eta_{d, 2} d_{2}+\cdots+\eta_{d, n_{d}} d_{n_{d}}-L_{d}\right|}{\left\|N_{d}\right\|}
$$

The sensitivities of the parameters to the security margin is given by

$$
\frac{\partial \xi_{d}}{\partial d_{i}}=\frac{\eta_{d, i}}{\left\|N_{d}\right\|} \quad\left(i=1,2, \cdots, n_{d}\right)
$$

The sensitivities of the change of parameters with respect to the security margin for hyperplanes, $H_{c, 1}$ and $H_{d, 1}$, are shown in Figure 3.11 and Figure 3.12, respectively. These normalized coefficients of the set of hyperplane equations are sensitivities that can be interpreted in several ways. In the descriptor parameter space, the sensitivities can suggest the most limiting elements of grid. In the control parameter space, the sensitivities reveal the locations in the network where the most sensitive controls are needed.

In Figure 3.11, the sensitivities of the control parameters for hyperplane $H_{c, 1}$ are plotted. It shows that the Area5 generation has the most significant impact on the security margin, and this is the most effective control to increase the security margin. The effects of generations and loads are different. It is because that the increment in the generation or the reduction in the load will result in more power transferred from the North to the South, which stresses the bottleneck $\left(d_{4}\right)$ further.

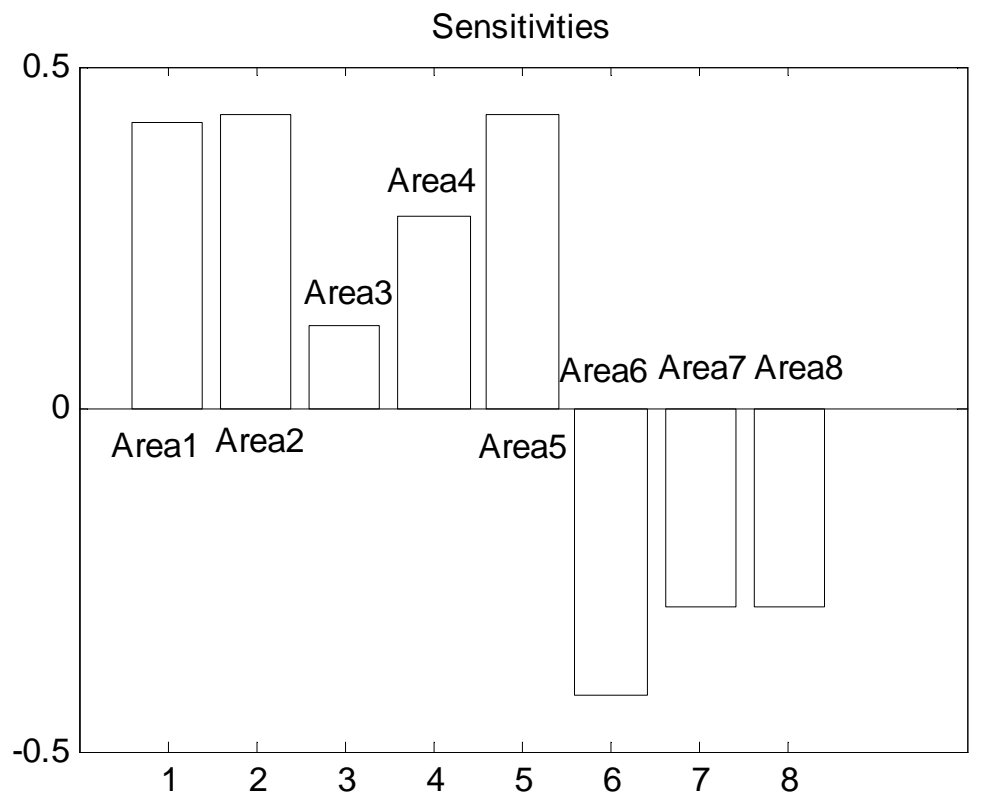

Figure 3.11. Sensitivities of Change of Generation/Load with Respect to Security Margin 
In Figure 3.12, the sensitivities of the descriptor parameters for hyperplane $H_{d, l}$ are plotted. The highest value is the sensitivities of $d_{4}$ to the security margin. This is consistent with the fact that hyperplane $H_{d, 2}$ is built when the power from North to South reaches the maximum operating transfer capability.

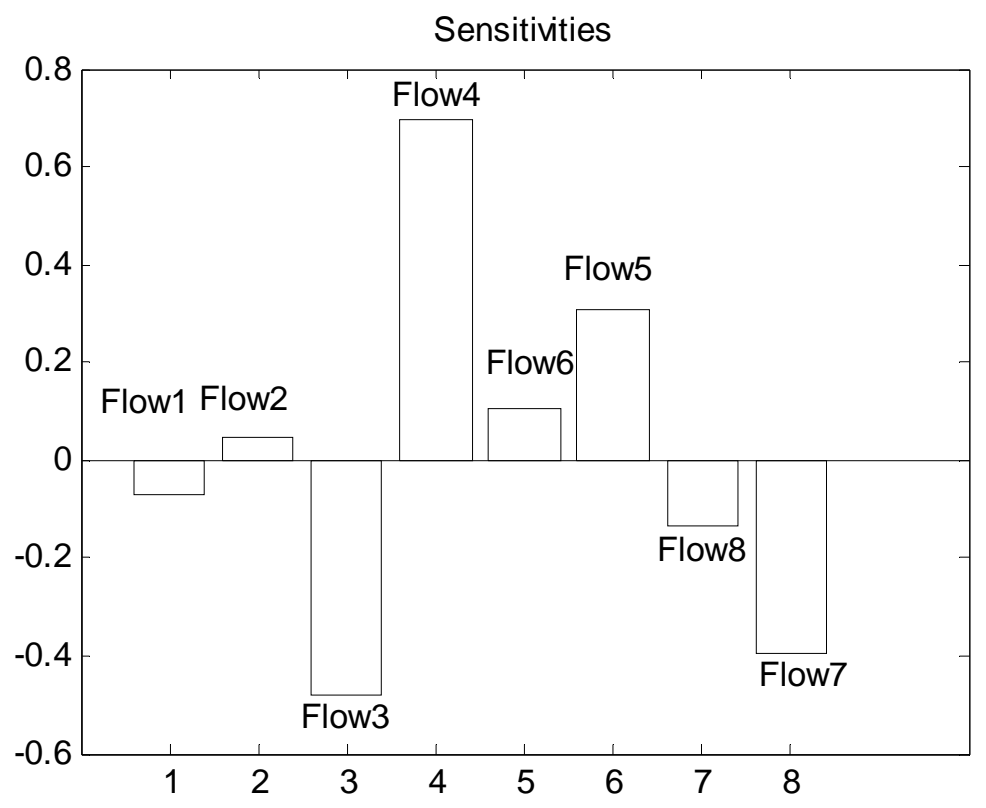

Figure 3.12. Sensitivities of Hyperplane in Descriptor Parameter Space

The sensitivity analysis indicates that the $d_{7}$ has a considerable effect in determining the security region of BPA system. This can be illustrated by Figure 3.13. If the Area6 load, Area7 load, Area8 load or their combination changes, different boundary condition can be obtained from the simulation. ${ }^{18}$ Thus, it can be assumed that if the five descriptor parameters $\left(d_{1}, d_{2}, d_{6}, d_{7}\right.$, and $\left.d_{8}\right)$ are fixed and the same as new boundary condition, which is different from the condition in Figure 3.6, the new hyperplane in the space of $d_{3}, d_{4}$, and $d_{5}$ is

$$
-0.48 d_{3}+0.69 d_{4}+0.31 d_{5}=5263
$$

This hyperplane is plotted as the dotted lines in Figure 3.13, and the solids lines are the same hyperplane as in Figure 3.6.

${ }^{18} L_{d}$ is calculated based the condition $D_{1}=[-26201144764341421975962971$ 1248]. 


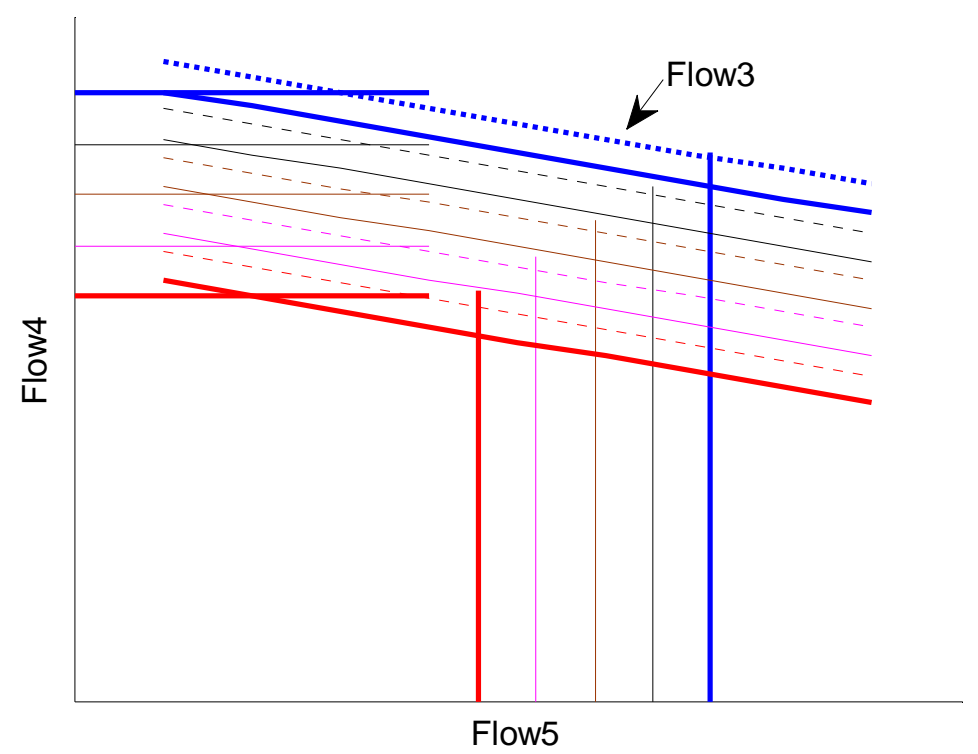

Figure 3.13. Comparison between the Hyperplanes with Different Power Flow in the Interface of the Flow7

The shift from solid lines to dotted lines in Figure 3.13 reflects the impact of the other descriptor parameters, which are not included in the figure, on $d_{4}$ and $d_{5}$ limits. It should also be the difference in values of these parameters that caused the difference between results shown in Figure 3.6 and BPA results shown in Figure 3.8.

\subsection{Real-time Security Assessment Application}

The procedures of simulations have been automatically implemented in SimAuto. Besides saving lots of simulation effort, this opens up the potential of assessing operation condition in real time. The framework of transitional security assessment is shown in Figure 3.14. The season nomogram created in this way is very conserve because it is calculated based on the worst scenario. The measurements from Phasor Measurement Units (PMUs) are collected and compared against the seasonal nomogram for online security assessment. Alternatively, the system operation condition can be updated periodically and the real-time nomogram can be adjusted accordingly (see Figure 3.15). In this way, the nomogram only needs to cover a variety of operating conditions that are most likely in the short term. Therefore, this provides more accurate restriction on the system operation conditions, and results in the more efficient utilization of transmission capacity. 


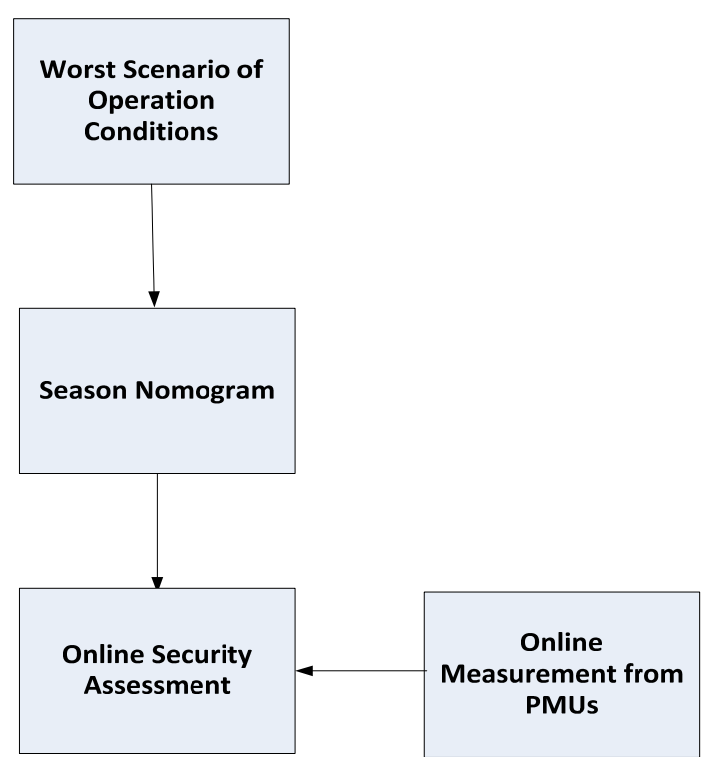

Figure 3.14. Convention Online Security Assessment

A new software has been developed for the real-time security assessment using the nomograms that have been built, as shown in Figure 3.16. It essentially accomplishes three objectives: express-analysis of the available security margin, determining the most limiting elements of the grid, and selecting remedial actions. The main display screen is divided into three parts: the three-dimensional view of the closest hyperplane shown in the left panel, security margin displayed in the right upper panel, and remedial action recommended to mitigate instability risks given in the right bottom panel. Figure 3.16 shows how it can be used when the operation condition is changing. When the operation condition is within a safe distance from the hyperplane, the security margin is more than 100-MW. When it moves toward the security boundary, the security margin shrinks to negative value. The right bottom part of the screen shows the sensitivities of area generation with respect to the security margin, which implies how the area generations or loads are changed to improve the security margin.

\subsection{Evaluate Transformation of Hyperplanes from Power Flow Space to Angle Space}

A preliminary study (see Appendix C) has been conducted to evaluate the transformation of hyperplanes from power flow space to angle space. 


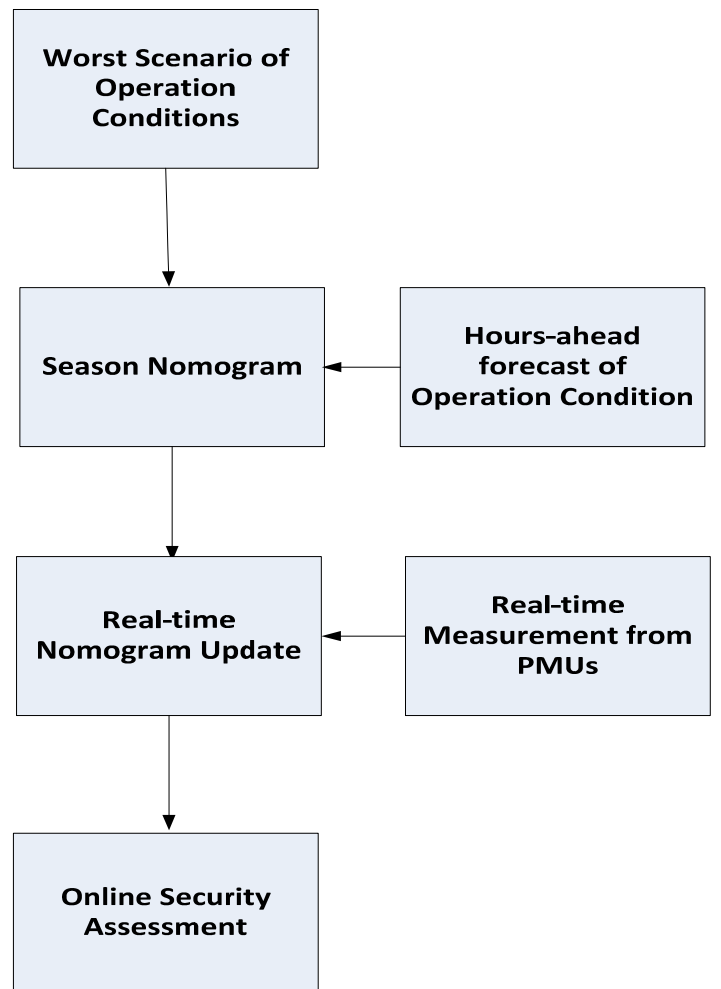

Figure 3.15. Proposed Online Security Assessment 

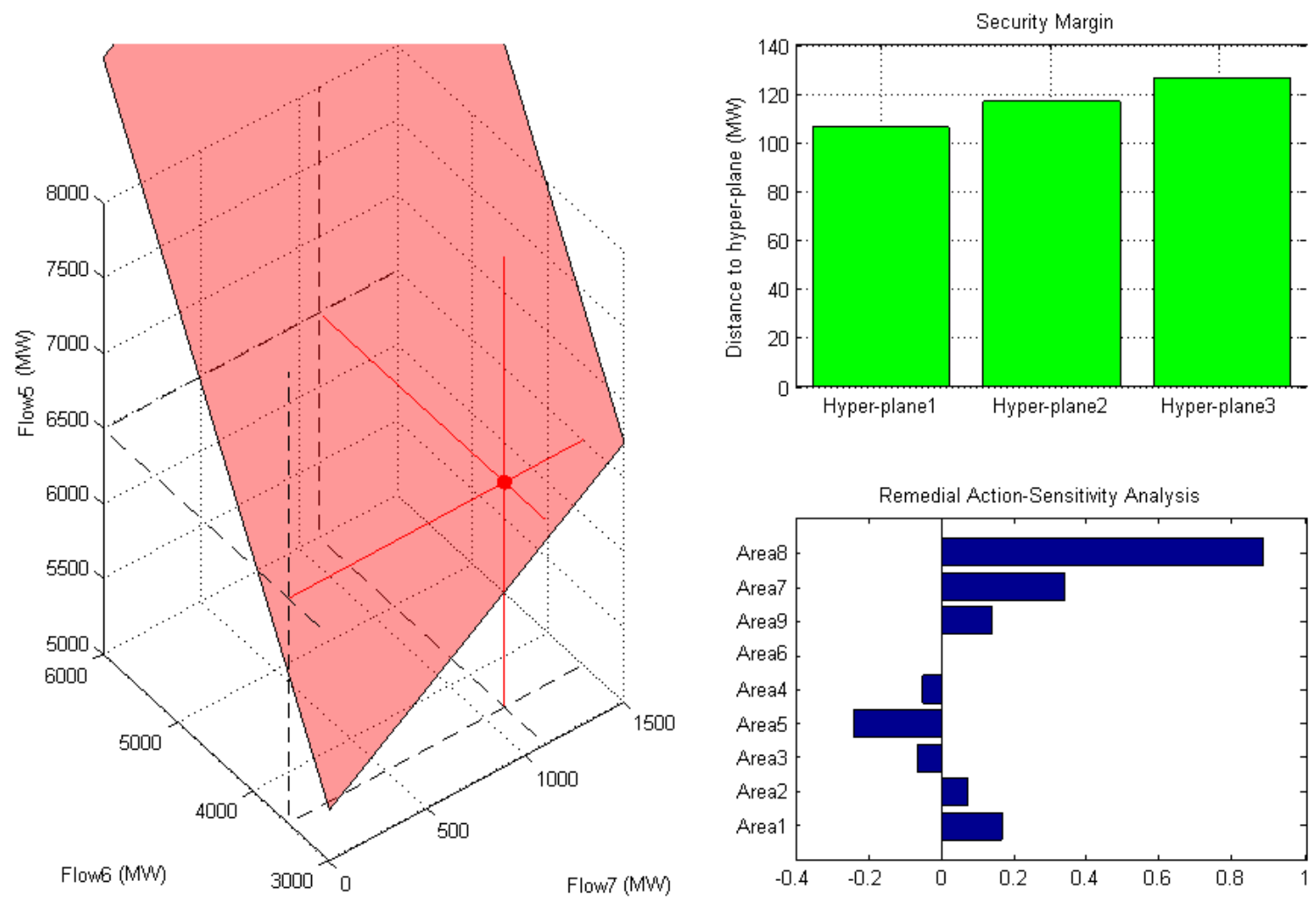

(a)Operation Point in the Safe Distance from Hyperplanes
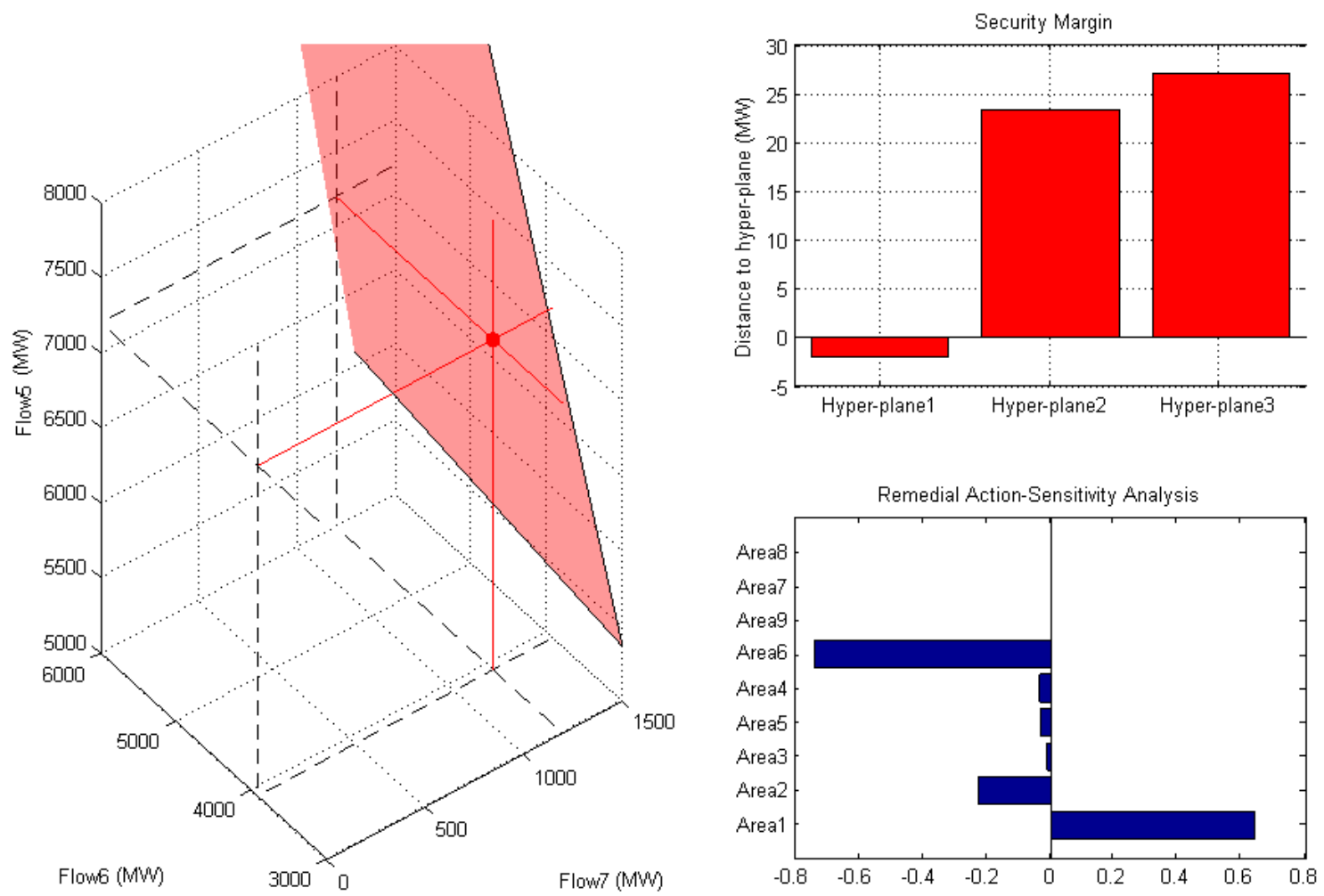

(b) Operation Point Moving toward Hyperplanes

Figure 3.16. Real-time Application of Hyperplanes to Monitor Stability Margin 


\subsection{Conclusions and Future Work}

This project has developed methodologies for power system security assessment based on the idea of wide-area multidimensional security region and piecewise linear approximation of nonlinear security boundaries. The procedures to determine the boundary points and find the security hyperplanes in the multidimensional parameter space have been described. Numerical simulations were conducted on the WECC system. Numerical examples demonstrated the validity of the proposed approach and its capability of quantifying power transfer limits and security margins. The comparison between the hyperplanes built and the existing nomograms shows that the proposed approach, by considering more system variables at the same time, is less conservative and more accurately represents the system's security boundary.

Major achievements of the project are summarized as follows.

1. Critical control parameters (area loads and generations) and descriptor parameters (path flows) essential for security analysis are identified in the Pacific Northwest power system.

2. Practical methodologies and procedures have been proposed to build hyperplanes piecewiselinearly approximating the wide-area security region of electrical power systems, taking into consideration all types of constraints: thermal, voltage, voltage stability, transient and oscillatory stability limits. The compromise between accuracy and computational cost is achieved by selecting a minimum set of hyperplanes describing the boundary with a given accuracy.

3. Methodologies are also developed to quantify security margins, identify weak elements in the system, and calculate key contributing factors and sensitivities to assist in determining remedial actions.

4. Numerical simulations have been carried out on WECC system using 2007 heavy summer base case. 92 hyperplanes have been built in a selected feasible area of the 8-dimension descriptor parameter space, based on WECC criteria and BPA margin requirements.

5. The simulation procedures implemented in Matlab and PowerWorld SimAuto can be performed automatically. There is great potential that the proposed methodology can be applied in the real-time operations by: (1) recalculating security hyperplances based on updated system configurations and (2) assessing security margins and finding optimal control strategies using the updated hyperplanes.

6. A prototype operational tool for online security assessment is built in Matlab to demonstrate the use of multidimensional security hyperplanes and real-time security margin assessment and optimal control direction calculation.

7. The transformation of security hyperplanes between the power flow space and phasor angle space is investigated preliminarily.

The future work being planned includes: 
1. Combine offline computer simulations run for the system model with real-time power system measurement, including SCADA and state estimation data, and subsecond PMU data for a quick evaluation of the real-time system security margin.

2. Evaluate different options (such as generator drops, load shedding, capacitor switching, etc.) to increase the security margin. These options can be incorporated into the framework proposed to evaluate their values, and serve as advisory tool to operators.

3. Refine the procedures developed to build security hyperplanes across the entire control and description parameter space and validate the resulting hyperplanes built for the BPA power system. The resulting hyperplane sets could be used by BPA in parallel with the existing nomograms initially. With the increasing confidence in the newly proposed methodology, it can be later applied to refine the existing set of nomograms and transmission limits.

4. Further develop the automated simulation program for building offline security hyperplanes to increase the degree of automation in the entire process to include not only thermal, voltage and voltage stability limits, but also oscillatory and transient stability limits.

5. Further develop functionalities of the operational tool that apply security constraints obtained by offline computer simulations to real-time SCADA and subsecond phasor measurement unit data, for online security assessment. For example, when calculating optimal control directions, consider all types of control capabilities in the system instead of only area load and generation, feasibility of the control direction under the particular operating condition, and operator preferences.

6. Develop a specification for building an industrial-grade tool out of the methodologies and procedures developed in the project.

7. Use the methodology to benchmark system behavior based on phasor measurements (which is the highest priority for NASPI).

\section{Provide technology transfer to BPA.}

Significance of the work includes the following:

1. More accurate description of security region minimizes system redispatch cost caused by security constraints and increases utilization of the transmission grid.

2. Increases system reliability by real-time monitoring of system security for constraints (thermal, voltage, transient stability, voltage stability, and others).

3. Provides instantaneous actionable information helping to identify best combination of controls increasing the security margin.

4. Security boundary description in the form of linear inequalities directly fits into the Security Constrained Unit Commitment and Security Constrained Unit Dispatch software.

5. Expected to be easily accepted by the industry because it is a logical expansion of the existing security nomograms. 


\subsection{References}

[1] F Wu and S Kumagai. 1982. "Steady-State Security Regions of Power Systems." IEEE Trans. on Circuits and Systems 29(11):703-711.

[2] R Kaye and F Wu. 1982. "Dynamic Security Regions of Power Systems." IEEE Trans. on Circuits and Systems 29(9):612-623.

[3] YV Makarov, P Du, TB Nguyen, and S Lu, Wide Area Power System Security Region Intermediate Report. Pacific Northwest National Laboratory, November 2008.

[4] J Su, Y Yu, H Jia, P Li, N He, Z Tang, and H Fu. 2002. "Visualization of Voltage Stability Region of Bulk Power System." Proceedings of International Conference on Power System Technology PowerCon 2002, Vol. 3, October 13-17, 2002, pp. 1665-1668.

[5] ST Lee. 2003. "Community Activity Room as a New Tool for Transmission Operation and Planning Under a Competitive Power Market." Proceedings of 2003 IEEE Bologna Power Tech Conference, Vol. 4, June 23-26, 2003.

[6] PA Dolloff, D Adams, S Anderson, MY Vaiman, MM Vaiman, M Povolotskiy, and DT Bradshaw. 2008. "Evaluate Real-time System Reliability Using Boundary-based Concept for EKPC System." Proceedings of the 2008 PES General Meeting, Paper PESGM2008-001455, July 20-24, 2008.

[7] P Li, Y Yu, H Jia, J Su, and J Zhao. 2002. "A Study on Boundary of Small Disturbance Stability Region." Proceedings of International Conference on Power System Technology PowerCon 2002, Vol. 2, October 13-17, 2002, pp. 1228-1232.

[8] Y Zeng and Y Yu. 2002. "A Practical Direct Method for Determining Dynamic Security Regions of Electrical Power Systems." Proceedings of International Conference on Power System Technology PowerCon 2002, Vol. 2, October 13-17, 2002, pp. 1270-1274.

[9] Y Zeng, Y Yu, and H Jia. 2005. "Theoretical Explanation of Hyper-Plane Boundary of Dynamic Security Region." Proceedings of Canadian Conference on Electrical and Computer Engineering, May 1-4, 2005, pp. 1946-1949.

[10] C Dong and Y Yu. 2005. "PDSR in Phase Angle Space and SPM Based Security Monitoring of Power Systems." IEEE Region 10 TENCON 2005, November 2005, pp. 1-5.

[11] Y Yu, Y Zeng, C Huang, ST Lee, and P Zhang. 2004. “A Practical Direct Method for Determining Dynamic Security Regions of Electrical Power Systems by Power Perturbation Analysis."

Proceedings of International Conference on Electrical Engineering ICEE2004, July 4-8, 2004, Sapporo, Japan.

[12] M Parashar, Electric Power Group; A Agarwal, Electric Power Group; Y Makarov, Pacific Northwest National Laboratory; and I Dobson, University of Wisconsin, Madison. December 2007. "Real Time Voltage Security Assessment (RTVSA) Algorithms \& Framework.” Prepared for California Independent System Operator by Consortium for Electric Reliability Technology Solutions, funded by California Public Interest Energy Research Transmission Research Program, Contract No. 500-02-004, MR-041. Available at http://certs.lbl.gov/certs-rt-pubs.html.

[13] IA Hiskens and RJ Davy. 2001. "Exploring the Power Flow Solution Space Boundary." IEEE Power Engineering Review 21(8):57-57.

[14] I Dobson. 1992. "Observations on the Geometry of Saddle Node Bifurcation and Voltage Collapse in Electrical Power Systems." IEEE Trans. on Circuits and Systems I: Fundamental Theory and Applications 39(3):240-243. 
[15] MM Begovic and AG Phadke. 1992. "Control of Voltage Stability Using Sensitivity Analysis." IEEE Trans. on Power Systems 7(1):114-123.

[16] I Dobson and L Lu. 1993. "New Methods for Computing a Closest Saddle Node Bifurcation and Worst Case Load Power Margin for Voltage Collapse." IEEE Trans. on Power Systems 8(3):905-913.

[17] M Djukanovic, DJ Sobajic, and Y-H Pao. 1993. "Learning Tangent Hypersurfaces for Fast Assessment of Transient Stability." Proceedings of the Second International Forum on Applications of Neural Networks to Power Systems ANNPS '93, April 19-22, 1993, pp. 124-129.

[18] PA Dolloff, D Adams, S Anderson, MY Vaiman, MM Vaiman, M Povolotskiy, and DT Bradshaw. 2008. "Evaluate Real-time System Reliability Using Boundary-based Concept for EKPC System." Proceedings of the 2008 PES General Meeting, Paper PESGM2008-001455, July 20-24, 2008.

[19] M Parashar, Electric Power Group; A Agarwal, Electric Power Group; Y Makarov, Pacific Northwest National Laboratory; and I Dobson, University of Wisconsin, Madison. 2008. "RealTime Voltage Security Assessment (RTVSA): Summary Report." Prepared for California Independent System Operator by Consortium for Electric Reliability Technology Solutions, funded by California Public Interest Energy Research Transmission Research Program, Contract No. 500-02-004, MR-041, January 21, 2008. Available at: http://certs.lbl.gov/pdf/task3-rtvsasummary-rpt.pdf.

[20] JD McCalley, S Wang, Q-L Zhao, G-Z Zhou, RT Treinen, and AD Papalexopoulos. 1997. "Security Boundary Visualization for Systems Operation." IEEE Trans. on Power Systems 12(2):940-947.

[21] YV Makarov, DJ Hill, and Z-Y Dong. 2000. "Computation of Bifurcation Boundaries for Power Systems: A New $\Delta$-Plane Method." IEEE Trans. on Circuits and Systems I: Fundamental Theory and Applications 47(4):536-544.

[22] YV Makarov, DJ Hill, and IA Hiskens. 2000. "Properties of Quadratic Equations and Their Application to Power System Analysis.” Electrical Power and Energy Systems 22:313-323.

[23] PW Sauer, K Tomsovic, J Dagle, S Widergren, T Nguyen, and L Schienbein. 2004. "Integrated Security Analysis.” Final Report, CERTS, July 2004. Available online: http://certs.lbl.gov/CERTS_P_RealTime.html.

[24] MA El-Sharkawi, RJ Marks, ME Aggoune, DC Park, MJ Damborg, and LE Atlas. 1989. "Dynamic Security Assessment of Power Systems Using Back Error Propagation Artificial Neuron Networks." Proceedings of the 2nd Symposium on Expert System Applications to Power Systems, Seattle, Washington, July 1989.

[25] R Kumar, A Ipahchi, V Brandwajan, MA El-Sharkawi, and G Cauley. 1991. "Neuron Networks for Dynamic Security Assessment of Large Scale Power Systems: Requirements Overview." Proceedings of 1st International Forum on Applications of Neuron Networks to Power Systems, Seattle, Washington, July 1991, pp. 65-71.

[26] AA EI-Keib and X Ma. 1995. "Application of Artificial Neural Networks in Voltage Stability Assessment." IEEE Trans. on Power System 10(4):1890-1896.

[27] S Chauhan and MP Dava. 1997. "Kohonen Neural Network Classifier for Voltage Collapse Margin Estimation." Electric Machines and Power Systems 25(6):607-619.

[28] Y Mansour, AY Chang, J Tamby, E Vaahedi, BR Corns, and MA El-Sharkawi. 1997. "Large Scale Dynamic Security Screening and Ranking Using Neuron Networks." IEEE Trans. on Power Systems 12(2):954-960. 
[29] HB Wan and YH Song. 1998. "Hybrid Supervised and Unsupervised Neural Network Approach to Voltage Stability Analysis." Electric Power System Research 47(2):115-122.

[30] VR Dinavahi and SC Srivastava. 2001. "ANN Based Voltage Stability Margin Prediction.” IEEE Power Engineering Society Summer Meeting 2001 Vol. 2, July 15-19, 2001, pp. 1275-1280.

[31] A Sittithumwat and K Tomsovic. 2002. "Dynamic Security Margin Estimation Using Artificial Neural Networks." IEEE Power Engineering Society Summer Meeting 2002, Vol. 3, pp. $1322-1327$.

[32] MM Salamaa, EM Saieda, MM Abou-Elsaada, and EF Gharianyb. 2001. "Estimating the Voltage Collapse Proximity Indicator Using Artificial Neural Network." Energy Conversion and Management 42(1):69-79.

[33] H Hakim. 1992. "Application of Pattern Recognition in Transient Security Assessment." Journal of Electrical Machines and Power Systems, 20:1-15.

[34] L Wehenkel. 1998. Automatic Learning Techniques in Power Systems. Kluwer Academic Publishers, Massachusetts.

[35] C Aldea and SC Savulescu. 2004. "Evaluation of the Stability Reserve of Transelectrica's Transmission System by Using Quickstab Professional." WEC Regional Energy Forum - FOREN 2004, Neptun, June 13-17, 2004.

[36] P Dimo. 1971. “L’Analyse Nodale des Réseaux D’Énergie.” Eyrolles.

[37] "WECC System Performance Criteria", Apr. 18, 2008, WECC. [Online.] Available: http://wecc.biz/standards/wecc\%20criteria/forms/allItems.aspx 



\section{Appendix A}

Literature Review on the Security Region Concept for Power Systems 



\section{Appendix A}

\section{Literature Review on the Security Region Concept for Power Systems}

This section describes the recent advances in developing the security region concept and discusses their implications and future perspective.

\section{A.1 Methods}

Selection of the performance measures is dependent on the type of security problem being studied. Typical performance measures for the most common security problems are given below:

1. Thermal overload: amperes or MVA on the overloaded circuit; percent of violation with respect to the rating.

2. Voltage out of limits: voltage magnitude at the violated bus; percent of violation with respect to the nominal voltage.

3. Voltage instability: MVAR or MW "distance" to the bifurcation point, e.g., the distance to the "nose" point of a QV or PV curve or distance to the closest bifurcation point in the multidimensional space.

4. Transient instability: voltage dip, frequency dip, energy margin, or critical fault clearing time.

5. Oscillatory instability: damping ratio or the real part of the dominant eigenvalue corresponding to a potentially unstable mode.

\section{A.1.1 Selection of Critical Parameters}

A critical parameter, selected for a specific contingency and related security problem, is a parameter that serves as a good predictor of the post contingency performance measure. The critical parameter set must satisfy the following criteria:

1. Set Sufficiency: The parameters must contain sufficient information to allow prediction of the post-contingency performance measure within a desired accuracy for all operating conditions within the study scope.

2. Set Cardinality: The critical parameter set should be chosen as the set of minimum size which satisfies the set sufficiency criterion.

3. Operating Point Controllability: At least one critical parameter within the set must be controllable by the operator so that the operating point can be adjusted, with respect to the boundary, using preventive actions. 


\section{A.1.2 Steady-state Security Region [1],[8],[13]}

The steady-state security region determines a set of load demand and generation values, for which all the equipment is loaded within its allowable constraints, and where the power flow solutions exist. Usually, it is evaluated for N-1 and some N-2 contingency conditions. Security constraints may include:

1. Voltage magnitude constraints;

2. Line current constraints;

3. Real power generation constraints;

4. Reactive power generation constraints.

In [1], decoupled power flow equations were used to determine the steady-state security region $R_{Z}$ with respect to a set of given contingencies. The assumed features of $R_{Z}$ are

1. $R_{Z}$ lies on or above a set of supporting hyperplanes;

2. $R_{Z}$ is convex.

In a deterministic approach, all contingencies are treated equal regardless of how likely they are to occur. EPRI developed a new performance index based on these three types of reliability impacts and combined them with probabilistic factors that correctly and consistently recognize the different contingencies that may cause these impacts. The Community Activity Room (CAR) is a concept to analytically describe and visualize a system security region in the state space of net inter-regional power exports [8]. The concept is illustrated in Figure A. 1. Based on a set-theoretic approach, its basic idea is to use the net MW power injections at each bus as the independent variables, and express the line flow in terms of these variables. The key performance measures of a transmission grid are thermal overloads, low voltages, and voltage collapse or dynamic instability.

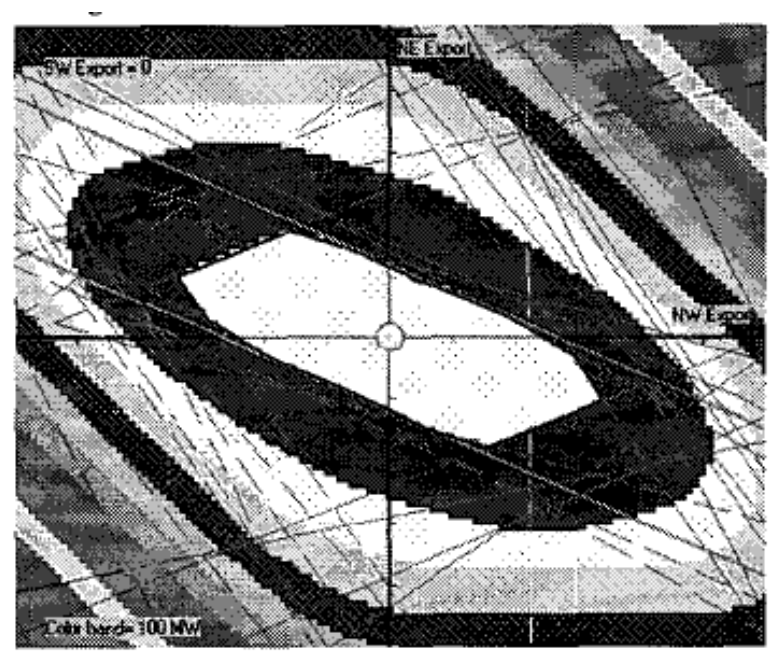

Figure A. 1. Probabilistic Community Activity Room 


\section{A.1.3 Small-Disturbance Stability Regions (SDSR) [7]}

Small signal stability of power systems refers to the ability of the system to remain close to a stable equilibrium after a small disturbance. The disturbance should be small enough so that the system can be linearized around that equilibrium point for analysis. The conventional method of small disturbance stability analysis is using eigenvalues of the linearization around an operation point the differentialalgebraic equations (DAE) used to model the systems. SDSR is bounded by three kinds of local bifurcation points, saddle-node bifurcation point (SNB), Hopf bifurcation point (HB), and singular induced bifurcation point (SIB).

In [7], SDSR is defined in parameter space, and it consistes of all operating points that can be reached from a given operating point by continuous variations of parameters without loss of small disturbance stability. A modified Philips-Heffron model of generator is derived for a static load and induction motor load. Generator is modeled as fifth order model accounting for dynamics o $\mathrm{f} E_{q}^{\prime \prime}, E_{q}^{\prime}, E_{d}^{\prime \prime}$ and AVR is assumed to separately excited fast excitation system. Figure A. 2 shows the SDSR boundary with different induction load percentage.

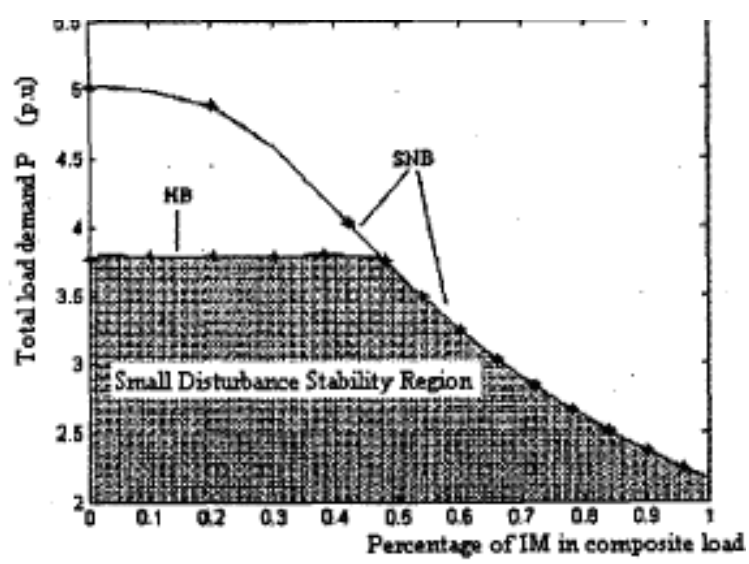

Figure A. 2. SDSR's Boundary Versus Percentage of Induction Motor

\section{A.1.4 Voltage Stability}

Voltage stability has become a major issue for the secure operation of power systems around the world. It can be divided into small-disturbance (SD) voltage stability and large-disturbance (LD) voltage stability.

Large-disturbance voltage stability deals with the system's ability to maintain voltages after such disturbances as generation trips, load loss, and system faults. It is analyzed by modeling long-term system dynamics. Large-disturbance voltage stability is analyzed by solving a set of nonlinear differential or algebraic equations (time domain simulations or numerical solution). The system is considered voltage stable if its post-transient voltage magnitudes remain limited by certain pre-established reliability limits (5-10\% depending on the severity of disturbance). 
Small-disturbance stability is concerned with the ability of the system to control voltages following small perturbations or gradual change of parameters such as system load. This type of steady-state stability is analyzed by linearizing nonlinear differential equations at a given operating point. The voltage collapse can be explained as a dynamic consequence of a saddle node bifurcation instability in which the system operating equilibrium disappears.

The standard power flow can be expressed as

$$
f(x, \lambda)=0
$$

where $x \in R^{n}, \lambda \in R^{p}$, and $f: R^{n+p} \rightarrow R^{n}$. As is well-known, a maximum loading point satisfies the following power-flow-singularity condition

$$
\left|f_{x}(x, \lambda)\right|=0
$$

where $f_{x}(x, \lambda)$ is the Jacobian matrix of $f$ with respect to $x$. The set $\Sigma$ of the maximum loading points can be defined as follows:

$$
\Sigma=\left\{(x, \lambda)|f(x, \lambda)=0,| f_{x}(x, \lambda) \mid=0\right\}
$$

Figure A. 3 illustrates the power flow solution curves for different values of $\lambda$.

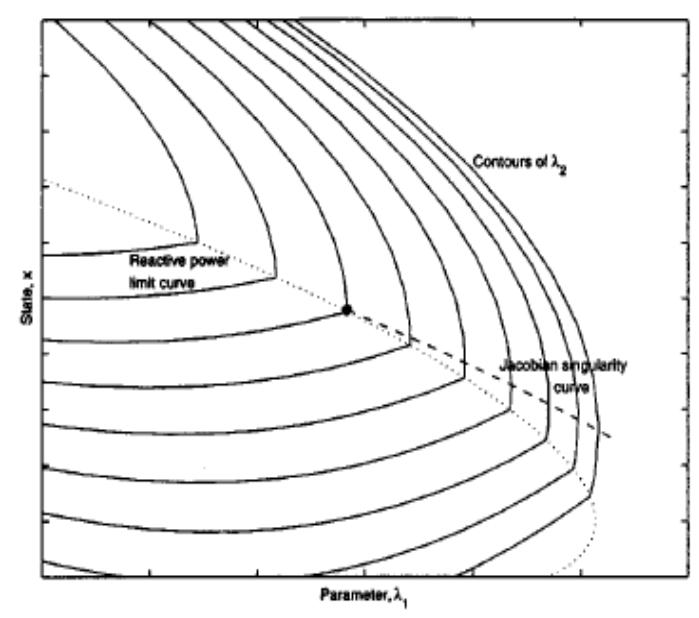

Figure A. 3. Power Flow Solution Curves

\section{A.1.5 Predictor-corrector Approach}

Parameter continuation predictor-corrector methods are the most reliable power flow methods capable of reaching the point of collapse on the power flow feasibility boundary. The addition of new variables, called continuation parameters, determines the position of an operating point along some power system stress direction in the parameter space. The predictor step consists of an incremental movement of the power flow point along the state space trajectory, based on the linearization of the model. The corrector step, which follows each predictor step, consists in the elimination of the linearization error by balancing 
the power flow equations to some close point on the nonlinear trajectory. The main steps of the algorithm are illustrated in Figure A. 4.

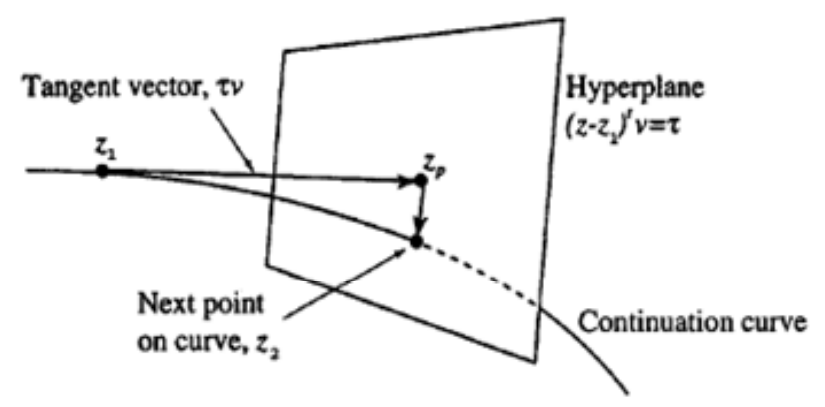

Figure A. 4. Predictor-corrector Process

Points on the power flow solution space boundary are described by

$$
\begin{gathered}
f(x, \lambda)=0 \\
f_{x}(x, \lambda) y=0 \\
y^{t} y=1
\end{gathered}
$$

a set of $2 n+1$ equations in $2 n+p$ unknowns. It is convenient to write this as a general set of equations

$$
\phi(z)=0
$$

where $z=\left[\begin{array}{l}x \\ y \\ \lambda\end{array}\right] \in R^{2 n+2}, \phi=\left[\begin{array}{c}f \\ f_{x} y \\ y^{t} y-1\end{array}\right]: R^{2 n+2} \rightarrow R^{2 n+1}$.

\section{Algorithm:}

\section{Prediction Step:}

The unit vector $v \in R^{2 n+2}$ that is tangent to the feasible boundary at $z_{1}$ is given by

$$
\begin{gathered}
\left.\phi_{Z}\right|_{Z=Z_{1}} v=0 \\
\|v\|=1
\end{gathered}
$$

where $\phi_{Z}$ is the $(2 n+1) \times(2 n+2)$ Jacobian $\partial \phi / \partial z$. The prediction of the next point on the curve is

$$
z_{p}=z_{1}+\tau v
$$

where $\tau$ is a scalar control parameters.

\section{Correction Step:}


The Euler method is used to correct to a point $z$ on the curve by solving for the point of intersection of the curve and a hyperplane that passes through $z_{p}$ and that is orthogonal to $v$. The point of intersection of the curve and the hyperplane is then given by

$$
\begin{gathered}
\phi(z)=0 \\
\left(z-z_{p}\right)^{t} v=0
\end{gathered}
$$

Together, (Equation (A.8)) and (Equation (A.9)) form a set of $2 n+2$ equations in $2 n+2$ unknowns. They can be solved using a standard technique such as Newton-Raphson.

\section{A.1.6 Direct Method}

Direct methods for finding the point of collapse (PoC) in a given direction combine a parametric description of the system stress, based on the specified loading vector in the parameter space and a scalar parameter describing a position of an operating point along the loading trajectory and the power flow singularity condition expressed with the help of the Jacobian matrix multiplied by a nonzero right or the left eigenvector that nullifies the Jacobian matrix at the collapse point. Unlike the power flow problem, this reformulated problem does not become singular at the point of collapse and can produce the bifurcation point very accurately.

\section{Algorithm:}

The problem is formulated as

Maximize [Total MVA demand]

Subject to

1. Distribution constraints at load buses

2. MVAR and MW limits on generators

3. Generators MW participations

4. Constant power factor of MVA demand

5. Limits on control voltages and LTC transformer taps

The variables of this optimization problem are voltage angles at all buses except the slack bus, voltage magnitude, LTC transformer taps, and generator participation factors. 


\section{A.2 Case Studies}

\section{A.2.1 Visualization of Voltage Stability Region of Bulk Power System [5]}

Yu proposed the visualization of the feasible region in the critical cut-set space. After the weak nodes are identified, CPF (Continuation Power Flow) is used to construct the geographical representation of hyperplanes. The main steps of the algorithms are as follows:

1. Find the weak node set of system with $L_{i}$ index (or modal analysis);

2. Define the critical cut-set with $\mathrm{N}$ branches according to voltage stability constraints, as illustrated in Figure A. 5;

3. Apply CPF method, search out at least $2 \mathrm{~N}$ branch transmission critical points of critical cut-set;

4. Using LSM, approximate hyperplane as system voltage stability boundary, as illustrated in Figure A. 5

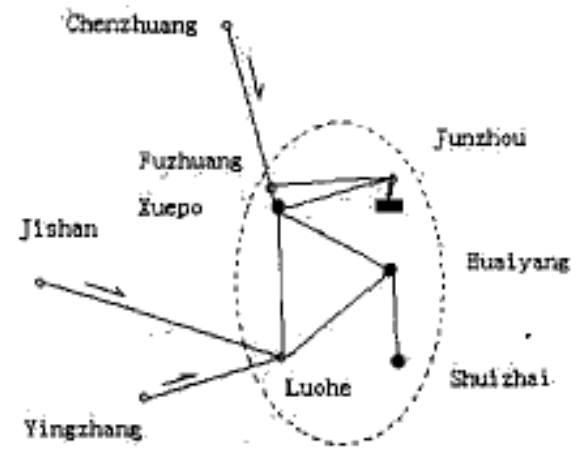

Figure A. 6. Critical Cut-set Identification (the weak nodes are mostly located on the terminal of long transmission lines without reactive power support)

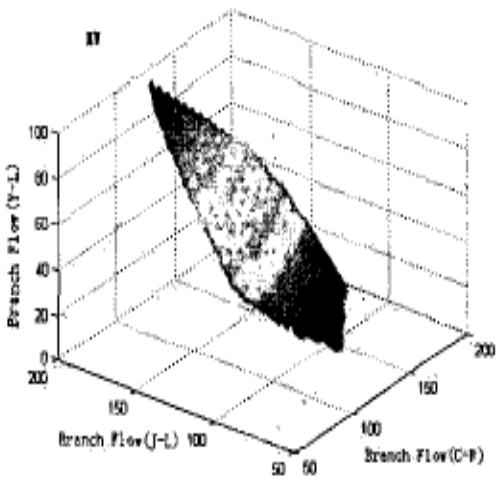

(a)

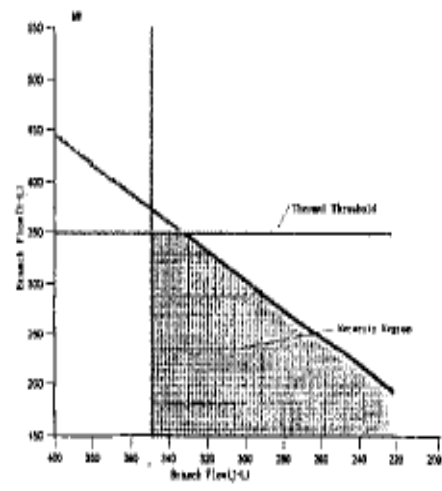

(b)

Figure A. 7. Voltage Stability Boundary (hyperplane) (a: stability boundary in critical cut-set space; b: 2-dimension feasible region with thermal and voltage stability limits) 


\section{A.2.2 Boundary-based Concept for EKPC System [9]}

Physical and Operational Margins (POM), which is developed by V\&R Energy system research, is contingency analysis program in which four constraints are considered:

1. Voltage stability,

2. Thermal overload of lines and transformers,

3. Flow gate limits,

4. Voltage violation (voltage range and/or pre-contingency to post-contingency voltage drop).

This software package is used to identify the boundary of the secure operating region for each contingency for East Kentucky Power Cooperative. A total of 3,736 N-1 contingencies were applied during this simulation on the power system network model consisting of 8,777 buses and 26,215 branches. Out of 3,736 N-1 contingencies, 141 contingencies (less than 4\%) were identified as critical. The boundary of the operating region may be shown as a projection onto different planes, such as power injections, load and generation, interface, and/or tie-line flows. The effect of contingencies on the size and the shape of the operating region and on the limiting constraints was also identified. A typical secure operating region for the base case condition (e.g., N-0 condition) is shown in Figure A. 8.

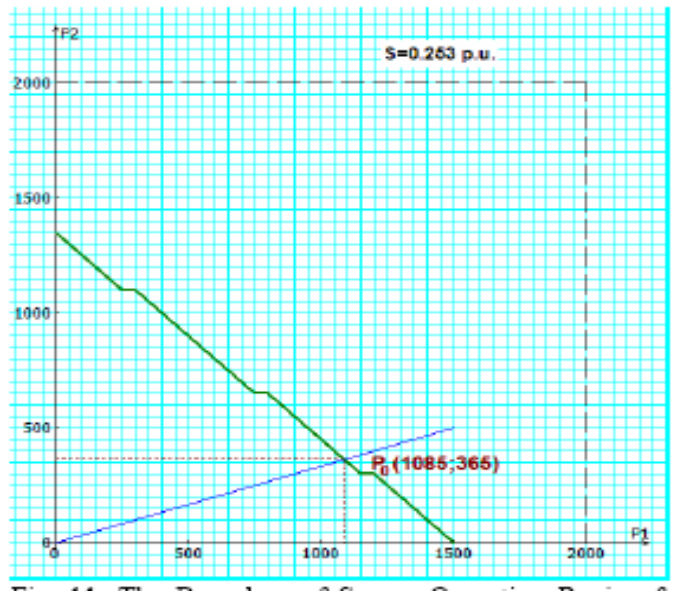

Figure A. 9. Boundary of Secure Operating Region for Two Simultaneous Transfers

Upon determining effective mitigation measures to alleviate post-contingency voltage and thermal violations, the voltage stability margin for each of 3,736 N-1 contingencies was computed to assess the vulnerability of the power system network. To increase the maximum transfer capability, four mitigation measures were utilized, in the following order of priority:

1. MVAr redispatch

2. Transform tap change

3. Capacitor and reactor switching

4. Line switching 


\section{A.2.3 Real-time Voltage Security Assessment (RTVSA) [1]}

RTVSA is a variation of the predictor-corrector type of the continuation power flow. At the Point of Collapse, the left eigenvector of the power flow Jacobian matrix is calculated to estimate the tangent hyperplane of the load flow feasibility boundary. Procedures have been developed to select the minimum set of cut set planes to approximate the power flow feasibility boundary. If the feasibility boundary is smooth and convex, the hyperplane building can conservatively represent the secure operating region. The flow chart of the algorithm is shown in Figure A. 10.

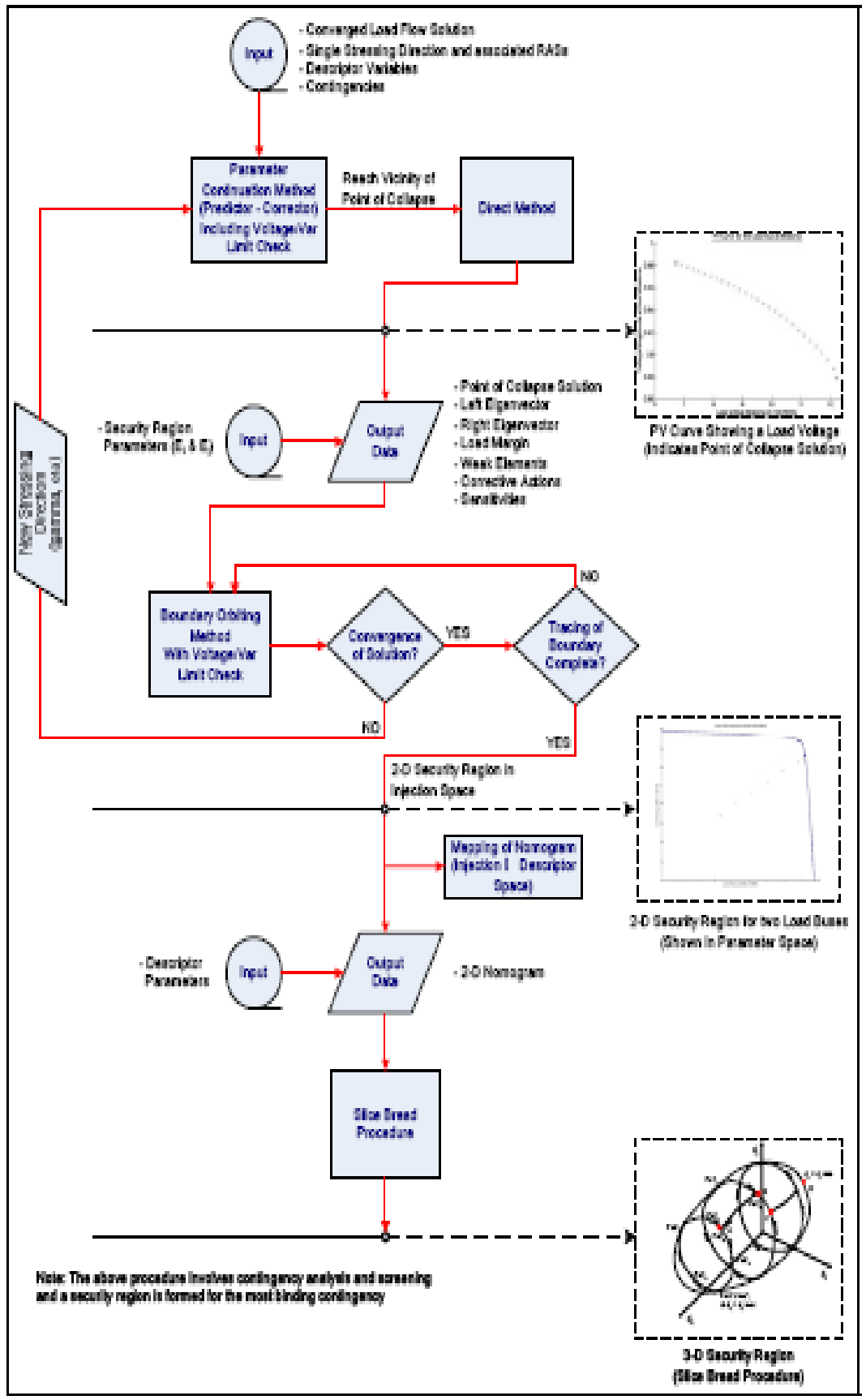

Figure A. 11. RTVSA Algorithms Flowchart 


\section{A.3 Voltage Stability Margin and Sensitivity Analysis}

In [15], the minimum singular value was proposed to determine the stability margin. However, discontinuities observed in the minimum singular value are to the results of the Q-limits reached by some generators. In fact, the Q-limits create structural changes in the Jacobian matrix. In addition, they cause the bifurcation point to occur at a lighter load condition. All these make the minimum singular value a poor indicator to voltage stability.

Ian Dobson computed the first-order sensitivities of a load power margin index to controls or parameters appearing in the load flow equations [16]. Suppose $\Sigma$ consists of curved hyper-surfaces of the secure operating region, and the current control parameters are denoted by $\lambda_{0}$. The closest bifurcation point to $\Sigma$ is $\lambda_{*}$ at which the system loses stability. Therefore, the proximity to voltage collapse can be measured by the distance $\left|\lambda_{*}-\lambda_{0}\right|$. The gradient with respect to $\lambda$ of the index $\left|\lambda_{*}-\lambda\right|$ evaluated at $\lambda_{0}$ is $\left.\nabla_{\lambda}\left(\left|\lambda_{*}-\lambda\right|\right)\right|_{\lambda_{0}}$. This is the sensitivity of the load power margin with respect to control and its direction gives the optimum direction for control. $\left.\nabla_{\lambda}\left(\left|\lambda_{*}-\lambda\right|\right)\right|_{\lambda_{0}}$ is parallel to the normal vector $n_{*}$ to $\Sigma$ at $\lambda_{*}$.

\section{A.4 Transient Stability}

There are three main approaches to the transient stability assessment problem.

Classically, the transient stability analysis is carried out through a series of system simulations in a broad range of situations. One of the advantages of the time domain simulation method is its unlimited modeling capability. Despite its advantages, the time domain simulation method has two shortcomings. Firstly, it is inherently slow because of integration process involved in solving the differential equations. Secondly, it does not yield the information on the system's degree of stability.

The energy concept and the equal area criteria were exploited to estimate the region of stability or attraction area of power systems. They can directly provide stability information without solving the differential equations. However, they still have the limited modeling capability, and address the power system stability only up to the first swing after disturbance. The hybrid combines both the time domain method and the transient energy function evaluation for power system transient stability analysis. It first computes the system trajectory using time-domain simulation, then calculates the degree of stability by evaluating the transient energy function.

Artificial Intelligence can be used as an effective approach for transient stability assessment. To make the overall transient stability assessment problem tractable, this approach decomposes it into elementary problems. An elementary transient stability assessment problem is defined with respect to a given network topology and contingency. For such an elementary problem, Artificial Intelligence builds decision rules off-line using a large number of examples (Learning Set). These are operating points whose stability class is known a priori. On-line assessment involves retrieving decision rules for the given operating condition. 


\section{A.5 Direct Method and Energy Function}

The stability boundary of nonlinear autonomous dynamical systems is described by

$$
\dot{x}=f(x)
$$

where the vector field $f$ is $C^{1}$.

The solution curve of (Equation (A.10)) starting from $x$ at $t=0$, denoted by $\Phi(x, \cdot): R \rightarrow R^{n}$, is called a trajectory. Suppose that $x_{s}$ is a (asymptotically) stable equilibrium point (s.e.p.) of the system, then there is a region $A\left(x_{s}\right)$ contains $x_{s}$ such that every trajectory in this region converges to the s.e.p. $x_{s}$ as time increases. The stability boundary (the boundary of stability region) is denoted by $\partial A\left(x_{s}\right)$. An illustration is shown in Figure A. 12.

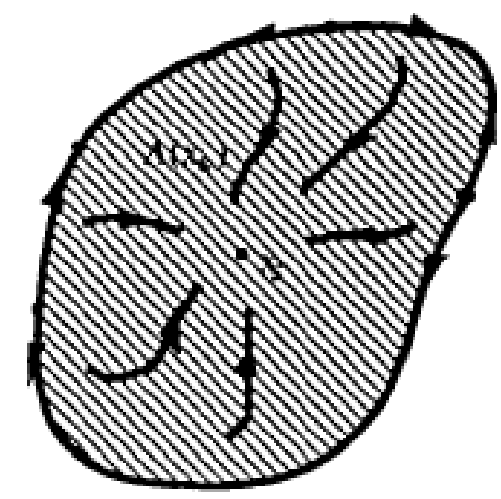

Figure A. 13. The Boundary of Stability Region

A function $V: R^{n} \rightarrow R$ is said to be an energy function if it satisfies the following three conditions:

1. $\dot{V}(\Phi(x, t)) \leq 0$ at $x \notin E$

2. If $x$ is not an equilibrium point, then the set $\{t \in R: \dot{V}(x(t))=0\}$ has measure zero in $R$

3. If $V(\Phi(x, t))$ is bounded in positive time, then $\Phi(x, t)$ is bounded in positive time.

The first two properties imply that energy functions are strictly decreasing along system trajectories. The third property is import in the characterization of stability boundary of system. As time increases, every trajectory on the stability boundary $\partial A\left(x_{s}\right)$ converges to one of the equilibrium points on $\partial A\left(x_{s}\right)$ and every trajectory in the stability region $A\left(x_{s}\right)$ converges to the s.e.p. This result provides a sufficient condition for stability. 


\section{A.5.1 System Model and Energy Function}

For the system model being considered, the equations of motion are

$$
\begin{gathered}
M_{i} \dot{\omega}_{i}=P_{i}-P_{e i} \\
\dot{\delta}_{i}=\omega_{i} \quad i=1, \cdots n
\end{gathered}
$$

where $P_{e i}=\sum_{\substack{j=1 \\ \neq i}}\left[C_{i j} \sin \left(\delta_{i}-\delta_{j}\right)+D_{i j} \cos \left(\delta_{i}-\delta_{j}\right)\right], P_{i}=P_{m i}-E_{i}^{2} G_{i i}, C_{i j}=E_{i} E_{j} B_{i j}$, and $D_{i j}=E_{i} E_{j} G_{i j}$.

And for unit $i: \quad P_{m i}=$ mechanical power input;

$G_{i i}=$ driving point conductance;

$E_{i}=$ constant voltage behind the direct axis transient reactance;

$\omega_{i}, \delta_{i}=$ generator rotor speed and angle deviation, respectively;

$M_{i}=$ moment of inertia;

$B_{i j} G_{i j}=$ transfer susceptance, conductance in the reduced bus admittance matrix.

Define the center of angle

$$
\begin{gathered}
\delta_{0} \stackrel{\Delta}{=} 1 / M_{T} \sum_{i=1}^{n} M_{i} \delta_{i} \\
M_{T} \stackrel{\Delta}{=} \sum_{i=1}^{n} M_{i}
\end{gathered}
$$

The new angles and speeds relative to this reference

$$
\begin{gathered}
\theta_{i} \stackrel{\Delta}{=} \delta_{i}-\delta_{0} \\
\widetilde{\omega}_{i} \stackrel{\Delta}{=} \omega_{i}-\omega_{0}
\end{gathered}
$$

This transformation not only offers physical insight to the transient stability problem formulation in general, but in particular provides a concise framework for the analysis of system with transfer conductance. Expressed in these new definitions, the transient energy function can be written as

$$
\begin{aligned}
& V=1 / 2 \sum_{i=1}^{n} M_{i} \widetilde{\omega}_{i}^{2}-\sum_{i=1}^{n} P_{i}\left(\theta_{i}-\theta_{i}^{s}\right)-\sum_{i=1}^{n-1} \sum_{j=i+1}^{n}\left[C_{i j}\left(\cos \theta_{i j}-\cos \theta_{i j}^{s}\right)-\int_{\theta_{i}^{s}+\theta_{j}^{s}}^{\theta_{i}+\theta_{j}} D_{i j} \cos \theta_{i j} d\left(\theta_{i}+\theta_{j}\right)\right] \\
& =V_{k}+V_{p}
\end{aligned}
$$


where the kinetic energy is $V_{k}=1 / 2 \sum_{i=1}^{n} M_{i} \widetilde{\omega}_{i}^{2}$ and the potential energy is

$V_{p}=\sum_{i=1}^{n} P_{i}\left(\theta_{i}-\theta_{i}^{s}\right)-\sum_{i=1}^{n-1} \sum_{j=i+1}^{n}\left[C_{i j}\left(\cos \theta_{i j}-\cos \theta_{i j}^{s}\right)-\int_{\theta_{i}^{s}+\theta_{j}^{s}}^{\theta_{i}+\theta_{j}} D_{i j} \cos \theta_{i j} d\left(\theta_{i}+\theta_{j}\right)\right]$

The transient energy function lays down a foundation for Direct Methods and stability margin assessment.

\section{A.5.2 Potential Energy Boundary Surface (PEBS)}

An example of PEBS for a three-machine system is shown in Figure A. 14.

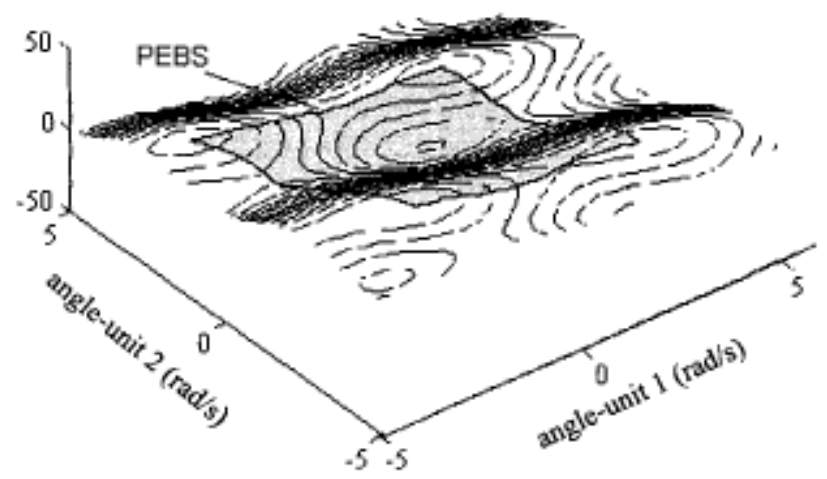

Figure A. 15. Equipotential Energy Surfaces for Three-Machine System

The synchronous equilibrium points are the sets of system variables which satisfy the equations of motions when the derivatives of speed and angle with respect to the center of angle are zero. The projection of the stable equilibrium point on the space of angles corresponds to a minimal of potential energy on the surface. The region of the potential energy surface in the neighborhood of the SEP is surrounded by a set of saddle points, unstable equilibrium point. The surface or hyper-surface that connects all the UEPs is termed the Potential Energy Boundary Surface (PEBS). The fault-on trajectory is followed until the projected trajectory $\delta(t)$ crosses the PEBS. The point in which the crossing occurs is called exit point, $\delta^{*}$. Then the critical energy is defined as the potential energy at $\delta^{*}, V_{C R}=V_{p}\left(\delta^{*}\right)$.

This method has the advantage of not requiring the calculation of unstable equilibrium points. The critical energy is defined as the first local maximum of the potential energy along the fault-on trajectory. The above terms are illustrated in Figure A. 16. 


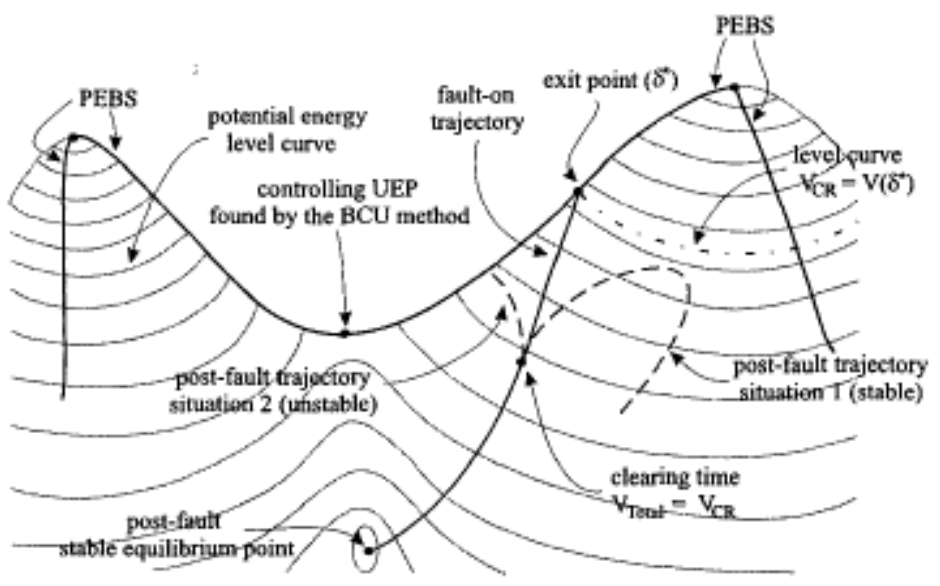

Figure A. 17. PEBS

\section{A.5.3 Boundary Controlling Unstable (BCU)}

Researchers tried to associate the unstable modes of the system with the location of unstable equilibrium points. Under the hypothesis about the vector field, the boundary of the attraction area is composed by the stable manifolds of the unstable equilibrium points. Figure A. 18 shows the three main computational tasks.

"Closest equilibrium point" calculates all unstable equilibrium point (UEP) around the stable equilibrium point and defines the VCR as the energy of the unstable equilibrium point, which has the lowest energy among them. For the first swing transient, the UEP closest to the trajectory of the disturbed system is the one that decides the transient stability of the system. It is considered the most efficient direct method for transient stability analysis.

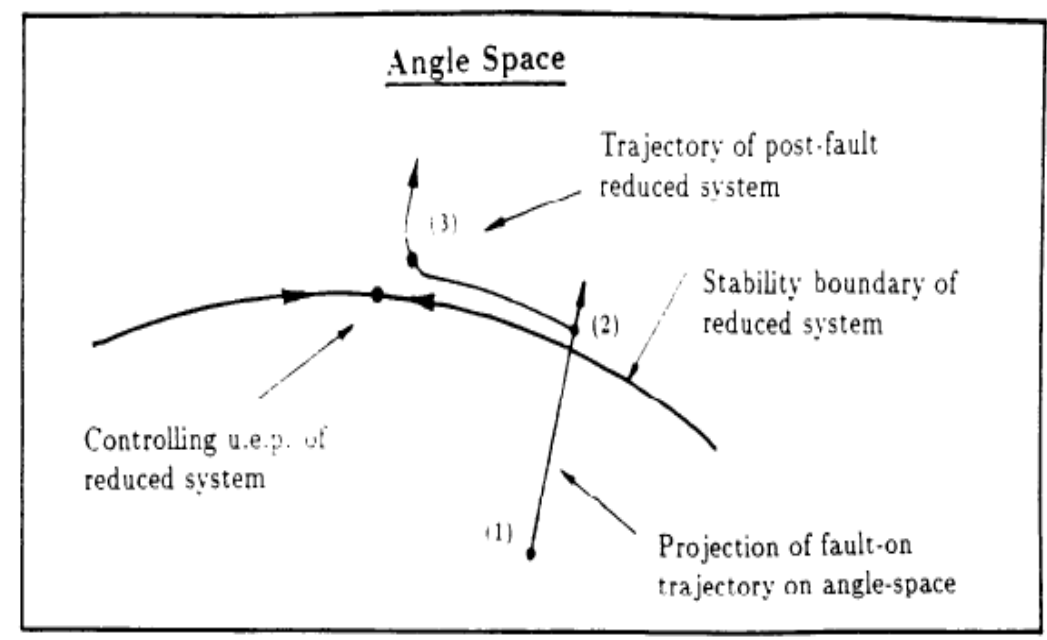

Figure A. 19. Three Main Computational Tasks in the BCU Method 
Suppose the reduced system is described by

$$
\dot{\delta}_{i}=\left(P_{i}-P_{e i}\right)-\frac{M_{i}}{M_{T}} P_{C O I}:=f_{i}(\delta) \quad i=1,2, \ldots, n
$$

\section{Algorithm:}

1. From the fault-on trajectory $(\delta(t), \widetilde{\omega}(t))$, detect the exit point $\delta^{*}$, which is the point the projected trajectory exits on the stability boundary of the reduced system

2. Use the point $\delta^{*}$ as the initial condition and integrate the post-fault reduced system (Equation (A.17)) to find the first local minimum of $\sum_{i=1}^{n}\left\|f_{i}(\delta)\right\|$, say at $\delta_{0}^{*}$.

3. Use the point $\delta_{0}^{*}$ as the initial guess to solve $\sum_{i=1}^{n}\left\|f_{i}(\delta)\right\|=0$, say at $\delta_{c 0}^{*}$.

4. Assign the controlling u.e.p. with respect to the fault-on trajectory to be $\left(\delta_{c 0}^{*}, 0\right)$.

\section{A.6 Hybrid Method}

The main idea is to combine the direct method with a simple method to make a first screening of contingencies. The most critical contingencies are then selected and in a second stage, the classic method is used to study these critical contingencies using a more complete model. Because the hybrid method is essentially the time domain simulation method, a stable case in time domain simulation remains stable in hybrid and unstable case remains unstable. The only difference is that hybrid method produces a stability index for fast derivation of stability limits.

\section{A.7 Artificial Learning}

The artificial learning (artificial neural networks, statistical pattern recognition (K-NN) Machine learning (Decision trees)) has been extended to transient stability assessment. The underlying principle is that, given a set of pre-classified examples along their characteristics attributes, derive a general rule that can explain the input-output relationship of pre-classified cases and correctly classify new or unseen cases. Specifically, the database would consist of numerous cases that have been assessed using time domain techniques. The attributes would consist of parameters such as generator outputs, critical line flows and relative phase angles. The output could be a stability margin or a simple classification (secure/insecure).

\section{A.8 Case Study}

\section{A.8.1 Transient Stability Boundary Visualization [6], [10], [11]}

In these studies, the security region is defined in controllable variables space, such as power injection space using hyperplanes. A hypothesis is introduced that reactive power is balanced locally and active 
power has little impact on the voltage profile. Through the linear transform, the dynamic security region in power injection space can be mapped to that in node angle space.

Assuming that the original system can be separated into two groups, the system considered was reduced to a simplified one-machine infinite bus (OMIB) system. The transient energy function was evaluated for transient stability analysis. This method only considers the constant impedance load and simplified generator model. An example of three-dimension security regions for the reduced equivalent system is shown in Figure A. 20.

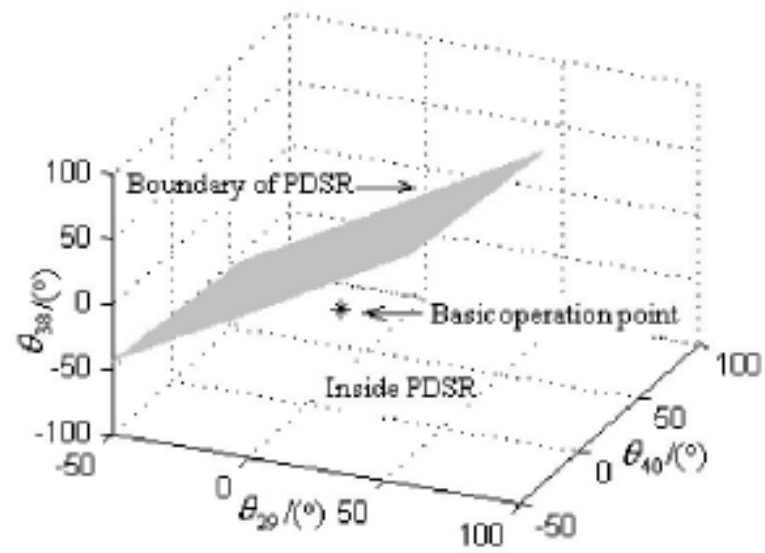

Figure A. 21. Three-dimensional Illustration of Security Region for Equivalent System [10]

\section{A.8.2 Transient Stability Analysis by the Method of Hyperplanes [18]}

In [18], each trajectory can be classified as asymptotically stable, maximal or unstable, as shown in Figure A. 22. The maximal trajectories are neither stable nor unstable. They form the boundary of the region of stability (or separatrix). A singular point is the equilibrium of the post-fault trajectories. A type 0 point is the stable operating point of the system; all others are unstable. The type 1 points are simple saddle points.

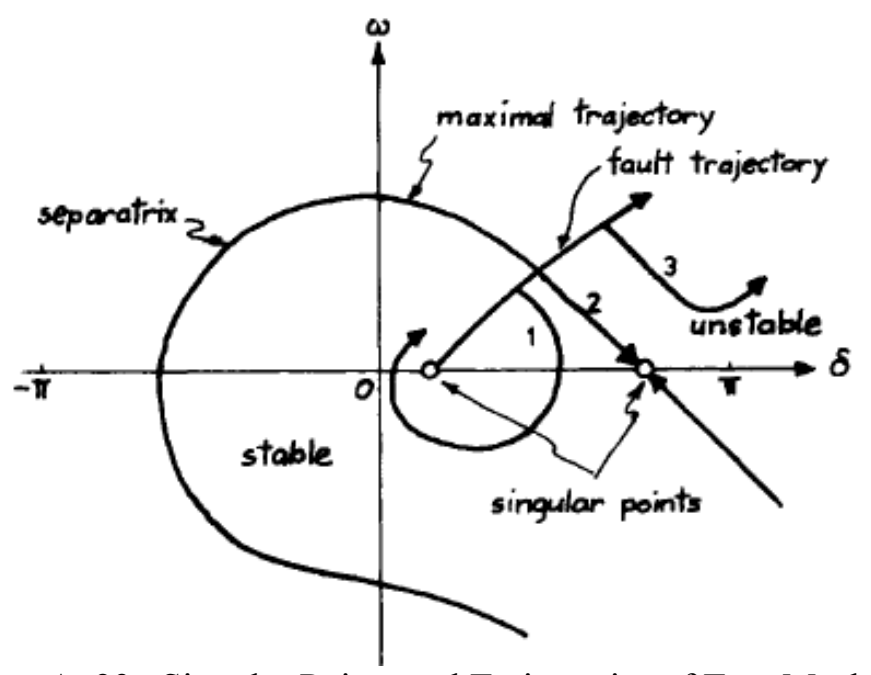

Figure A. 23. Singular Points and Trajectories of Two Machines 
The boundary of the region of stability in the state space (often called the separatrix) was then approximated by a number of hyperplanes, each of which is tangent to the separatrix. The equations of the hyperplanes were found by considering the dynamics of the system near each of a class of unstable singular points. However, the separatrix is a smooth surface at a type 1 point and it is not the case at a type 2 singular point, as illustrated in Figure A. 24. The approximation of separatrix with hyperplane at type 2 singular points yields significant estimation about the security region boundary. The experimental results show that it works for only about $60 \%$ of cases considered.

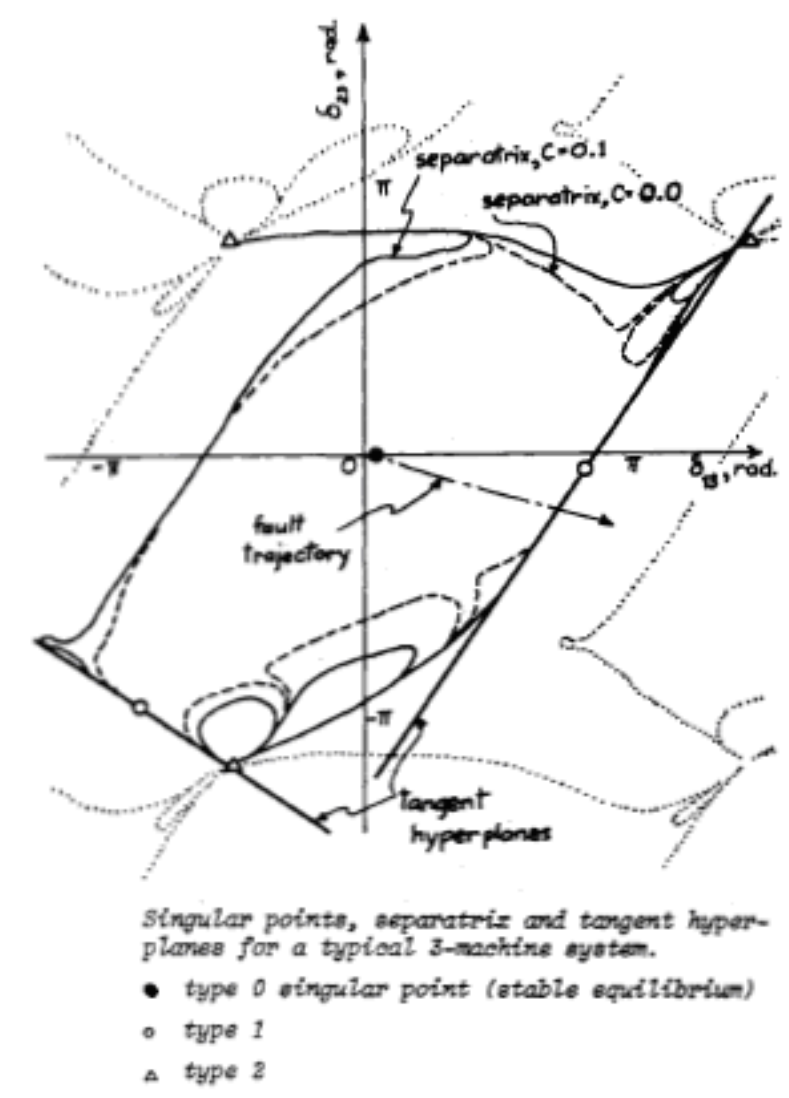

Figure A. 25. Singular Points, Separatrix, and Tangent Hyperplanes

\section{A.8.3 A Tool for on-line Stability Determination and Control [14]}

An approach to on-line dynamic security assessment using PMU measurements in conjunction with off-line transient stability analysis was developed. It automatically builds decision trees based on a preclassified set of examples. The rules then obtained relate the system stability to pre-fault static parameters of the system. A deductive inference method is then used to classify unseen cases in real time. The proposed scheme is tested on the Entergy online operational model, which consists of around 240 generators, 2000 buses, and 2100 lines. For each of the 56 operating conditions, there are a total 280 contingencies to evaluate the transient stability. This results in a total of $(280 * 56) 15680$ cases. Twenty percent of these cases are used as the test set (TS) and the rest of the cases are included in the learning set (LS). Each case is simulated in Powertech lab's TSAT, and two security criteria, transient stability and damping, are checked. 


\section{A.9 Stability Margin and Sensitivities Analysis [19], [20]}

The transient stability margin is given as

$$
\varepsilon=V_{C R}-V_{c l}
$$

where $V_{C R}$ and $V_{c l}$ denote the system critical energy and transient energy at fault clearing moment.

Direct methods open possibilities toward on-line analytic sensitivity analysis and preventative control. Numerical methods have been developed to use the sensitivities of the energy margin $\Delta \mathrm{V}$ to changes in system parameters to determine system stability limits. In [20], an approach of finding the sensitivity of $\Delta \mathrm{V}$ using a repetitive analysis through the transient energy function method and linearized margin assumption was used in the computation of interface low limits with power flow distribution factors. A theoretical foundation has been laid down in [19] to decide how to make changes in the parameters, through action such as generation rescheduling and load shedding, to improve the system's transient behavior. It was found that for small changes in generation, the first-order sensitivity technique quickly gave the new system's stability energy margin with a high degree of accuracy.

\section{A.10 Approximation Techniques for Security Regions}

\section{A.10.1 Hyperplane and Quadratic Approximations of the Security Region}

One of the important problems that power system analysts and operators mean when they use the concept of the power system security region is the problem of description of the security region's boundary. The simple tabular description is not adequate to the purposes of visualization and practical use by system operators and in the automated security assessment systems. There is a need for an analytical description and/or approximation of the boundary. The analytical description usually means the use of linear or nonlinear inequalities applied to a certain number of critical parameters such as power flows, load levels, voltage magnitudes, etc.; if all inequalities are satisfied, the analyzed operating point is considered to be inside the security region; if any of the inequalities is violated, the point is considered to be outside the security region. The approximation means a sort of interpolation between the boundary points obtained by any of the methods considered in this section. It can be used as a part of the analytical boundary description (for the automated security assessment systems), or separately for the purposes of visualization. The simplest approximation uses linear inequalities. The first known use of the approximation ideas was apparently related to the operating nomograms - see [4] for more details. The operating nomograms are usually represented visually as piecewise linear contours on a plane of two critical parameters. If three critical parameters are involved, the nomogram is represented by a number of contour lines; each of them corresponds to a certain value of the third parameter. It becomes difficult to visualize a nomogram for four or more critical parameters. The natural extension of the linearized stability nomograms for three or more critical parameters is based on the use of hyperplanes - the planes that are defined in the multidimensional parameter space as approximations of the stability boundary. These efforts are described in voltage stability boundary approximation [80], transient/dynamic stability boundary approximation [6], [17], [18], and other works. 
In Russia, in a number of emergency control algorithms, a nonlinear approximation was successfully used to provide an analytical description of the stability boundary [20]. These approaches employ quadratic inequalities. The inequalities are applied to the nodal power injections, cut-set power flows, and other parameters. The coefficients of these inequalities are pre-calculated offline based on multiple time domain or steady-state stability simulations.

The hyperplane and quadratic approximations have a number of significant advantages:

- They allow quick analysis of the stability margin in real time

- Because of their formal mathematical nature, they allow simultaneously consideration of thermal, voltage stability, transient stability and other constraints within the same framework.

\section{A.10.2 ANN-based Techniques [21], [4], [17], [22]-[30]}

The idea behind the techniques based on the artificial neural networks is to select a set of critical parameters such as power flows, loads, and generator limits, and then train an ANN on a set of simulation data to estimate the security margin. The ANN model de facto provides an approximation of the security boundary. The advantages of the ANN models include their ability to accommodate nonlinearities and their fast performance in real time. At the same time, there are difficulties associated with building the training datasets and ANN training.

\section{A.10.3 Pattern recognition methods}

Pattern recognition methods establish a relationship between some selected parameters and the location of the system operating point with respect to the security boundary. Initially, training sets of stable and unstable operating points are generated, and a space reduction process is applied to reduce the dimensionality of the system model. Then the classifier functions (decision rules) are determined using the training set. This function is engaged in real time to determine the stability margin of a given contingency [21], [31], [32].

\section{A.10.4 QuickStab algorithm}

QuickStab algorithm is an alternative method to quickly and approximately evaluate the voltage stability margin in a given loading direction. This technology was originally developed by Paul Dimo. It includes the voltage stability practical criterion $d Q / d V<0$ and Dimo's network nodal equivalents (so called Zero Power Balance Networks or REI equivalents). Dimo's finding is that under certain modeling assumptions the practical stability margin can be expressed as a straightforward formula applied to the nodal equivalents [33], [34].

\section{A.10.5 Delta-plane method}

Delta-plane method [35] is a new robust method for finding the power system load flow feasibility boundary on the plane defined by any three vectors of dependent variables (nodal voltages), called the Delta-plane. The method exploits some quadratic and linear properties of the load flow equations (X-ray theorem, [36]) and the power flow Jacobian written in rectangular coordinates. An advantage of the method is that it does not require an iterative solution of nonlinear equations (except the eigenvalue 
problem). Besides benefits of direct calculation of the power flow feasibility boundary points and visualization, the method is a useful tool for topological studies of power system multi-solution structures and stability regions. A disadvantage is that although the method works accurately in the state space, a mapping of its results into the parameter space is not a straightforward and accurate operation.

\section{A.11 Bibliography}

[1] Parashar M, Electric Power Group; A Agarwal, Electric Power Group; Y Makarov, Pacific Northwest National Laboratory; and I Dobson, University of Wisconsin, Madison. December 2007. "Real Time Voltage Security Assessment (RTVSA) Algorithms \& Framework." Prepared for California Independent System Operator by Consortium for Electric Reliability Technology Solutions, funded by California Public Interest Energy Research Transmission Research Program, Contract No. 500-02-004, MR-041. Available at http://certs.lbl.gov/certs-rt-pubs.html.

[2] Wu F and S Kumagai. 1982. "Steady-State Security Regions of Power Systems." IEEE Trans. on Circuits and Systems 29(11):703-711.

[3] Kaye R and F Wu. 1982. "Dynamic Security Regions of Power Systems." IEEE Trans. on Circuits and Systems 29(9):612-623.

[4] McCalley JD, S Wang, Q-L Zhao, G-Z Zhou, RT Treinen, and A D Papalexopoulos. 1997. "Security Boundary Visualization for Systems Operation." IEEE Trans. on Power Systems 12(2):940-947.

[5] Su J, Y Yu, H Jia, P Li, N He, Z Tang, and H Fu. 2002. "Visualization of Voltage Stability Region of Bulk Power System." Proceedings of International Conference on Power System Technology PowerCon 2002, Vol. 3, October 13-17, 2002, pp. 1665-1668.

[6] Zeng Y and Y Yu. 2002. "A Practical Direct Method for Determining Dynamic Security Regions of Electrical Power Systems." Proceedings of International Conference on Power System Technology PowerCon 2002, Vol. 2, October 13-17, 2002, pp. 1270-1274.

[7] Li P, Y Yu, H Jia, J Su, and J Zhao. 2002. "A Study on Boundary of Small Disturbance Stability Region." Proceedings of International Conference on Power System Technology PowerCon 2002, Vol. 2, October 13-17, 2002, pp. 1228-1232.

[8] Lee ST. 2003. "Community Activity Room as a New Tool for Transmission Operation and Planning Under a Competitive Power Market." Proceedings of 2003 IEEE Bologna Power Tech Conference, Vol. 4, June 23-26, 2003.

[9] Dolloff PA, D Adams, S Anderson, MY Vaiman, MM Vaiman, M Povolotskiy, and DT Bradshaw. 2008. "Evaluate Real-time System Reliability Using Boundary-based Concept for EKPC System." Proceedings of the 2008 PES General Meeting, Paper PESGM2008-001455, July 20-24, 2008.

[10] Zeng Y, Y Yu, and H Jia. 2005. "Theoretical Explanation of Hyper-Plane Boundary of Dynamic Security Region." Proceedings of Canadian Conference on Electrical and Computer Engineering, May 1-4, 2005, pp. 1946-1949.

[11] Dong C and Y Yu. 2005. "PDSR in Phase Angle Space and SPM Based Security Monitoring of Power Systems.” IEEE Region 10 TENCON 2005, November 2005, pp. 1-5.

[12] Rahimi FA, MG Lauby, JN Wrubel, and KL Lee. 1993. "Evaluation of the Transient Energy Function Method for On-line Dynamic Security Analysis." IEEE Trans. on Power Systems 8(2):497-507. 
[13] Alberto LDC, FHJR Silva, and NG Bretas. 2001. "Direct Methods for Transient Stability Analysis in Power Systems: State of Art and Future Perspectives." Proceedings of IEEE Porto Power Tech Proceedings, Vol. 2, September 10-13, 2001.

[14] Bettiol AL, Y Zhang, L Wehenkel, and M Pavella. 1997. "Transient Stability Investigations on a Brazilian Network by SIME." Proceedings of Fourth International Conference on Advances in Power System Control, Operation and Management APSCOM-97, Paper 450, Vol. 1, November 11-14, 1997, pp. L1-L6.

[15] Athay T, R Podmore, and S Virmani. 1979. "A Practical Method for the Direct Analysis of Transient Stability." IEEE Trans. on Power Apparatus and Systems, Vol. PAS-98, No. 2, March 1979, pp. 573-584.

[16] Chiang H-D, FF Wu, and PP Varaiya. 1994. "A BCU Method for Direct Analysis of Power System Transient Stability.” IEEE Trans. on Power Systems 9(3):1194-1208.

[17] Maria GA, C Tang, and J Kim. 1990. "Hybrid Transient Stability Analysis [Power Systems]." IEEE Trans. on Power Systems 5(2):384-393.

[18] Yee H and BD Spalding. 1977. "Transient Stability Analysis of Multimachine Power Systems by the Method of Hyperplanes." IEEE Trans. on Power Apparatus and Systems 96(1, Part 1):276-284.

[19] Tong J, H-D Chiang, and TP Conneen. 1993. "A Sensitivity-based BCU Method for Fast Derivation of Stability Limits in Electric Power Systems.” IEEE Trans. on Power Systems 8(4):1418-1428.

[20] El-Kady MA, CK Tang, VF Carvalho, AA Fouad, and V Vittal. 1986. "Dynamic Security Assessment Utilizing the Transient Energy Function Method." IEEE Trans. on Power Systems 1(3):284-291.

[21] Chiang H-D, F Wu, and P Varaiya. 1987. "Foundations of Direct Methods for Power System Transient Stability Analysis.” IEEE Trans. on Circuits and Systems 34(2):160-173.

[22] Hiskens IA and RJ Davy. 2001. "Exploring the Power Flow Solution Space Boundary." IEEE Power Engineering Review 21(8):57-57.

[23] Dobson I. 1992. "Observations on the Geometry of Saddle Node Bifurcation and Voltage Collapse in Electrical Power Systems." IEEE Trans. on Circuits and Systems I: Fundamental Theory and Applications 39(3):240-243.

[24] Vittal V, G Heydt, and AP Sakis Meliopoulos. 2008. “A Tool for On-Line Stability Determination and Control for Coordinated Operations Between Regional Entities Using PMUs.” PSERC Project Report S-27, PSERC Publication 08-01. Available online: http://www.pserc.wisc.edu/ecow/get/publicatio/reports/2008report/.

[25] Begovic MM and AG Phadke. 1992. "Control of Voltage Stability Using Sensitivity Analysis." IEEE Trans. on Power Systems 7(1):114-123.

[26] Dobson I and L Lu. 1993. "New Methods for Computing a Closest Saddle Node Bifurcation and Worst Case Load Power Margin for Voltage Collapse." IEEE Trans. on Power Systems 8(3):905-913.

[27] Djukanovic M, DJ Sobajic, and Y-H Pao. 1993. "Learning Tangent Hypersurfaces for Fast Assessment of Transient Stability." Proceedings of the Second International Forum on Applications of Neural Networks to Power Systems ANNPS '93, April 19-22, 1993, pp. 124-129.

[28] Yu Y, Y Zeng, C Huang, ST Lee, and P Zhang. 2004. “A Practical Direct Method for Determining Dynamic Security Regions of Electrical Power Systems by Power Perturbation Analysis." 
Proceedings of International Conference on Electrical Engineering ICEE2004, July 4-8, 2004, Sapporo, Japan.

[29] Dolloff PA, D Adams, S Anderson, MY Vaiman, MM Vaiman, M Povolotskiy, and DT Bradshaw. 2008. "Evaluate Real-time System Reliability Using Boundary-based Concept for EKPC System." Proceedings of the 2008 PES General Meeting, Paper PESGM2008-001455, July 20-24, 2008.

[30] Sovalov SA and VA Semenov. 1988. "Emergency Control in Power Systems." Energoatomizdat, Moscow (in Russian).

[31] Sauer PW, K Tomsovic, J Dagle, S Widergren, T Nguyen, and L Schienbein. 2004. "Integrated Security Analysis.” Final Report, CERTS, July 2004. Available online: http://certs.lbl.gov/CERTS_P_RealTime.html.

[32] El-Sharkawi MA, RJ Marks, ME Aggoune, DC Park, MJ Damborg, and LE Atlas. 1989. "Dynamic Security Assessment of Power Systems Using Back Error Propagation Artificial Neuron Networks." Proceedings of the 2nd Symposium on Expert System Applications to Power Systems, Seattle, Washington, July 1989.

[33] Kumar R, A Ipahchi, V Brandwajan, MA El-Sharkawi, and G Cauley. 1991. "Neuron Networks for Dynamic Security Assessment of Large Scale Power Systems: Requirements Overview." Proceedings of 1st International Forum on Applications of Neuron Networks to Power Systems, Seattle, Washington, July 1991, pp. 65-71.

[34] EI-Keib AA and X Ma. 1995. "Application of Artificial Neural Networks in Voltage Stability Assessment." IEEE Trans. on Power System 10(4):1890-1896.

[35] Chauhan S and MP Dava. 1997. "Kohonen Neural Network Classifier for Voltage Collapse Margin Estimation." Electric Machines and Power Systems 25(6):607-619.

[36] Mansour Y, AY Chang, J Tamby, E Vaahedi, BR Corns, and MA El-Sharkawi. 1997. "Large Scale Dynamic Security Screening and Ranking Using Neuron Networks." IEEE Trans. on Power Systems 12(2):954-960.

[37] Wan HB and YH Song. 1998. "Hybrid Supervised and Unsupervised Neural Network Approach to Voltage Stability Analysis." Electric Power System Research 47(2):115-122.

[38] Dinavahi VR and SC Srivastava. 2001. “ANN Based Voltage Stability Margin Prediction." IEEE Power Engineering Society Summer Meeting 2001 Vol. 2, July 15-19, 2001, pp. 1275-1280.

[39] Sittithumwat A and K Tomsovic. 2002. "Dynamic Security Margin Estimation Using Artificial Neural Networks." IEEE Power Engineering Society Summer Meeting 2002, Vol. 3, pp. $1322-1327$.

[40] Salamaa MM, EM Saieda, MM Abou-Elsaada, and EF Gharianyb. 2001. "Estimating the Voltage Collapse Proximity Indicator Using Artificial Neural Network." Energy Conversion and Management 42(1):69-79.

[41] Hakim H. 1992. "Application of Pattern Recognition in Transient Security Assessment." Journal of Electrical Machines and Power Systems, 20:1-15.

[42] Wehenkel L. 1998. Automatic Learning Techniques in Power Systems. Kluwer Academic Publishers, Massachusetts.

[43] Aldea C and SC Savulescu. 2004. "Evaluation of the Stability Reserve of Transelectrica's Transmission System by Using Quickstab Professional." WEC Regional Energy Forum - FOREN 2004, Neptun, June 13-17, 2004.

[44] Dimo P. 1971. “L'Analyse Nodale des Réseaux D’Énergie.” Eyrolles. 
[45] Makarov YV, DJ Hill, and Z-Y Dong. 2000. "Computation of Bifurcation Boundaries for Power Systems: A New $\Delta$-Plane Method." IEEE Trans. on Circuits and Systems I: Fundamental Theory and Applications 47(4):536-544.

[46] Makarov YV, DJ Hill, and IA Hiskens. 2000. "Properties of Quadratic Equations and Their Application to Power System Analysis." Electrical Power and Energy Systems 22:313-323.

[47] Parashar M, Electric Power Group; A Agarwal, Electric Power Group; Y Makarov, Pacific Northwest National Laboratory; and I Dobson, University of Wisconsin, Madison. 2008. "RealTime Voltage Security Assessment (RTVSA): Summary Report.” Prepared for California Independent System Operator by Consortium for Electric Reliability Technology Solutions, funded by California Public Interest Energy Research Transmission Research Program, Contract No. 500-02-004, MR-041, January 21, 2008. Available at: http://certs.lbl.gov/pdf/task3-rtvsasummary-rpt.pdf 



\section{Appendix B}

\section{Hyperplanes}





\section{Appendix B}

\section{Hyperplanes}

The hyperplanes' coefficients which have been found in the descriptor parameter space are shown in Table B-1.

Table B- 1. Coefficients of hyper-planes

\begin{tabular}{|c|c|c|c|c|c|c|c|c|}
\hline & $\begin{array}{l}d_{1} \\
\left(n_{1}\right)\end{array}$ & $\begin{array}{l}d_{2} \\
\left(n_{2}\right)\end{array}$ & $\begin{array}{l}d_{3} \\
\left(n_{3}\right)\end{array}$ & $\begin{array}{l}d_{4} \\
\left(n_{4}\right)\end{array}$ & $\begin{array}{l}d_{5} \\
\left(n_{5}\right)\end{array}$ & $\begin{array}{l}d_{6} \\
\left(n_{6}\right)\end{array}$ & $\begin{array}{l}d_{7} \\
\left(n_{7}\right)\end{array}$ & $\begin{array}{l}d_{8} \\
\left(n_{8}\right)\end{array}$ \\
\hline 1 & -0.0681 & 0.1449 & 0.0622 & -0.0554 & -0.559 & 0.6724 & 0.4495 & -0.0271 \\
\hline 2 & -0.0276 & 0.1425 & 0.0667 & -0.0111 & -0.5343 & 0.6077 & 0.5619 & 0.0612 \\
\hline 3 & -0.0278 & 0.1425 & 0.0670 & -0.0108 & -0.5341 & 0.6079 & 0.5621 & 0.0601 \\
\hline 4 & -0.0338 & 0.1431 & 0.0663 & -0.0122 & -0.5396 & 0.6198 & 0.5443 & 0.0474 \\
\hline 5 & -0.0294 & 0.1633 & 0.0745 & -0.0060 & -0.5468 & 0.6675 & 0.4694 & -0.0438 \\
\hline 6 & -0.0982 & 0.1440 & 0.0574 & -0.0885 & -0.5671 & 0.7091 & 0.3536 & -0.0949 \\
\hline 7 & 0.1606 & -0.1173 & -0.0474 & 0.1154 & 0.5674 & -0.7575 & -0.1704 & 0.1417 \\
\hline 8 & -0.0007 & -0.1611 & -0.0602 & 0.0475 & 0.5432 & -0.6194 & -0.5367 & 0.0376 \\
\hline 9 & -0.1107 & 0.1509 & 0.0525 & -0.0380 & -0.5674 & 0.7328 & 0.2613 & -0.183 \\
\hline 10 & -0.1540 & 0.1280 & 0.2243 & 0.1626 & -0.2055 & 0.4879 & 0.4923 & -0.6004 \\
\hline 11 & -0.1608 & 0.1299 & 0.0459 & -0.0329 & -0.5512 & 0.7613 & 0.0604 & -0.2589 \\
\hline 12 & -0.0306 & 0.2016 & 0.0870 & 0.0103 & -0.5025 & 0.7475 & 0.1209 & -0.3533 \\
\hline 13 & -0.0289 & 0.1938 & 0.0832 & -0.0016 & -0.5089 & 0.7125 & 0.3003 & -0.3129 \\
\hline 14 & -0.1940 & 0.1173 & 0.0295 & -0.1993 & -0.508 & 0.7333 & -0.0611 & -0.3289 \\
\hline 15 & 0.1521 & -0.1205 & -0.0505 & 0.1068 & 0.5687 & -0.7524 & -0.2044 & 0.1306 \\
\hline 16 & -0.1050 & 0.0858 & 0.0855 & 0.0911 & -0.4739 & 0.5425 & 0.5982 & 0.2989 \\
\hline 17 & -0.1601 & 0.1380 & 0.0251 & -0.0646 & -0.5337 & 0.7444 & -0.0284 & -0.3329 \\
\hline 18 & -0.1364 & 0.1378 & 0.2041 & 0.1358 & -0.2838 & 0.5387 & 0.533 & -0.4975 \\
\hline 19 & -0.1630 & 0.1269 & 0.0370 & -0.0410 & -0.5548 & 0.7631 & 0.0222 & -0.2524 \\
\hline 20 & 0.0137 & -0.0189 & -0.0180 & 0.0276 & 0.3376 & -0.1994 & -0.7900 & -0.4695 \\
\hline 21 & 0.0164 & -0.0135 & -0.0190 & 0.0182 & 0.357 & -0.219 & -0.7128 & -0.5615 \\
\hline 22 & 0.1493 & 0.0837 & 0.0637 & 0.2070 & -0.2529 & 0.1395 & 0.8241 & 0.4015 \\
\hline 23 & 0.2652 & 0.0394 & 0.0349 & 0.1887 & 0.0909 & -0.3305 & 0.7701 & 0.4251 \\
\hline 24 & -0.2075 & 0.0937 & 0.0719 & 0.0661 & -0.5603 & 0.738 & 0.2734 & 0.0732 \\
\hline 25 & 0.1258 & -0.0965 & -0.0747 & -0.0307 & 0.5786 & -0.7339 & -0.302 & -0.056 \\
\hline 26 & 0.1232 & 0.0950 & 0.0600 & 0.0164 & -0.2895 & 0.189 & 0.846 & 0.3692 \\
\hline 27 & 0.1478 & 0.0788 & 0.0638 & 0.0311 & -0.2015 & 0.0741 & 0.874 & 0.3941 \\
\hline 28 & 0.0417 & -0.085 & -0.0424 & 0.0260 & 0.486 & -0.4647 & -0.6845 & -0.2602 \\
\hline 29 & 0.0486 & -0.092 & -0.046 & 0.0211 & 0.5163 & -0.5152 & -0.6184 & -0.2684 \\
\hline 30 & 0.0043 & 0.1665 & 0.0794 & 0.0288 & -0.5194 & 0.6174 & 0.5600 & -0.0231 \\
\hline 31 & -0.0898 & 0.1609 & 0.0683 & -0.0162 & -0.3893 & 0.6952 & -0.1356 & -0.5548 \\
\hline 32 & -0.1214 & 0.1671 & 0.0748 & -0.0310 & -0.4237 & 0.721 & -0.0644 & -0.4972 \\
\hline 33 & -0.0625 & 0.1816 & 0.0752 & -0.0560 & -0.5143 & 0.7285 & 0.2317 & -0.3247 \\
\hline 34 & -0.0654 & 0.1820 & 0.0735 & -0.0439 & -0.5116 & 0.7298 & 0.2089 & -0.3424 \\
\hline 35 & -0.0631 & 0.1824 & 0.0728 & -0.0433 & -0.5143 & 0.7299 & 0.2154 & -0.3346 \\
\hline 36 & -0.0572 & 0.1821 & 0.0746 & -0.0560 & -0.5215 & 0.7222 & 0.2741 & -0.2937 \\
\hline 37 & -0.0763 & 0.1019 & 0.0394 & -0.0138 & -0.2192 & 0.5045 & -0.7572 & -0.3257 \\
\hline
\end{tabular}




\begin{tabular}{|c|c|c|c|c|c|c|c|c|}
\hline & $\begin{array}{l}d_{1} \\
\left(n_{1}\right)\end{array}$ & $\begin{array}{l}d_{2} \\
\left(n_{2}\right)\end{array}$ & $\begin{array}{l}d_{3} \\
\left(n_{3}\right)\end{array}$ & $\begin{array}{l}d_{4} \\
\left(n_{4}\right)\end{array}$ & $\begin{array}{l}d_{5} \\
\left(n_{5}\right)\end{array}$ & $\begin{array}{l}d_{6} \\
\left(n_{6}\right)\end{array}$ & $\begin{array}{l}d_{7} \\
\left(n_{7}\right)\end{array}$ & $\begin{array}{l}d_{8} \\
\left(n_{8}\right)\end{array}$ \\
\hline 38 & -0.0898 & 0.1609 & 0.0683 & -0.0162 & -0.3893 & 0.6952 & -0.1356 & -0.5548 \\
\hline 39 & 0.0423 & 0.1318 & 0.0663 & 0.0411 & -0.4577 & 0.4478 & 0.7237 & 0.2023 \\
\hline 40 & -0.1085 & 0.1648 & 0.0722 & -0.0249 & -0.4100 & 0.7114 & -0.094 & -0.5218 \\
\hline 41 & -0.0743 & 0.1784 & 0.0745 & -0.0425 & -0.4803 & 0.7342 & 0.1000 & -0.4189 \\
\hline 42 & -0.1214 & 0.1671 & 0.0748 & -0.0310 & -0.4237 & 0.721 & -0.0644 & -0.4972 \\
\hline 43 & -0.0737 & 0.1793 & 0.0734 & -0.0367 & -0.486 & 0.7337 & 0.1133 & -0.4104 \\
\hline 44 & -0.0718 & 0.1799 & 0.0730 & -0.0365 & -0.4893 & 0.7344 & 0.1211 & -0.4029 \\
\hline 45 & -0.0707 & 0.1800 & 0.0746 & -0.0433 & -0.4897 & 0.7346 & 0.1343 & -0.3971 \\
\hline 46 & -0.0867 & 0.1322 & 0.0536 & -0.0157 & -0.3018 & 0.6141 & -0.5576 & -0.439 \\
\hline 47 & -0.1085 & 0.1648 & 0.0722 & -0.0249 & -0.41 & 0.7114 & -0.094 & -0.5218 \\
\hline 48 & -0.0402 & 0.1802 & 0.0842 & 0.0098 & -0.5274 & 0.7045 & 0.3827 & -0.1942 \\
\hline 49 & -0.0974 & -0.1213 & -0.0380 & 0.0819 & 0.4511 & -0.3758 & -0.7593 & -0.2155 \\
\hline 50 & -0.0055 & -0.1749 & -0.0624 & 0.0524 & 0.5431 & -0.6447 & -0.4863 & 0.1255 \\
\hline 51 & -0.0043 & -0.1760 & -0.0620 & 0.0506 & 0.5449 & -0.6505 & -0.4758 & 0.1276 \\
\hline 52 & -0.0831 & -0.1255 & -0.0400 & 0.0749 & 0.4573 & -0.3941 & -0.7514 & -0.2027 \\
\hline 53 & -0.0374 & 0.0559 & 0.0335 & -0.0173 & -0.195 & 0.1529 & 0.9562 & -0.1355 \\
\hline 54 & -0.0625 & 0.1816 & 0.0752 & -0.0560 & -0.5143 & 0.7285 & 0.2317 & -0.3247 \\
\hline 55 & -0.0743 & 0.1784 & 0.0745 & -0.0425 & -0.4803 & 0.7342 & 0.100 & -0.4189 \\
\hline 56 & -0.0974 & -0.1213 & -0.0380 & 0.0819 & 0.4511 & -0.3758 & -0.7593 & -0.2155 \\
\hline 57 & -0.0214 & 0.1769 & 0.0799 & -0.0032 & -0.5316 & 0.6772 & 0.4483 & -0.1404 \\
\hline 58 & -0.0715 & 0.1821 & 0.0694 & -0.0170 & -0.504 & 0.7300 & 0.158 & -0.3804 \\
\hline 59 & -0.0641 & 0.1836 & 0.0681 & -0.0186 & -0.5137 & 0.7313 & 0.1834 & -0.3534 \\
\hline 60 & 0.0192 & 0.1428 & 0.0486 & -0.0431 & -0.4802 & 0.4709 & 0.7085 & 0.1438 \\
\hline 61 & 0.0099 & 0.0816 & 0.0363 & -0.0413 & -0.2946 & 0.239 & 0.9199 & -0.0137 \\
\hline 62 & -0.0654 & 0.1820 & 0.0735 & -0.0439 & -0.5116 & 0.7298 & 0.2089 & -0.3424 \\
\hline 63 & -0.0737 & 0.1793 & 0.0734 & -0.0367 & -0.486 & 0.7337 & 0.1133 & -0.4104 \\
\hline 64 & -0.0055 & -0.1749 & -0.0624 & 0.0524 & 0.5431 & -0.6447 & -0.4863 & 0.1255 \\
\hline 65 & -0.0715 & 0.1821 & 0.0694 & -0.0170 & -0.504 & 0.7300 & 0.158 & -0.3804 \\
\hline 66 & -0.0292 & 0.1794 & 0.0791 & -0.0053 & -0.5325 & 0.6917 & 0.4088 & -0.1779 \\
\hline 67 & -0.0096 & 0.1862 & 0.0563 & -0.0292 & -0.5592 & 0.7085 & 0.3523 & -0.1496 \\
\hline 68 & -0.0848 & 0.1756 & 0.0681 & -0.0101 & -0.468 & 0.7252 & 0.0291 & -0.4599 \\
\hline 69 & -0.0168 & 0.1267 & 0.0524 & -0.0383 & -0.4046 & 0.4386 & 0.7768 & -0.1415 \\
\hline 70 & -0.0631 & 0.1824 & 0.0728 & -0.0433 & -0.5143 & 0.7299 & 0.2154 & -0.3346 \\
\hline 71 & -0.0718 & 0.1799 & 0.0730 & -0.0365 & -0.4893 & 0.7344 & 0.1211 & -0.4029 \\
\hline 72 & -0.0043 & -0.1760 & -0.0620 & 0.0506 & 0.5449 & -0.6505 & -0.4758 & 0.1276 \\
\hline 73 & -0.0641 & 0.1836 & 0.0681 & -0.0186 & -0.5137 & 0.7313 & 0.1834 & -0.3534 \\
\hline 74 & -0.0096 & 0.1862 & 0.0563 & -0.0292 & -0.5592 & 0.7085 & 0.3523 & -0.1496 \\
\hline 75 & -0.0288 & 0.1795 & 0.0786 & -0.0058 & -0.5331 & 0.6921 & 0.4076 & -0.1773 \\
\hline 76 & -0.0753 & 0.1794 & 0.0674 & -0.0130 & -0.487 & 0.7324 & 0.0751 & -0.4221 \\
\hline 77 & -0.0166 & 0.1303 & 0.0529 & -0.0381 & -0.4145 & 0.4541 & 0.7616 & -0.1428 \\
\hline 78 & -0.0572 & 0.1821 & 0.0746 & -0.0560 & -0.5215 & 0.7222 & 0.2741 & -0.2937 \\
\hline 79 & -0.0707 & 0.1800 & 0.0746 & -0.0433 & -0.4897 & 0.7346 & 0.1343 & -0.3971 \\
\hline 80 & -0.0831 & -0.1255 & -0.0400 & 0.0749 & 0.4573 & -0.3941 & -0.7514 & -0.2027 \\
\hline 81 & 0.0192 & 0.1428 & 0.0486 & -0.0431 & -0.4802 & 0.4709 & 0.7085 & 0.1438 \\
\hline
\end{tabular}




\begin{tabular}{lllllllll}
\hline & $\begin{array}{l}d_{1} \\
\left(n_{1}\right)\end{array}$ & $\begin{array}{l}d_{2} \\
\left(n_{2}\right)\end{array}$ & $\begin{array}{l}d_{3} \\
\left(n_{3}\right)\end{array}$ & $\begin{array}{l}d_{4} \\
\left(n_{4}\right)\end{array}$ & $\begin{array}{l}d_{5} \\
\left(n_{5}\right)\end{array}$ & $\begin{array}{l}d_{6} \\
\left(n_{6}\right)\end{array}$ & $\begin{array}{l}d_{7} \\
\left(n_{7}\right)\end{array}$ & $\begin{array}{l}d_{8} \\
\left(n_{8}\right)\end{array}$ \\
\hline 82 & -0.0848 & 0.1756 & 0.0681 & -0.0101 & -0.468 & 0.7252 & 0.0291 & -0.4599 \\
83 & -0.0753 & 0.1794 & 0.0674 & -0.0130 & -0.487 & 0.7324 & 0.0751 & -0.4221 \\
84 & -0.0202 & 0.1763 & 0.0792 & -0.0045 & -0.5316 & 0.6727 & 0.4579 & -0.1318 \\
85 & 0.0107 & 0.0867 & 0.0375 & -0.0417 & -0.3104 & 0.2579 & 0.9091 & -0.0018 \\
86 & -0.0689 & 0.0458 & -0.4794 & 0.6957 & 0.3100 & 0.1055 & -0.3929 & -0.1331 \\
87 & -0.7727 & 0.0033 & 0.0554 & -0.1896 & 0.1465 & 0.0636 & -0.5650 & -0.1379 \\
88 & -0.1727 & 0.3576 & -0.0425 & -0.1345 & 0.4673 & -0.6851 & 0.1392 & 0.3396 \\
89 & -0.0489 & -0.1387 & -0.0732 & 0.0495 & 0.5303 & -0.6365 & -0.4927 & 0.2035 \\
90 & -0.0143 & -0.1089 & -0.0939 & 0.0691 & 0.3002 & -0.5418 & 0.6447 & 0.4184 \\
91 & 0.0000 & 0.0000 & -0.3100 & 0.0000 & 0.9500 & 0.0000 & 0.0000 & 0.0000 \\
92 & 0.0000 & 0.0000 & -0.6000 & 0.8000 & 0.0000 & 0.0000 & 0.0000 & 0.0000
\end{tabular}

\begin{tabular}{|c|c|c|c|c|c|}
\hline & $\mathrm{L}$ & 34 & -1359 & 68 & -1429 \\
\hline 1 & -818 & 35 & -1352 & 69 & -647 \\
\hline 2 & -421 & 36 & -1316 & 70 & -1352 \\
\hline 3 & -420 & 37 & -1174 & 71 & -1415 \\
\hline 4 & -461 & 38 & -1484 & 72 & 1201 \\
\hline 5 & -637 & 39 & 135 & 73 & -1326 \\
\hline 6 & -1108 & 40 & -1401 & 74 & -1143 \\
\hline 7 & 1265 & 41 & -1443 & 75 & -882 \\
\hline 8 & 972 & 42 & -1421 & 76 & -1407 \\
\hline 9 & -1127 & 43 & -1415 & 77 & -675 \\
\hline 10 & 602 & 44 & -1417 & 78 & -1316 \\
\hline 11 & 1229 & 45 & -1376 & 79 & -1417 \\
\hline 12 & 1191 & 46 & -1339 & 80 & 672 \\
\hline 13 & 1111 & 47 & -1401 & 81 & -424 \\
\hline 14 & -2022 & 48 & -769 & 82 & -1429 \\
\hline 15 & 1202 & 49 & 726 & 83 & -1407 \\
\hline 16 & 1087 & 50 & 1205 & 84 & -776 \\
\hline 17 & -1626 & 51 & 1201 & 85 & -251 \\
\hline 18 & 425 & 52 & 672 & 86 & 5047 \\
\hline 19 & -1310 & 53 & 39 & 87 & 1713 \\
\hline 20 & -584 & 54 & -1364 & 88 & 2481 \\
\hline 21 & -736 & 55 & -1443 & 89 & 1539 \\
\hline 22 & 1475 & 56 & 726 & 90 & 2229 \\
\hline 23 & 1685 & 57 & -785 & 91 & 7306 \\
\hline 24 & 356 & 58 & -1344 & 92 & 3471 \\
\hline 25 & 176 & 59 & -1326 & & \\
\hline 26 & 600 & 60 & -424 & & \\
\hline 27 & 872 & 61 & -236 & & \\
\hline 28 & 13 & 62 & -1359 & & \\
\hline 29 & 39 & 63 & -1421 & & \\
\hline 30 & -402 & 64 & 1205 & & \\
\hline 31 & -1484 & 65 & -1344 & & \\
\hline 32 & -1339 & 6 & -877 & & \\
\hline 33 & -1364 & 67 & -1143 & & \\
\hline
\end{tabular}





\section{Appendix C}

Evaluate Different Transformation of Hyperplanes in Angle Space 



\section{Appendix C}

\section{Evaluate Different Transformations of Hyperplanes in Angle Space}

The hyperplanes in the power transfer space approximating security conditions are calculated off-line, and can be described in n-dimension space as

$$
\left\{\begin{array}{l}
n_{p, 11} P_{1}+\ldots+n_{p, 1 n} P_{n} \leq C_{P, 1} \\
n_{p, 21} P_{1}+\ldots+n_{p, 2 n} P_{n} \leq C_{P, 2} \\
\ldots \\
n_{p, s 1} P_{1}+\ldots+n_{p, s n} P_{n} \leq C_{P, s}
\end{array}\right.
$$

or in matrix form

$$
N^{t} P \leq L_{d}
$$

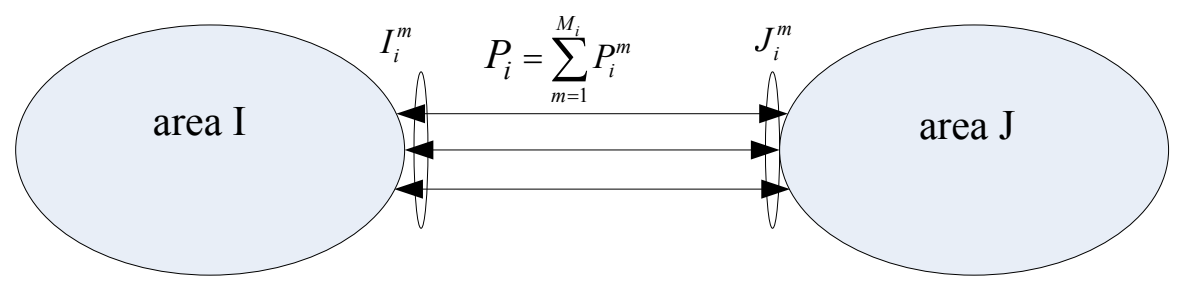

Figure C. 1. Power Flow on the Interface between Neighboring areas I and J

The interchange power between two neighboring areas, $P_{i}$, is the sum of all the power flows on the lines connecting them, which can be denoted by

$$
P_{i}=\sum_{m=1}^{M_{i}} P_{i}^{m}
$$

( $M_{i}$ is the number of power lines connecting two areas I and $\mathrm{J}$, and $P_{i}^{m}$ is the active power flow on the line between the bus $I_{i}^{m}$ and $J_{i}^{m}$ ).

The approximate relationship between the power flow and the voltage phasor angle is

$$
P_{i}^{m} \approx V\left(I_{i}^{m}\right) V\left(J_{i}^{m}\right) \frac{\sin \left(\delta\left(I_{i}^{m}\right)-\delta\left(J_{i}^{m}\right)\right)}{X\left(I_{i}^{m}, J_{i}^{m}\right)}
$$


It can be further approximated as a linear function of power angle difference and line reactance ${ }^{1}$, assuming the voltage magnitude is close to the unit value and the voltage angle difference is small:

$$
\begin{gathered}
P_{i}^{m} \approx V\left(I_{i}^{m}\right) V\left(J_{i}^{m}\right) \frac{\delta\left(I_{i}^{m}\right)-\delta\left(J_{i}^{m}\right)}{X\left(I_{i}^{m}, J_{i}^{m}\right)} \\
P_{i}^{m} \approx \frac{\delta\left(I_{i}^{m}\right)-\delta\left(J_{i}^{m}\right)}{X\left(I_{i}^{m}, J_{i}^{m}\right)}
\end{gathered}
$$

where $I_{i}^{m}, J_{i}^{m}$ are the bus index of the line, $\delta\left(I_{i}^{m}\right)$ and $\delta\left(J_{i}^{m}\right)$ are the voltage angles at bus $I_{i}^{m}$ and $J_{i}^{m}$ and $V\left(I_{i}^{m}\right)$ and $V\left(J_{i}^{m}\right)$ are the voltage magnitude at bus $I_{i}^{m}$ and $J_{i}^{m}$.

Inserting (C.5) into (C.3), we have

$$
\begin{aligned}
& P_{i}=\sum_{m=1}^{M_{i}} P_{i}^{m}=\sum_{m=1}^{M_{i}} V\left(I_{i}^{m}\right) V\left(J_{i}^{m}\right) \frac{\delta\left(I_{i}^{m}\right)-\delta\left(J_{i}^{m}\right)}{X\left(I_{i}^{m}, J_{i}^{m}\right)} \\
& =\left[\begin{array}{llllll}
\frac{V\left(I_{i}^{1}\right) V\left(J_{i}^{1}\right)}{X\left(I_{i}^{1}, J_{i}^{1}\right)} & \cdots & \frac{V\left(I_{i}^{M}\right) V\left(J_{i}^{M}\right)}{X\left(I_{i}^{M}, J_{i}^{M}\right)} & -\frac{V\left(I_{i}^{1}\right) V\left(J_{i}^{1}\right)}{X\left(I_{i}^{1}, J_{i}^{1}\right)} & \cdots & -\frac{V\left(I_{i}^{M}\right) V\left(J_{i}^{M}\right)}{X\left(I_{i}^{M}, J_{i}^{M}\right)}
\end{array}\right]\left[\begin{array}{l}
\delta\left(I_{i}^{1}\right) \\
\vdots \\
\delta\left(I_{i}^{M_{i}}\right) \\
\delta\left(J_{i}^{1}\right) \\
\vdots \\
\delta\left(J_{i}^{M_{i}}\right)
\end{array}\right]=g_{i}^{1} \theta_{i}
\end{aligned}
$$

Inserting (C.6) into (C.3), we have

$$
\begin{gathered}
P_{i}=\sum_{m=1}^{M_{i}} P_{i}^{m}=\sum_{m=1}^{M_{i}} \frac{\delta\left(I_{i}^{m}\right)-\delta\left(J_{i}^{m}\right)}{X\left(I_{i}^{m}, J_{i}^{m}\right)} \\
=\left[\begin{array}{llllll}
\frac{1}{X\left(I_{i}^{1}, J_{i}^{1}\right)} & \cdots & \frac{1}{X\left(I_{i}^{M}, J_{i}^{M}\right)}-\frac{1}{X\left(I_{i}^{1}, J_{i}^{1}\right)} & \cdots & -\frac{1}{X\left(I_{i}^{M}, J_{i}^{M}\right)}
\end{array}\right]\left[\begin{array}{l}
\delta\left(I_{i}^{1}\right) \\
\vdots \\
\delta\left(I_{i}^{M_{i}}\right) \\
\delta\left(J_{i}^{1}\right) \\
\vdots \\
\delta\left(J_{i}^{M_{i}}\right)
\end{array}\right]=g_{i}^{2} \theta_{i}
\end{gathered}
$$

By substituting either (C.7) or (C.8) for $P_{i}$ into (C.2), we get

$$
N_{1} g_{1} \theta_{1}+\cdots+N_{i} g_{i} \theta_{i}+\cdots N_{n} g_{n} \theta_{n} \leq L_{d}
$$

where $g_{i}$ can be either $g_{i}^{1}$ or $g_{i}^{2}$ and $N_{i}=\left[\begin{array}{llll}n_{p, 1 i} & n_{p, 2 i} & \cdots & n_{p, s i}\end{array}\right]^{T}$.

(C.9) can be written in the matrix form

$$
N_{\theta} \theta \leq L_{d}
$$

\footnotetext{
${ }^{1}$ DC power flow solution, referred to "Power system stability and control", P. Kundur, McGraw-Hill Professional
} 
Therefore, the hyperplanes are transformed into another frequently proposed form of the wide-area nomogram consists of inequalities applied to the angle differences measured at different locations. This form is attractive because the phasor angle can be collected in real time from PMU's and be applied directly to assess the risk of cascading contingencies.

Numerical simulation has been conducted to evaluate accuracy of approximation in (C.7) and (C.8) for the same system and base case as in Section 3. It consists of the following two steps.

1) A hyperplane in the tie-line power flow space is created. The coefficients are shown in Table C-1.

Table C- 1. Coefficients of A Hyperplane in the Tie-line Power Flow Space

\begin{tabular}{llllcllll}
\hline $\begin{array}{l}d_{1} \\
\left(n_{1}\right)\end{array}$ & $d_{2}$ & $d_{3}$ & $d_{4}$ & $d_{5}$ & $d_{6}$ & $d_{7}$ & $d_{8}$ & $\mathrm{~L}$ \\
\hline-0.07 & 0.05 & -0.48 & 0.69 & 0.31 & 0.10 & -0.13 & -0.39 & 5047 \\
& & & & & & & & \\
\hline
\end{tabular}

2) Using (C.9), this hyperplane can be transformed into the angle space.

The transformation proposed in (C.9) leads to inaccuracy with the hyperplane in the angle space, which can be quantified by the relative error at a boundary point. The relative error is defined as

$$
E_{r}=\frac{N_{1} g_{1} \theta_{1}+\cdots+N_{i} g_{i} \theta_{i}+\cdots N_{n} g_{n} \theta_{n}-L_{d}}{L_{d}}
$$

where $\theta_{i}$ is the vector of bus angles at both ends of the $i^{\text {th }}$ interface at the boundary point. The distribution of relative errors at 15 boundary points (which were used originally to build the hyperplane in the tie-line power flow space) are shown in Figures C.2 and C.3. The comparison shows that the approximation in (C.5) can result into an error as large as $-8.8 \%$. On the other hand, (C.6) gives more accurate results when transforming the hyperplane in the power flow space into the angle space. This simulation suggests that the voltage magnitude information is necessary to maintain accuracy in the transformation. 


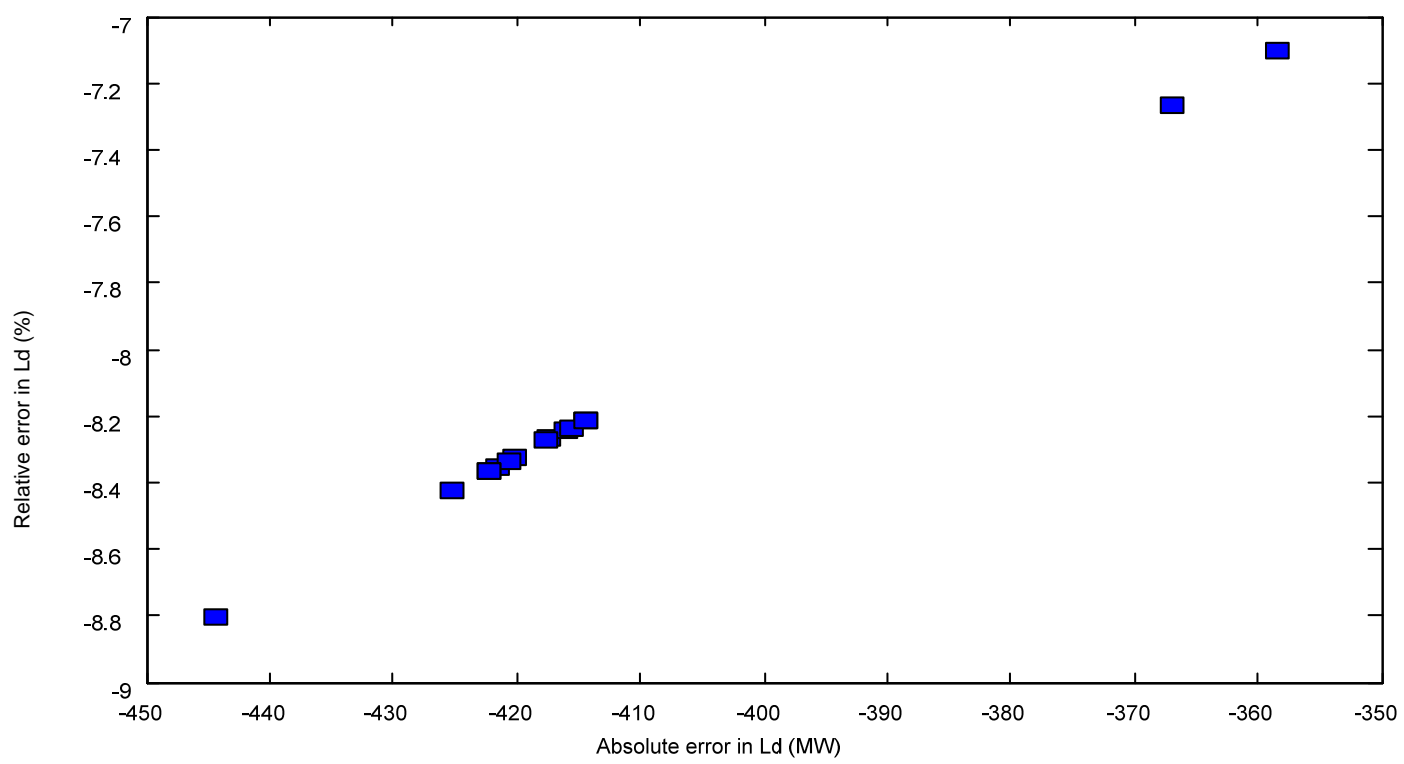

Figure C. 2. Distribution of Relative Errors Caused by Approximation in (C.5)

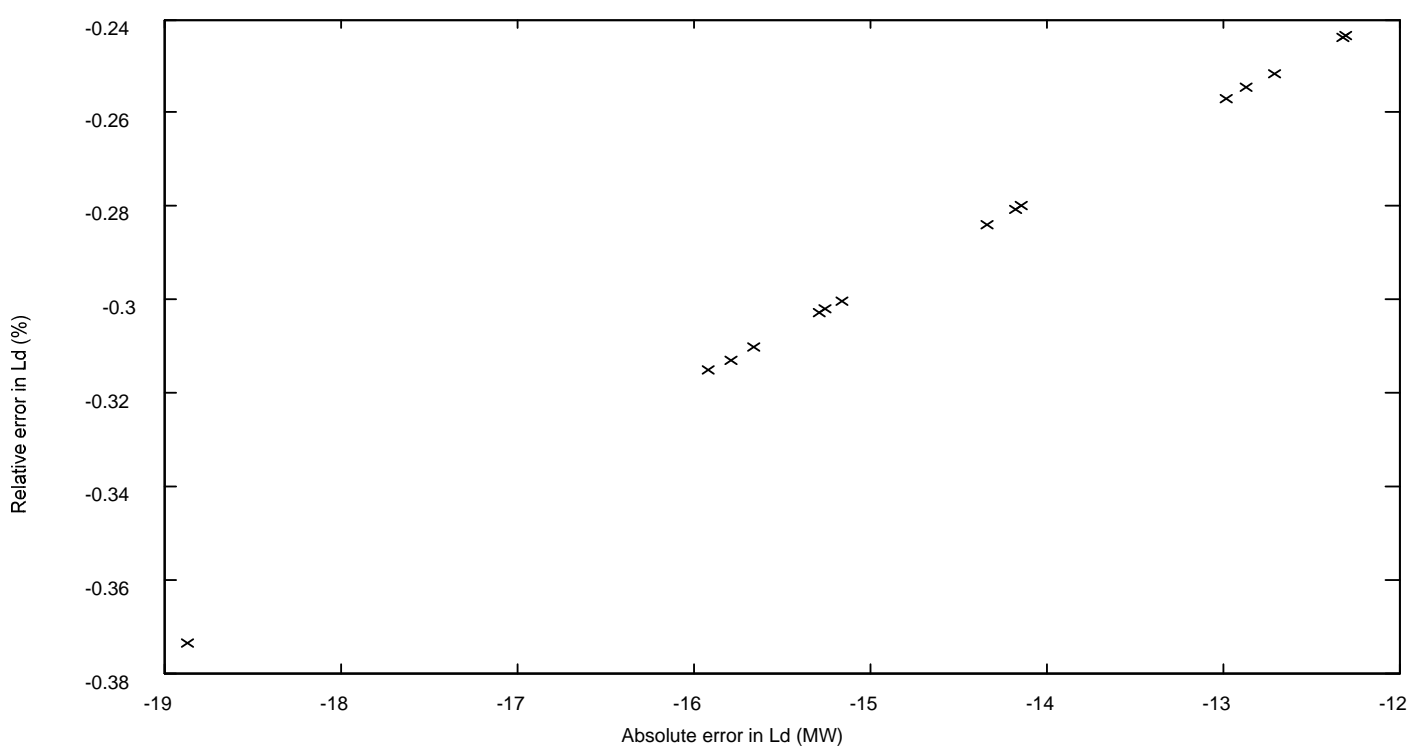

Figure C. 3. Distribution of Relative Errors Caused by Approximation in (C.6) 


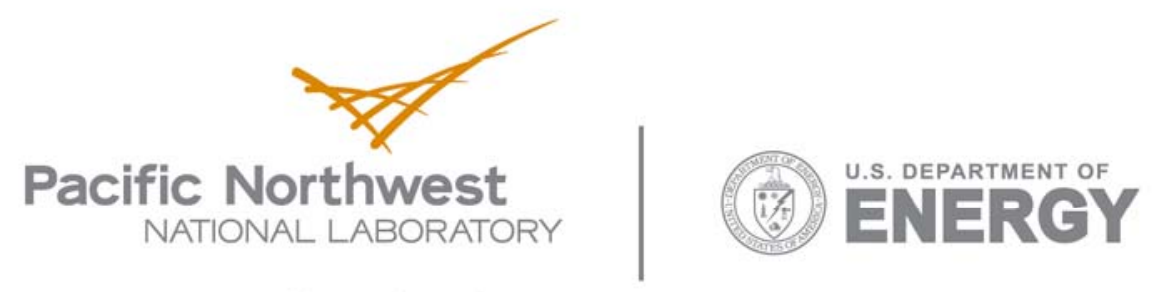

902 Battelle Boulevard

P.O. Box 999

Richland, WA 99352

1-888-375-PNNL (7665)

www.pnl.gov 